\title{
Advanced analytic treatment and efficient computation of the diffraction integrals in the extended Nijboer-Zernike theory
}

\section{S. van Haver}

svenvanhaver@gmail.com

\section{A. J. E. M. Janssen \\ a.j.e.m.janssen@tue.nl}

\begin{abstract}
S[\&]T Experts Pool (STEP), P.O. Box 608, 2600 AP Delft, The Netherlands Optics Research Group, Faculty of Applied Sciences, Technical University Delft, Van der Waalsweg 8, 2628 CH Delft, The Netherlands

Department of Mathematics and Computer Science, Eindhoven University of Technology, P. O. Box 513, 5600 MB Eindhoven, The Netherlands
\end{abstract}

The computational methods for the diffraction integrals that occur in the Extended Nijboer-Zernike (ENZ-) approach to circular, aberrated, defocused optical systems are reviewed and updated. In the ENZ-approach, the Debye approximation of Rayleigh's integral for the through-focus, complex, point-spread function is evaluated in semi-analytic form. To this end, the generalized pupil function, comprising phase aberrations as well as amplitude non-uniformities, is assumed to be expanded into a series of Zernike circle polynomials, and the contribution of each of these Zernike terms to the diffraction integral is expressed in the form of a rapidly converging series (containing power functions and/or Bessel functions of various kinds). The procedure of expressing the through-focus point-spread function in terms of Zernike expansion coefficients of the pupil function can be reversed and has led to the ENZ-method of retrieval of pupil functions from measured through-focus (intensity) point-spread functions. The review and update concern the computation for systems ranging from as basic as having low NA and small defocus parameter to high-NA systems, with vector fields and polarization, meant for imaging of extended objects into a multi-layered focal region.

In the period 2002-2010, the evolution of the form of the diffraction integral ( $\mathrm{DI}$ ) was dictated by the agenda of the ENZ-team in which a next instance of the DI was handled by amending the computation scheme of the previous one. This has resulted into a variety of ad hoc measures, lack of transparency of the schemes, and sometimes prohibitively slow computer codes. It is the aim of the present paper to reconstruct the whole building of computation methods, using consistently more advanced mathematical tools. These tools are

- explicit Zernike expansion of the focal factor in the DI,

- Clebsch-Gordan coefficients for the omnipresent problem of linearizing products of Zernike circle polynomials,

- recursions for Bessel functions, binomials and for the coefficients of algebraic functions that occur as pre-factors of the focal factor in the DI.

This results in a series representation of the DI involving (spherical) Bessel functions and Clebsch-Gordan coefficients, in which the dependence of the DI on parameters of the optical configuration, on focal values, on spatial variables in the image planes, and on degree and azimuthal order of the circle polynomials are separated. This separation of dependencies, together with bounds on Clebsch-Cordan coefficients and spherical Bessel functions, facilitate the error analysis for the truncation of series, showing that in the new scheme the DI can be computed virtually without loss-of-digits. Furthermore, this separation allows for a modular implementation of the computation scheme that offers speed and flexibility when varying the various parameters and variables. The resulting scheme is pre-eminently appropriate for use in advanced optical simulations, where large defocus values, high NA and Zernike terms of high order and degree occur. [DOI: http://dx.doi.org/10.2971/jeos.2013.13044]

Keywords: Diffraction integral, point-spread function, ENZ, semi-analytic computation, vectorial theory, extended object, multilayer

\section{INTRODUCTION AND OVERVIEW}

\section{1 Nijboer-Zernike theory and its extension}

The Nijboer-Zernike (NZ-) theory of diffraction integrals was intended to produce an analytic result that, with the computational means of that time (1930-1950), led to a good approximation of the (intensity) point-spread function in or close to the focal plane of a low-NA, circularly symmetric, aberrated optical system. In this theory, the wave-front aberrations are limited to a few radians, and the amplitude is assumed to be constant on the exit pupil. In the NZ-theory, the aberration phase is expanded on the exit pupil as a linear combi- nation of the Zernike circle polynomials with small and real coefficients, the exponential comprising the aberration phase is linearized, and the resulting integral is evaluated term-byterm per circle polynomial using the basic integral result of the NZ-theory in terms of Jinc functions. For small values of the defocus parameter, the focal factor can be included in the aberration phase (for somewhat larger values, Nijboer uses in his thesis Bauer's formula and an ad hoc procedure for writing products of low-order/degree circle polynomials as sums of circle polynomials after which the basic NZ-result can be used). We refer the reader to Zernike's pioneering 1934 pa- 
per [1], to Nijboer's 1942 thesis [2] on the diffraction theory of aberrations, and to [3], Chap. 9, in particular Secs. 9.1-9.4, and Appendix VII for the early origins of the NZ-theory and for a survey of it, including mathematical properties of the circle polynomials, in a textbook setting.

For the practical problems one encounters nowadays in fields like lithography, microscopy and astronomy, the assumptions of small focus variation, small phase aberrations with constant amplitudes, and low numerical aperture NA are too restrictive, and one has instead resorted to numerical integration or Fourier transform methods to compute the diffraction integrals. The situation changed in 2002 with the appearance of Janssen's paper [4] in which the point-spread function computation method from the classical NZ-theory was extended, allowing one to consider the cases of large defocus value, large phase aberrations and non-uniform amplitudes in the exit pupil. The nature of the basic result in the NZ-theory was changed in this effort [4] from analytic into semi-analytic. Thus, the contributions of the separate circle polynomials (representing the generalized pupil function) to the point-spread function in the focal region are expressed in the form of a rapidly convergent series with terms comprising products of power functions and Jinc functions. We refer to [5] for an assessment of the perspectives of this extended Nijboer-Zernike (ENZ-) approach. For instance, [5] contains an easy-to-use truncation rule for the infinite series in the ENZ-approach, making point-spread function computation feasible in a focal range of some 20 focal depths around the best focus plane and virtual no limitations in the off-axis directions.

The papers [4, 5] were soon followed by a contribution [6] at the 2002 SPIE Microlithography Conference on the problem of the retrieval of aberrations of a circular optical system from measured intensity data in the focal region. In [6], and in [7], the proof-of-principle of the aberration retrieval method is given for the case of small phase-aberrations with uniform amplitude in a practical lithographic setting. As in NZ-theory, the exponential comprising the aberration phase is linearized. Next, the intensity point-spread function (being the squared-modulus of the complex point-spread function) is thought to be linearized around the squared-modulus of the aberration-free, complex point-spread function. By expanding the aberration phase into circle polynomials and applying the semi-analytic result from the ENZ-theory, one thus obtains a (linearized) theoretical expression for the intensity that comprises the unknown expansion coefficients. Then by matching this theoretical expression with the measured intensity data in the focal region, one obtains an estimate of the aberration phase in terms of its Zernike expansion coefficients. The assumption of small aberrations and uniform amplitudes in the ENZ-retrieval method was relaxed in [8] where the full, rather than the exponential part of the pupil function is expanded into circle polynomials. The linearization of the intensity point-spread function should now be amended by an iterative prediction-correction approach in which at each step the deleted small-small cross-terms are estimated by using the previous estimate of the expansion coefficients. The work on ENZ-aberration retrieval has attracted the attention of other workers in various fields, see [9]-[11]; we may also refer to the ENZ-website www.nijboerzernike.nl in which the ENZ-approach for forward computation and aberration retrieval is amply discussed and illustrated with applications.

The ENZ-approach initially assumed low-NA optical systems. Then the focal phase function is accurately approximated as a quadratic function of the distance $\rho$ to the center of the pupil disk of an arbitrary point on the exit pupil. Furthermore, a scalar treatment of the diffraction problem is sufficient so that no vector fields or states of polarization need to be considered. In [12] the ENZ-approach is continued to cover the case of aberrated, high-numerical aperture systems. Using the vectorial framework of Ignatowsky [13] and Richards and Wolf [14], this implies that a total of five basic integrals appear. These basic integrals comprise the high-NA focal factor and account for the radiometric effect while they accommodate the three components of the electric field. For notational convenience a switch is made in [12] from cosine (and sine) form to exponential form of the azimuthal dependence of the circle polynomials. This vectorial version of the ENZ-approach has been used in the formulation and solution of the problem of aberration and birefringence retrieval in [15]. In [16] this high-NA ENZ-retrieval approach is further worked out and simplified, allowing larger aberrations, and extensively illustrated.

The development of the ENZ-approach has been continued in [17] where, based on [18], the ENZ-vector formalism is used for the computation of aerial images of extended objects. This yields the same five basic diffraction integrals as in the vectorial case, except that the finite distance of the object to the entrance pupil has to be accounted for by including a magnification factor in the algebraic pre-factor of the focal factor.

The ENZ-vector formalism for extended objects has been further generalized in [19] where a multilayer structure is assumed in the region around the image plane. The diffraction integrals should now account for both forward and backward propagation in a particular layer of the stack, and this brings about further complications in the algebraic pre-factor of the focal factor. A detailed study of the ENZ-approach for forward calculation and retrieval, going all the way from the basic system with low NA and small defocus to the high-NA system imaging extended objects in a multilayer structure in the focal region is given in [20]. In [20] the complete optical diffraction integrals are presented as well as the final basic integrals (occurring after inserting Zernike expansions) that remain to be computed. An account of the developments of the diffraction integral and its ENZ-computation until 2007 (high NA, vectorial) can be found in [21]. In [21] one can also find the whole history of the optical point-spread function from the $19^{\text {th }}$ century onwards, including an extensive discussion on approximation of Rayleigh's diffraction integral by Debye's integral.

\subsection{Scope and main achievements}

The present paper focuses on the computation of optical diffraction integrals, arising in the form of Debye's approximation of Rayleigh's diffraction integral, for which the approach of the classical Nijboer-Zernike theory is followed. In this approach, the generalized pupil function, vanishing out- 
side the circular pupil disk, is thought to be expanded as a series involving the orthogonal Zernike circle polynomials. Each of these circle polynomials contribute, by linear superposition, to the diffraction integral, yielding basic diffraction integrals per term. The optical systems considered range from as basic as having low NA and small defocus parameter all the way to high-NA systems with vector fields and polarization, meant for imaging of extended objects into a multilayer structure. The main achievement of this paper is the presentation of a computation scheme that systematically serves this whole range of optical situations, using more advanced mathematical tools, thereby replacing the ad hoc procedures and less efficient and less accurate approaches that were presented earlier in the ENZ-domain. In this new computation scheme, the diffraction integrals per circle polynomial are expressed as infinite series in which the dependence of the terms on parameters of the optical system, the defocus parameter, the spatial variables in the image plane, and the degree and azimuthal order of the involved circle polynomials are separated. The use of more advanced mathematical tools yield series for these basic ENZ-diffraction integrals for which the truncation error analysis is transparent, showing rapid convergence with hardly any loss-of-digits. Furthermore, due to separation of dependencies, a modular implementation of the method is feasible, offering flexibility and speed when varying the values of the various parameters and variables. Therefore, this new computation method can serve pre-eminently as a basis for developing simulation tools in advanced optical engineering, virtually without limitations on the required accuracy.

The new method is to be compared to what DFT-methods offer for computation of diffraction integrals in the optical engineering context. In this DFT-practice, it is used that the Debye integral has the form of a 2D Fourier integral that can be discretized using a square grid that covers the pupil disk on which the generalized pupil function is located. This discretized Fourier transform has a fast implementation (2D FFT) in which the values of the diffraction integral can be approximated in a particular image plane at all points on a corresponding square grid in that image plane with a computational load that grows only as $S \ln S$ in the numbers $S$ of grid points used. The DFT-based method does not require a decomposition of the generalized pupil function into circle polynomials.

The accuracy of the computed values in the DFT-method at the grid points in the image planes is limited by the intrinsic discontinuity of the pupil function at the rim of the disk. Furthermore, by varying the defocus parameter, the DFTintegrand can show rapid spatial oscillations on the pupil disk, necessitating to increase the sample frequencies in accordance with, what is vaguely termed as, the Nyquist criterion. Despite all this, for many applications in optical engineering, with requirements like $10^{-4}$ absolute accuracy, the use of DFTmethods is quite practicable. However, in advanced optical simulations, such as those required for the performance assessment of modern lithography tools, the required accuracy and the desired flexibility to vary optical parameters and variables are such that the new ENZ-computation scheme is in a better position. In this advanced practice, the degree $n$ and order $m$ of the involved circle polynomials can range to values up to $n=100$ and $m=20$, which is to be compared to maximum values $n=12$ and $m=6$ in more conventional settings.

The ENZ-method does require decomposition of the generalized pupil function into circle polynomials. In [22], Subsection 2.4, there is proposed a cosine-sampling-and-fitting approach to estimate Zernike expansion coefficients that can handle quite complicated generalized pupil functions in a limited amount of time. In [22], the azimuthal orders and degree of the required circle polynomials can get as high as $|m|=12$ and $n=100$.

\subsection{Organization of this paper}

This paper is organized as follows. In Subsection 2.1 we give a detailed account of the classical NZ-theory, with emphasis on the basic integral result of this theory (to be used after Zernike expansion of the aberration phase and linearization of the exponential comprising this phase). The semi-analytic extension of this basic integral result, in the context of generalized pupil functions, is given in Subsection 2.2. In Section 3 we describe the evolution of the basic integral in the ENZ-theory from the most basic optical situation with low NA and small defocus to the most complicated, vectorial case with extended objects being imaged in a multilayer structure. In Section 4, which is at the heart of the matter as to computation of basic ENZintegrals, the general plan is explained by identifying the substages that are common to computation of all basic integrals. These sub-stages include Zernike expansion of focal factors, Zernike expansion of certain algebraic functions that occur as pre-factors, and linearization of products of radial polynomials, with the ultimate goal that one reaches a position where the basic NZ-integral result can be used in one or another form. In Sections 5-9 the details for the various instances of the basic ENZ-integrals are presented. In Section5 on the simplest situation of low NA and small defocus, Bauer's formula is used and the details about linearization of products of radial polynomials are presented as a key step in the computation. In Section 6 the result of Section 5 is extended to the case of low NA but large defocus, necessitating use and Zernike expansion of the focal factor comprising the correct focal phase rather than its quadratic approximation. In Section 7 systems with high NA are considered, which implies that vector field considerations are needed now and that the radiometric effect must be taken care of. In Section 8 the case of high-NA systems with extended object at finite distance is considered; this comes at the expense of a modest complication in the form of the algebraic pre-factor. Finally, in Section 9, the case of a highNA system imaging extended objects in a multilayer structure near the image plane is considered. Now, the algebraic prefactor is not only further complicated, but also its nature for the case of backward propagation is so different compared to the case of forward propagation that an adaptation of the basic NZ-integral is needed. In Section 10 we present comments on modular implementation and computation time gains, and we illustrate the convergence behaviour of the series representation of the basic ENZ-integrals. In Section 11 we present our conclusions. Finally, in the 8 appendices we present the mathematical derivations of results used in the main text in the interest of a smooth development of the core material. 


\section{THE BASIC INTEGRAL RESULT IN THE NIJBOER-ZERNIKE THEORY AND ITS EXTENSION TO LARGE DEFOCUS VALUES}

In this section we present a concise sketch of the classical Nijboer-Zernike theory, aimed at complex amplitude computation for low-NA circular optical systems with small purephase aberrations. After linearizing the exponential containing the aberration phase and expanding this aberration phase into the orthogonal circle polynomials of Zernike, there arise integrals involving the low-NA focal factor $\exp \left[\right.$ if $\left.\rho^{2}\right]$, a Bessel function and the radial part of a Zernike circle polynomial. The order of the Bessel function equals the azimuthal order of the radial polynomial. In case $f=0$, these integrals can be evaluated in closed form, and this constitutes the basic result in the classical NZ-theory. A semi-analytic extension of this result has been obtained by Janssen [4]. In Subsec. 2.1 we present the sketch of the NZ-theory and in Subsec. 2.2 we give its semi-analytic extension.

\subsection{Classical Nijboer-Zernike theory}

We consider a point source of monochromatic light in the object plane of a centered, circular optical system, see Figure 1, and we choose coordinates

$$
(\nu, \mu)=(\rho \cos \vartheta, \rho \sin \vartheta) \text { and }(x, y)=(r \cos \varphi, r \sin \varphi)
$$

with $\rho \geq 0,0 \leq \vartheta<2 \pi$ and $r \geq 0,0 \leq \varphi<2 \pi$ in the exit pupil and image plane, respectively. We assume that the optical system has a circular pupil of radius 1 with center at $(v, \mu)=0(\rho=0)$. The optical system is assumed to be aberrated and the aberration is described by a pupil function $P$ that we write in the form

$$
\begin{gathered}
P(\rho, \vartheta)=A(\rho, \vartheta) \exp [i \Phi(\rho, \vartheta)], \\
0 \leq \rho \leq 1, \quad 0 \leq \vartheta<2 \pi,
\end{gathered}
$$

where $A$ is the (non-negative) transmission function and $\Phi$ is the (real) aberration phase. When the system is aberrationfree we have $A \equiv 1, \Phi \equiv 0$ on the pupil. The pupil function in Eq. (2) is a generalized pupil function since we allow nonconstant transmission functions. Zernike in [1], Nijboer in [2] and Born and Wolf in [3] consider the case that $A \equiv 1$ on the pupil, and in [2], [3] the attention is limited to the case that $\Phi(\rho, \vartheta)$ is an even function of $\vartheta: \Phi(\rho, 2 \pi-\vartheta)=\Phi(\rho, \vartheta)$, $0 \leq \vartheta<2 \pi$.

The normalized complex point-spread function $U(x=r \cos \varphi, y=r \sin \varphi ; f)$ at a defocused plane described by the defocus parameter $f$ in image space is given according to Fourier optics as

$$
\begin{aligned}
& U(r, \varphi ; f)= \\
& \frac{1}{\pi} \int_{0}^{1} \int_{0}^{2 \pi} \exp \left[i f \rho^{2}\right] P(\rho, \vartheta) \exp [2 \pi i \rho r \cos (\vartheta-\varphi)] \rho d \rho d \vartheta,
\end{aligned}
$$

where we have used, see Eq. (1), that

$$
\exp [2 \pi i v x+2 \pi i \mu y]=\exp [2 \pi i \rho r \cos (\vartheta-\varphi)] .
$$

The exponential in Eq. (4) is dictated by the Huygens-Fresnel principle, and the focal factor $\exp \left[i f \rho^{2}\right]$ is valid under the assumption that the system has low-to-medium numerical aperture NA (say, NA $\leq 0.6$ ). The integral in Eq. (3) is the Debye approximation of the Rayleigh integral for the point-spread function, see [3], § 8.8 and [21], Subsecs. 2.1-4.

To proceed to the computation of $U$ in Eq. (3), the assumptions made in [1], [2], [3] on $P$ in Eq. (2) are that $A \equiv 1$ and that $\Phi$ is relatively small, say $|\Phi| \leq 1 / 2$. The exponential $\exp [i \Phi]$ can then be linearized as $1+i \Phi$ at the expense of a small error. The next step in the NZ-theory is to expand $\Phi$ into a Zernike series,

$$
\begin{gathered}
\Phi(\rho, \vartheta)=\sum_{n, m}\left(\alpha_{n, c}^{m} \cos m \vartheta+\alpha_{n, s}^{m} \sin m \vartheta\right) R_{n}^{m}(\rho), \\
0 \leq \rho \leq 1, \quad 0 \leq \vartheta<2 \pi .
\end{gathered}
$$

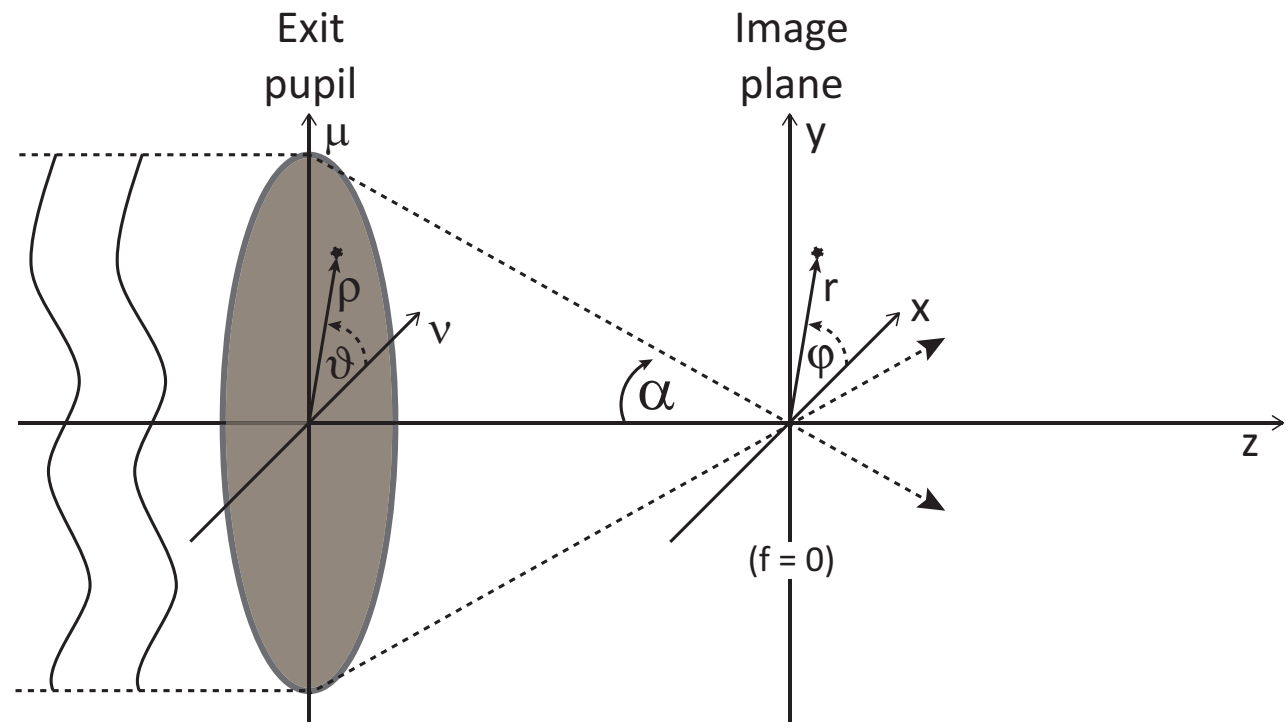

FIG. 1 The basic optical configuration considered in the ENZ-theory. A point-source at infinity generates an incident plane-wave that is captured by the optical system and results into a converging wave-front in the exit pupil towards the image plane. The position on the exit pupil is defined by the cylindrical coordinates ( $\rho, \vartheta)$; the position in the image plane region is defined by the cylindrical coordinate system $(r, \phi, f)$. The maximum aperture (NA) of the imaging pencil is defined as $s_{0}=\sin \alpha_{m a x}$. 
In Eq. (5) the summation is over all non-negative integer $n$ and $m$ such that $n-m$ is even and non-negative, and all $\alpha^{\prime}$ s are real. The functions $R_{n}^{m}(\rho) \cos m \vartheta, R_{n}^{m}(\rho) \sin m \vartheta$ constitute an orthogonal base for the set of functions vanishing outside the unit disk and are called Zernike circle polynomials. The radial part $R_{n}^{m}(\rho)$ of a circle polynomial is a polynomial in $\rho$ of degree $n$ and azimuthal order $m$ and has non-zero coefficients for the powers $\rho^{m}, \rho^{m+2}, \ldots, \rho^{n}$ only. Accordingly, $R_{0}^{0}(\rho)=1$, and it is customary to assume that $\alpha_{0, c}^{0}=\alpha_{0, s}^{0}=0$. For an extensive review of the Zernike circle polynomials in the context of the NZ-theory, see [3], Chap. 9 and Appendix VII.

After using the result, see [14], (2.9)

$$
\begin{aligned}
& \int_{0}^{2 \pi} \exp [2 \pi i \rho r \cos (\vartheta-\varphi)]\left\{\begin{array}{l}
\cos m \vartheta \\
\sin m \vartheta
\end{array} d \vartheta\right. \\
&=2 \pi i^{m} J_{m}(2 \pi \rho r) \quad\left\{\begin{array}{l}
\cos m \varphi \\
\sin m \varphi
\end{array},\right.
\end{aligned}
$$

with $J_{m}$ the Bessel function of the first kind and of order $m$, and approximating $P$ as $1+i \Phi$ in Eq. (3), there results

$$
\begin{aligned}
& U(r, \varphi ; f)=2 V_{0}^{0}(r, f) \\
& \quad+2 i \sum_{n, m} i^{m}\left(\alpha_{n, c}^{m} \cos m \varphi+\alpha_{n, s}^{m} \sin m \varphi\right) V_{n}^{m}(r, f),
\end{aligned}
$$

in which

$$
V_{n}^{m}(r, f)=\int_{0}^{1} \exp \left[i f \rho^{2}\right] R_{n}^{m}(\rho) J_{m}(2 \pi r \rho) \rho d \rho
$$

The constant term 1 in the approximation $1+i \Phi$ of $P$ yields the $2 V_{0}^{0}$ in Eq. (7) and is under the assumptions made dominant. The basic integral result of the NZ-theory is that $V_{n}^{m}(r, f=0)$ can be evaluated explicitly as

$$
\begin{gathered}
V_{n}^{m}(r, 0)=\int_{0}^{1} R_{n}^{m}(\rho) J_{m}(2 \pi r \rho) \rho d \rho= \\
(-1)^{\frac{n-m}{2}} \frac{J_{n+1}(2 \pi r)}{2 \pi r} .
\end{gathered}
$$

A proof of Eq. (9) is given by Zernike in [1] using the WeberSchafheitlin discontinuous integral, see [23], 10.22 .56 (case $b=1, a=\rho<1, \lambda=0, v=n+1, \mu=m$ ), together with the inversion formula for Hankel transforms. Another proof of Eq. (9) is given bij Nijboer in [2]. This proof uses the connection $R_{n}^{m}(\rho)=\rho^{m} P_{p}^{(0, m)}\left(2 \rho^{2}-1\right), p=\frac{1}{2}(n-m)$, between the radial part of the circle polynomials and the Jacobi polynomials $P_{k}^{(\alpha, \beta)}(x)$, see [23], Chap. 18, together with the power series expansion of the Bessel functions and Rodriguez' formula for the Jacobi polynomials to evaluate the integrals $\int_{0}^{1} \rho^{m+2 k} R_{n}^{m}(\rho) \rho d \rho$. Nijboer's argument is presented very concisely in [2], p. 25; it is expanded in [3], Appendix VII.

Thus Eq. (7) together with Eq. (9) give an analytic result for the point-spread function $U(r, \varphi ; f=0)$ in terms of the expansion coefficients $\alpha$ in Eq. (5) and the Jinc functions $J_{n+1}(2 \pi r) / 2 \pi r$. For the case that $f \neq 0$ but small, Nijboer in
[2] proceeds by using Bauer's formula (see [23], 10.60 .7 with $\cos \alpha=2 \rho^{2}-1$ and observing that $\left.R_{2 k}^{0}(\rho)=P_{k}\left(2 \rho^{2}-1\right)\right)$

$$
\exp \left[i f \rho^{2}\right]=\exp \left[\frac{1}{2} i f\right] \sum_{k=0}^{\infty}(2 k+1) i^{k} j_{k}\left(\frac{1}{2} f\right) R_{2 k}^{0}(\rho),
$$

with $j_{k}$ the spherical Bessel function, see [23], 10.47, of order $k$. Assuming small $f$ and only a few significant terms in Eq. (5), Nijboer then wrote a limited number of products $R_{2 k}^{0}(\rho) R_{n}^{m}(\rho)$, that appear in the integral in Eq. (8) when Eq. (10) is used, in an ad hoc fashion as a finite linear combination of radial polynomials of azimuthal order $m$. To each of the terms in this linear combination, the basic result in Eq. (9) can be used and Nijboer arrived at an approximate expression for the point-spread function $U$. In all this it should be noted that computational means of the sort we have nowadays were not available at that time.

In the present paper we work with generalized pupil functions $P$ as in Eq. (2), with non-uniform $A$ and real but possibly large phase aberrations $\Phi$. Moreover, we take as definition of the Zernike circle polynomials the form with exponential, rather than trigonometric, azimuthal dependence. Thus, a generalized pupil function $P$ is expanded as

$$
\begin{gathered}
P(\rho, \vartheta)=A(\rho, \vartheta) \exp [i \Phi(\rho, \vartheta)]=\sum_{n, m} \beta_{n}^{m} Z_{n}^{m}(\rho, \vartheta), \\
0 \leq \rho \leq 1,0 \leq \vartheta<2 \pi,
\end{gathered}
$$

in which

$$
\begin{gathered}
Z_{n}^{m}(\rho, \vartheta)=R_{n}^{|m|}(\rho) \exp [i m \vartheta] \\
0 \leq \rho \leq 1, \quad 0 \leq \vartheta<2 \pi,
\end{gathered}
$$

and where $n$ and $m$ in the summation in Eq. (11) and in Eq. (12) are integers such that $n-|m|$ is even and non-negative. We refer to [24] where, apparently, generalized pupil functions were expanded as in Eq. (11) for the first time, for the purpose of computation of the optical transfer function. With these conventions, the basic result Eq. (9) takes the form

$$
\begin{aligned}
& \iint_{v^{2}+\mu^{2} \leq 1} \exp [2 \pi i v x+2 \pi i \mu y] Z_{n}^{m}(\nu, \mu) d v d \mu= \\
& \int_{0}^{1} \int_{0}^{2 \pi} \exp [2 \pi i \rho r \cos (\vartheta-\varphi)] R_{n}^{|m|}(\rho) \exp [i m \vartheta] \rho d \rho d \vartheta= \\
& 2 \pi i^{n} \frac{J_{n+1}(2 \pi r)}{2 \pi r} \exp [i m \varphi],
\end{aligned}
$$

where Eq. (6) and Eq. (9) have been used. Equivalently, using $J_{m}(z)=i^{m-|m|} J_{|m|}(z)$, we have

$$
\int_{0}^{1} R_{n}^{|m|}(\rho) J_{m}(2 \pi r \rho) \rho d \rho=(-1)^{\frac{n-m}{2}} \frac{J_{n+1}(2 \pi r)}{2 \pi r} .
$$

\subsection{Semi-analytic extension of the basic NZ-integral result to defocused systems}

The result Eq. (9) of the NZ-theory has been extended by Janssen in [4] in 2002 to cover the case of general $f \neq 0$ in 
Eq. (8). With $n$ and $m$ non-negative integers such that $n-m$ is even and non-negative, we let

$$
p=\frac{1}{2}(n-m), \quad q=\frac{1}{2}(n+m) .
$$

Then it holds that

$$
\begin{gathered}
V_{n}^{m}(r, f)=\int_{0}^{1} \exp \left[i f \rho^{2}\right] R_{n}^{m}(\rho) J_{m}(2 \pi r \rho) \rho d \rho= \\
\exp [i f] \sum_{l=1}^{\infty}(-2 i f)^{l-1} \sum_{j=0}^{p} v_{l j} \frac{J_{m+l+2 j}(2 \pi r)}{l(2 \pi r)^{l}}
\end{gathered}
$$

in which

$$
\begin{array}{r}
v_{l j}=(-1)^{p}(m+l+2 j)\left(\begin{array}{c}
m+j+l-1 \\
l-1
\end{array}\right) . \\
\left(\begin{array}{c}
j+l-1 \\
l-1
\end{array}\right)\left(\begin{array}{l}
l-1 \\
p-j
\end{array}\right) /\left(\begin{array}{c}
q+l+j \\
l
\end{array}\right)
\end{array}
$$

for $j=0,1, \ldots, p$ and $l=1,2, \ldots$. The formula Eq. (16) can be brought into a form reminiscent of Lommel's solution for the case of an aberration-free, defocused system, see [3], Subsec. 8.8.1. In its most compact form Lommel's solution reads

$$
V_{0}^{0}(r, f)=\exp [i f] \sum_{l=0}^{\infty}\left(\frac{-i f}{\pi r}\right)^{l} \frac{J_{l+1}(2 \pi r)}{2 \pi r} .
$$

Letting

$$
\begin{array}{r}
u_{l j}=(-1)^{p} \frac{m+l+2 j+1}{q+l+j+1}\left(\begin{array}{c}
m+l+j \\
l
\end{array}\right) . \\
\left(\begin{array}{c}
j+l \\
l
\end{array}\right)\left(\begin{array}{c}
l \\
p-j
\end{array}\right) /\left(\begin{array}{c}
q+l+j \\
l
\end{array}\right)
\end{array}
$$

for $j=0,1, \ldots, p$ and $l=0,1, \ldots$, we can write Eq. (16) as

$$
V_{n}^{m}(r, f)=\exp [i f] \sum_{l=0}^{\infty}\left(\frac{-i f}{\pi r}\right)^{l} \sum_{j=0}^{p} u_{l j} \frac{J_{m+l+2 j+1}(2 \pi r)}{2 \pi r}
$$

The convergence of the series in Eq. (16) or Eq. (20) is rapid, provided that $f$ is not too large. In [5], Appendix B a rule-ofthumb has been presented for the number of terms $l$ of the infinite series that should be included: taking the first $\lceil 3|f|+5\rceil$ terms in the series gives in all cases an absolute accuracy $10^{-6}$. However, when $|f| \geq 25$, the powers of $f$ in the series cause loss-of-digits to an extent that this $10^{-6}$ accuracy gets out of sight when computing with $10^{-15}$ absolute accuracy.

To remedy the latter situation, there has been developed in [25] a method to compute the $V_{n}^{m}$ in, what is called in [25], a Bessel-Bessel series form (as opposed to the power-Bessel series forms in Eq. (16) and Eq. (20)). This method is based on Nijboer's approach of using Bauer's identity Eq. (10) together with a linearization procedure for writing products $R_{2 k}^{0} R_{n}^{m}$ as linear combination of radial polynomials of azimuthal order $m$. In [26], this approach is elaborated for the case that $n=m=0$ (so that the linearization procedure is a triviality) and yields an alternative way to compute Lommel's solution $V_{0}^{0}$ in Eq. (18). In general, the result is a series representation of the form

$$
V_{n}^{m}(r, f)=\exp \left[\frac{1}{2} i f\right] \sum_{h, k} E_{h k}(m, n) j_{k}\left(\frac{1}{2} f\right) \frac{J_{h+1}(2 \pi r)}{2 \pi r}
$$

with numbers $E$ of modulus 1 or less. Due to the decay properties of (spherical) Bessel functions with increasing order, the number of terms to be used in the double series in Eq. (21) should be somewhat larger than $2 \pi r|f|$, and there is virtually no loss-of-digits. In [25] the numbers $E$ were determined in a somewhat clumsy, though structural, manner, a point about which we improve considerably in the sequel.

\section{EVOLUTION OF THE FORM OF THE BASIC ENZ-INTEGRAL}

In this section we present the integral expressions that occur after the Zernike expansion of the generalized pupil function, containing phase-aberrations and amplitude nonuniformities, has been inserted into the diffraction integral that describe the particular optical situations considered in Sec. 1.

\subsection{Basic low-NA, small-defocus system (B)}

The Debye approximation of the Rayleigh integral for the point-spread function can be approximated further in the case of low-NA systems. The focal factor $\exp \left[\right.$ if $\left.\rho^{2}\right]$ that occurs in Eq. (3) is a low-NA approximation of the true focal factor

$$
\exp \left[i \frac{f}{u_{0}}\left(1-\sqrt{1-s_{0}^{2} \rho^{2}}\right)\right], \quad 0 \leq \rho \leq 1,
$$

in which $s_{0}$ is the numerical aperture (NA) of the optical system with $0<s_{0}<1$, and

$$
u_{0}=1-\sqrt{1-s_{0}^{2}} .
$$

For small values of $s_{0}$, say $s_{0} \leq 0.6$, and small values of $f$, say $|f| \leq \frac{1}{2} \pi$, the approximation $\exp \left[\right.$ if $\left.\rho^{2}\right]$ of the focal quantity in Eq. (22) comes with only a small error, and then the expression for the point-spread function is valid. Inserting the Zernike expansion Eq. (11) of the generalized pupil function $P$ into the integral in Eq. (3) yields the basic integral

$$
I_{\mathrm{B}}=V_{n}^{m}(r, f)=\int_{0}^{1} \exp \left[i f \rho^{2}\right] R_{n}^{|m|}(\rho) J_{m}(2 \pi r \rho) \rho d \rho
$$

with integer $n$ and $m$ such that $n-|m|$ is even and nonnegative. This is the starting point in [4]-[7].

\subsection{Basic low-NA, large-defocus systems (BLD)}

In the case of low NA $s_{0}$ but large defocus value $f$, the quantity if $\rho^{2}$ ceases to be a valid approximation of the quantity $\left(\right.$ if $\left./ u_{0}\right)\left(1-\sqrt{1-s_{0}^{2} \rho^{2}}\right)$ in the exponential in Eq. (22). The diffraction integral for the point-spread function in Eq. (3) should now contain the true focal factor in Eq. (22) instead of $\exp \left[i f \rho^{2}\right]$. Expanding $P$ as in Eq. (11), the basic integral

$$
\begin{aligned}
& I_{\mathrm{BLD}}= \\
& \quad \int_{0}^{1} \exp \left[\frac{i f}{u_{0}}\left(1-\sqrt{1-s_{0}^{2} \rho^{2}}\right)\right] R_{n}^{|m|}(\rho) J_{m}(2 \pi r) \rho d \rho
\end{aligned}
$$


arises.

Along with $I_{\mathrm{BLD}}$ in Eq. (25), we shall consider

$$
\begin{aligned}
& \bar{I}_{\mathrm{BLD}}= \\
& \int_{0}^{1} \frac{\exp \left[\frac{i f}{u_{0}}\left(1-\sqrt{1-s_{0}^{2} \rho^{2}}\right)\right]}{\sqrt{1-s_{0}^{2} \rho^{2}}} R_{n}^{|m|}(\rho) J_{m}(2 \pi r \rho) \rho d \rho .
\end{aligned}
$$

The integrals in Eq. (25) and Eq. (26) occur when in the process of going from the true Rayleigh integral to the basic integral in Eq. (3) a particular approximation is carried through or not. The treatment of the $\bar{I}_{\mathrm{BLD}}$-integral is mathematically slightly more convenient than that of the $I_{\mathrm{BLD}}$-integral. A semi-analytic expression for the $I_{\mathrm{BLD}}$-integral is readily obtained from such an expression for the $\bar{I}_{\mathrm{BLD}}$-integral by differentiation with respect to $f$.

An alternative solution for the BLD-case, that works for moderately large values of $f$, is to approximate

$$
\frac{i f}{u_{0}}\left(1-\left(1-\sqrt{1-s_{0}^{2} \rho^{2}}\right)\right) \text { by } \bar{g}+i \bar{f} \rho^{2}
$$

where $\bar{g}$ and $\bar{f}$ are such that the minimum of

$$
\int_{0}^{1} \mid \frac{i f}{u_{0}}\left(1-\left(1-\sqrt{1-s_{0}^{2} \rho^{2}}\right)-a-\left.i b \rho^{2}\right|^{2} \rho d \rho\right.
$$

is assumed for $a=\bar{g}, b=\bar{f}$. This approach brings us back to the $B$-case in Subsec. 3.1 and allows somewhat larger values of $s_{0}$, say, up to 0.8 . This option has been worked out in detail in [8], Sec. 5, but we shall not consider it further in this paper.

The choice between the two options in Eq. (25) and Eq. (26) may also depend on whether one is concerned about accurate forward computation or about retrieval. In the case of forward computation from the Zernike expansion of $P$, it is better to use $\bar{I}_{\mathrm{BLD}}$ in Eq. (26) since it entails a more accurate approximation of the true Rayleigh integral than $I_{\mathrm{BLD}}$ in Eq. (25) does. In the case of retrieval of $P$ from intensity data in the focal region as described in Sec. 1, it does not matter whether one uses Eq. (25) or Eq. (26). Using Eq. (25) for this purpose, one retrieves an approximation of $P(\rho, \vartheta) / \sqrt{1-s_{0}^{2} \rho^{2}}$ instead of $P(\rho, \vartheta)$ itself, and so one simply has to correct the retrieval result for the factor $\sqrt{1-s_{0}^{2} \rho^{2}}$ afterwards. A similar comment applies in the case of the high-NA systems in Subsec. 3.3 where one may choose to include or not to include a factor $\left(1-s_{0}^{2} \rho^{2}\right)^{-1 / 4}$ in the basic integral.

\subsection{High-NA systems with vector fields (V)}

In [12] the vector field in the focal region of an aberrated highaperture optical system is expressed in terms of the Zernike expansion coefficients of the generalized pupil function. In this case polarization may occur, and this means that one has to consider two Zernike expansions in the pupil, viz. one expansion for the $x$-direction and one expansion for the $y$ direction in image space. The diffraction integrals for the three components of the electric field are derived from the classical Ignatowsky/Richards-and-Wolf formulas, see [13] and
[14]. After inserting the Zernike expansions into the diffraction integrals, one obtains a new set of basic ENZ-integrals, and these are presented in [12], Sec. 4 (forward computation) and [15], Sec. 1.A, and [16], Sec. 2.7. Depending on how one wants to include the radiometric effect (see [12], Eq. (17), and [15], Eq. (4), [16], Eq. (4), [21], Eq. (2.67), respectively), the basic integrals

$$
\begin{aligned}
& I_{\mathrm{V}}=\int_{0}^{1}\left(1+\sqrt{1-s_{0}^{2} \rho^{2}}\right)^{-|j|+1} . \\
& \exp \left[\frac{i f}{u_{0}}\left(1-\sqrt{1-s_{0}^{2} \rho^{2}}\right)\right] \rho^{|j|} R_{n}^{|m|}(\rho) J_{m+j}(2 \pi r \rho) \rho d \rho
\end{aligned}
$$

and

$$
\begin{aligned}
& \bar{I}_{\mathrm{V}}=\int_{0}^{1} \frac{\left(1+\sqrt{1-s_{0}^{2} \rho^{2}}\right)^{-|j|+1}}{\left(1-s_{0}^{2} \rho^{2}\right)^{1 / 4}} \cdot \\
& \exp \left[\frac{i f}{u_{0}}\left(1-\sqrt{1-s_{0}^{2} \rho^{2}}\right)\right] \rho^{|j|} R_{n}^{|m|}(\rho) J_{m+j}(2 \pi r \rho) \rho d \rho
\end{aligned}
$$

arise. In Eqs. (29a)-(29b) we have integer $n$ and $m$ such that $n-|m|$ is even and non-negative as usual, and $j=-2,-1,0,1,2$. The sole difference between Eq. (29a) and Eq. $(29 b)$ is the factor $\left(1-s_{0}^{2} \rho^{2}\right)^{-1 / 4}$ that one can choose to include in the pupil functions (as is done in [15], [16] and [21]) or not (as is done in [12]). In the case of forward computation, more accurate results are obtained by using Eq. (29b), while for the purpose of ENZ-retrieval one can use Eq. (29a) as well when the retrieved function is corrected for the factor $\left(1-s_{0}^{2} \rho^{2}\right)^{-1 / 4}$ afterwards.

\subsection{High-NA systems with vector fields and object at finite distance (VM)}

In [17] vectorial aerial-image ENZ-computations are presented for the imaging of extended objects at finite distance from the entrance pupil of the system (see also Figure 2). The diffraction integrals for the three field components are developed using the approach in [18], and these integrals now contain the refractive indices $n_{0}$ and $n_{1}$ of both the object and image space, see [17], Eqs. (20)-(22). Inserting the Zernike expansions of the two components of the field in the entrance pupil, one arrives at the basic integral

$$
\begin{aligned}
& I_{\mathrm{VM}}=\int_{0}^{1} \frac{\left\{\left(1-s_{0}^{2} \rho^{2}\right)^{1 / 2}+\left(1-s_{0, M}^{2} \rho^{2}\right)^{1 / 2}\right\}^{-|j|+1}}{\left(1-s_{0}^{2} \rho^{2}\right)^{1 / 4}\left(1-s_{0, M}^{2} \rho^{2}\right)^{3 / 4}} . \\
& \exp \left[\frac{i f}{u_{0}}\left(1-\sqrt{1-s_{0}^{2} \rho^{2}}\right)\right] \rho^{|j|} R_{n}^{|m|}(\rho) J_{m+j}(2 \pi r \rho) \rho d \rho,
\end{aligned}
$$

see [17], Eq. (30) and [20], Eq. (3.30). In Eq. (30) the radiometric effect has been treated in the manner of [15], [16] and [21]. The $s_{0, M}^{2}$ in the integral in Eq. (30) is built from the ratio of the refractive indices in the respective spaces and the magnification factor $M$ due to the finite distance of the object to the optical system according to

$$
s_{0, M}^{2}=\frac{n_{1}^{2}}{n_{0}^{2}} M^{2} s_{0}^{2} .
$$




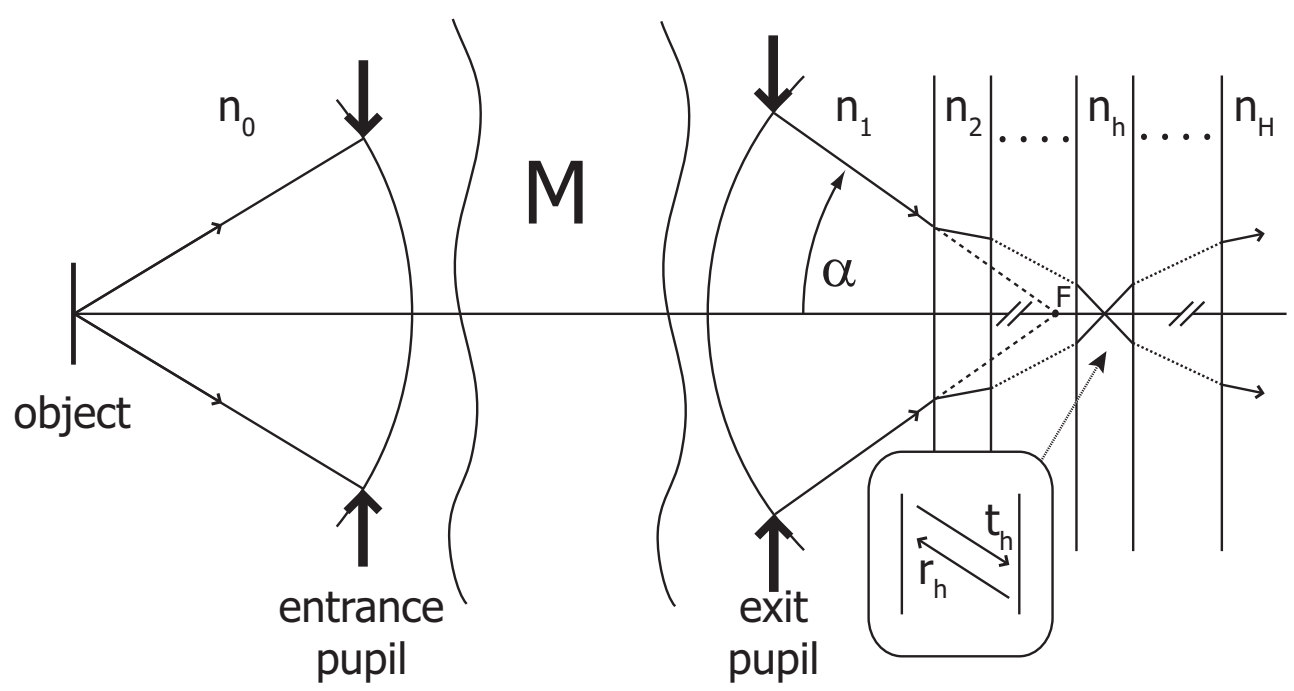

FIG. 2 Schematic representation of imaging of an (extended) object at a finite distance by an optical system with magnification $M$. In the case of a uniform image space, $n_{h}=n_{1}$ for $h=1,2, \cdots, H$, the resulting ENZ-integrals are given in Eq. (30). In the case of a multi-layered image space, the different layers are represented by their effective refractive indices, $n_{h}$, where the subscript refers to the h-th layer starting from the exit pupil. The labels $t_{h}$ and $r_{h}$ pertain to the amplitudes of the forward and backward propagating plane wave components, respectively, which originate from reflections at the layer interfaces. The point $F$ is the geometrical best focus position in case of a uniform image region and the numerical aperture is $\mathrm{NA}=n_{1} \sin \alpha$ with $\sin \alpha=s_{0}$.

\subsection{High-NA systems with vector fields, object at finite distance and multi-layered focal region (VMML)}

In [19] image formation by a high-NA optical system in a multi-layered focal region is considered for objects at finite distance in the ENZ-context (see also Figure 2). The basic assumptions under which [19] proceeds are non-absorbing layers with refractive indices $n_{h}>n_{1} s_{0}$ and absence of nonpropagating waves. The diffraction integrals pertaining to the transmitted and reflected plane wave components in layer $h$ are developed from basic principles in [19], Sec. 3 with [19], Eqs. (19)-(21) as a result. Using Zernike expansions as in the previous cases, this leads to basic integrals

$$
\begin{aligned}
& I_{\mathrm{VMML}}=\int_{0}^{1} \frac{\left\{\left(1-s_{0, h}^{2} \rho^{2}\right)^{1 / 2} \pm\left(1-s_{0, M}^{2} \rho^{2}\right)^{1 / 2}\right\}^{-|j|+1}}{\left(1-s_{0}^{2} \rho^{2}\right)^{1 / 4}\left(1-s_{0, M}^{2} \rho^{2}\right)^{3 / 4}} . \\
& \exp \left[\frac{i f}{u_{0}}\left(1-\sqrt{1-s_{0, h}^{2} \rho^{2}}\right)\right] \rho^{|j|} R_{n}^{|m|}(\rho) J_{m+j}(2 \pi r \rho) \rho d \rho,
\end{aligned}
$$

see [19], Eqs. (35)-(36) and [20], Eq. (3.49). The $s_{0, M}$ accounts for the refractive indices in the object space and in the homogeneous part (before the multilayer) of the image space as well as the magnification due to the finite-distance object as in Eq. (31). The $s_{0, h}$ accounts for the refractive indices $n_{1}$ and $n_{h}$ in the homogeneous part of the image space and layer $h$ in the focal region according to

$$
s_{0, h}^{2}=\frac{n_{1}^{2}}{n_{h}^{2}} s_{0}^{2} .
$$

By the assumption $n_{h}>n_{1} s_{0}$, we have $s_{0, h}^{2}<1$. The + and between the two square roots in the expression between \{\} in the integrand in Eq. (32) refer to the forward and backward propagating plane waves. Due to the assumption of absorption-free media made in [19], no distinction between transmitted and reflected fields needs to be made. In Eq. (32) the radiometric effect has been treated in the manner of [15], [16] and [21], and $j$ and $n, m$ are as earlier in Eqs. (29a), (29b) and (30).

\section{COMPUTATION OF BASIC INTEGRALS: GENERAL OBSERVATIONS}

In this section we present general observations as to the computation of the I-integrals that were presented in Section 3. In the subsequent sections these observations are worked out and detailed for the various optical situations as considered in Subsections 3.1-3.5.

The integrand of the integral expressions $I$ is in all cases a product of

a. a focal factor $\exp \left[\right.$ if $\left.\rho^{2}\right]$ or $\exp \left[\frac{i f}{u}\left(1-\sqrt{1-s^{2} \rho^{2}}\right)\right]$,

b. an algebraic function, containing a combination of functions of the form $\left(1-s^{2} \rho^{2}\right)^{\alpha}$; this function is in all cases, except in the case of $I_{\mathrm{VMML}}$ with $|j|=2$ and back propagating waves, regular on the pupil disk $\rho \leq 1$,

c. a factor $\rho^{|j|} R_{n}^{|m|}(\rho)$,

d. a Bessel function $J_{m+j}(2 \pi r \rho)$.

In $\mathrm{c}$ and $\mathrm{d}$ we have integers $j$ with $|j|=0,1,2$, and $n$ and $m$ are integers with $n-|m|$ even and non-negative.

The general approach is to work towards a situation that we can use the basic result Eq. (14) of the NZ-theory. To that end, the product of the three factors in $a, b$ and $c$ above should be developed as a $R^{|m+j|}$-series, i.e., as a series involving the radial polynomials with azimuthal order $m+j$ equal to the order $m+j$ of the Bessel function in $\mathrm{d}$.

The first step in this process is to use an explicit result for the $R^{0}$-expansion of the focal factor. In case that the focal factor is given by $\exp \left[\right.$ if $\left.\rho^{2}\right]$, there is Bauer's formula

$$
\exp \left[i f \rho^{2}\right]=\exp \left[\frac{1}{2} i f\right] \sum_{k=0}^{\infty}(2 k+1) i^{k} j_{k}\left(\frac{1}{2} f\right) R_{2 k}^{0}(\rho) .
$$

For the case that the focal factor is given by 
$\exp \left[\frac{i f}{u_{0}}\left(1-\sqrt{1-s_{0}^{2} \rho^{2}}\right)\right]$, there is the result

$$
\begin{aligned}
& \frac{\exp \left[\frac{i f}{u_{0}}\left(1-\sqrt{1-s_{0}^{2} \rho^{2}}\right)\right]}{\sqrt{1-s_{0}^{2} \rho^{2}}}=\frac{\exp \left[i f / u_{0}\right]}{i u_{0}} . \\
& \sum_{k=0}^{\infty}(2 k+1) f j_{k}(f / 2) h_{k}^{(2)}\left(f / 2 v_{0}\right) R_{2 k}^{0}(\rho),
\end{aligned}
$$

where $j_{k}$ and $h_{k}^{(2)}$ are spherical Bessel and Hankel functions, see [23], 10.4.7, of order $k$ and

$$
v_{0}=\frac{1-\sqrt{1-s_{0}^{2}}}{1+\sqrt{1-s_{0}^{2}}},
$$

see Appendix A.

The second step is to determine the $R^{0}$-expansion of the algebraic factor in b. In Appendix B it is shown that for real $\alpha, \beta, \gamma$ and $a, b, c$ the Taylor coefficients $q_{n}$ in

$$
\left(1-a \rho^{2}\right)^{\alpha}\left(1-b \rho^{2}\right)^{\beta}\left(1-c \rho^{2}\right)^{\gamma}=\sum_{n=0}^{\infty} q_{n} \rho^{2 n}
$$

can be determined recursively according to

$$
\begin{gathered}
q_{-2}=q_{-1}=0, \quad q_{0}=1, \\
q_{n+1}=\frac{1}{n+1}\left[\{(n-\alpha) a+(n-\beta) b+(n-\gamma) c\} q_{n}\right. \\
-\{(n-1-\alpha-\beta) a b+(n-1-\alpha-\gamma) a c+ \\
\quad(n-1-\beta-\gamma) b c\} q_{n-1} \\
\left.+(n-2-\alpha-\beta-\gamma) a b c q_{n-2}\right], \quad n=0,1, \ldots .
\end{gathered}
$$

Then in the case that $|a|,|b|,|c|<1$, the power series in Eq. (37) can be converted into a $R^{0}$-series according to, see Appendix $B$,

$$
\sum_{n=0}^{\infty} q_{n} \rho^{2 n}=\sum_{l=0}^{\infty}\left(\sum_{n=l}^{\infty} b_{n}(l) q_{n}\right) R_{2 l}^{0}(\rho),
$$

where

$$
\begin{gathered}
b_{n}(l)=\frac{2 l+1}{l+1} \frac{\left(\begin{array}{c}
n \\
l
\end{array}\right)}{\left(\begin{array}{c}
n+l+1 \\
n
\end{array}\right)}, \\
l=0,1, \ldots, n=l, l+1, \ldots .
\end{gathered}
$$

The $b_{n}(l)$ can be computed recursively as follows. Firstly,

$$
a_{l}:=b_{l}(l)=\frac{1}{\left(\begin{array}{c}
2 l \\
l
\end{array}\right)}, \quad l=0,1, \ldots,
$$

can be computed recursively according to

$$
a_{0}=1 ; \quad a_{l+1}=\frac{l+1}{4 l+2} a_{l}, l=0,1, \ldots,
$$

and then, for $l=0,1, \ldots$, the $b_{n}(l)$ can be computed recursively according to

$$
\begin{aligned}
& b_{n}(l)=a_{l} \\
& b_{n+1}(l)=\frac{(n+1)^{2}}{(n-l+1)(n+l+2)} b_{n}(l), n=l, l+1, \ldots .
\end{aligned}
$$

It turns out that in all cases, except the one with $|j|=2$ and the --sign in the VMML-case, the algebraic function can be manipulated so as to comprise a linear combination of at most two functions of the form Eq. (37). Hence, the $R^{0}$ expansion of this algebraic function can be determined using Eqs. (37)-(40). The situation for the VMML-case, $|j|=2$ and --sign, is not much different, except that manipulation of the algebraic function now leads to $\rho^{-2}$ times a linear combination of at most two functions of the form Eq. (37). This extra factor $\rho^{-2}$ cancels the $\rho^{|j|},|j|=2$, in the integral expression Eq. (32) for $I_{V M M L}$. It should be noted that this second step in the procedure, in this general form, is relatively expensive in terms of computing time, and that one may look for short-cuts per case.

The third step is to determine the $R^{0}$-expansion of the product of the focal factor and the algebraic function from their $R^{0}$-expansions obtained in the first two steps. Thus, given $u_{l}$, $l=0,1, \ldots$, and $v_{k}, k=0,1, \ldots$, one should determine the coefficients $w_{t}$ in

$$
\sum_{l=0}^{\infty} u_{l} R_{2 l}^{0} \sum_{k=0}^{\infty} v_{k} R_{2 k}^{0}=\sum_{t=0}^{\infty} w_{t} R_{2 t}^{0} .
$$

This can be done by using linearization coefficients to write products $R_{2 l}^{0} R_{2 k}^{0}$ as a linear combination of $R_{2 t}^{0}$. Thus one has

$$
R_{2 l}^{0} R_{2 k}^{0}=\sum_{t} A_{2 l, 2 k, 2 t}^{000} R_{2 t}^{0}
$$

so that the $w_{t}$ required in Eq. (45) are given as

$$
w_{t}=\sum_{l, k=0}^{\infty} A_{2 l, 2 k, 2 t}^{000} u_{l} v_{k} .
$$

In Appendix C, the numbers $A$ in Eq. (46) are related to Clebsch-Gordan coefficients, and there is given a very efficient recursive method to compute them, also see Section 5 .

The fourth step is to write the product $\sum_{t} w_{t} R_{2 t}^{0}$ of the two factors in a and $\mathrm{b}$ and of the radial polynomial $R_{n}^{|m|}$ that occurs in $c$ as a linear combination of radial polynomials with the same azimuthal order $m$. For this we use again the to ClebschGordan coefficients related $A$ 's to write

$$
R_{2 t}^{0} R_{n}^{|m|}=\sum_{h} A_{2 t, n, h}^{0 m m} R_{h}^{|m|} .
$$

The fifth and final step is then to write, using again $A$ coefficients, any polynomial $\rho^{|j|} R_{h}^{|m|}$ as in c as a linear combination of radial polynomials of azimuthal order $m+j$. Letting

$$
p=\frac{1}{2}(h-|m|), \quad q=\frac{1}{2}(h+|m|),
$$

this can be done quite generally as follows, see Appendix D. When $m$ and $j$ have the same sign, we have 


$$
\begin{gathered}
\rho^{|j|} R_{h}^{|m|}(\rho)=R_{|j|}^{|j|}(\rho) R_{h}^{|m|}(\rho)=\sum_{s} A_{|j|, h, s}^{j, m, m+j} R_{s}^{|m+j|}(\rho)= \\
\sum_{i=0}^{|j|} \frac{h+|j|-2 i+1}{h+|j|-i+1} \frac{\left(\begin{array}{c}
p \\
i
\end{array}\right)\left(\begin{array}{c}
q+|j|-i \\
q
\end{array}\right)}{\left(\begin{array}{c}
h+|j|-i \\
|j|
\end{array}\right)} R_{h+|j|-2 i}^{|m+j|}(\rho),
\end{gathered}
$$

and when $m$ and $j$ have opposite sign, one should interchange $p$ and $q$ at the right-hand side of Eq. (50). For the cases $|j|=0,1,2$, we get explicitly

$$
\begin{aligned}
|j|=0: & R_{h}^{|m|} \\
|j|=1: & \frac{q+1}{h+1} R_{h+1}^{|m+j|}+\frac{p}{h+1} R_{h-1}^{|m+j|}, \\
|j|=2: & \frac{(q+2)(q+1)}{(h+2)(h+1)} R_{h+2}^{|m+j|}+\frac{2 p(q+1)}{h(h+2)} R_{h}^{|m+j|} \\
& +\frac{p(p+1)}{h(h+1)} R_{h-2}^{|m+j|}
\end{aligned}
$$

for the right-hand sides of Eq. (50) when $m$ and $j$ have the same sign, and one should interchange $p$ and $q$ in Eqs. (51)-(53) when $m$ and $j$ have opposite sign.

With these five steps, we have reached in all cases, except in the case of $|j|=2$ and back propagating waves in the VMMLintegral, a situation that we can use the basic result Eq. (14) of the NZ-theory that we recall here for completeness,

$$
\int_{0}^{1} R_{n}^{|m|}(\rho) J_{m}(2 \pi r \rho) \rho d \rho=(-1)^{\frac{n-m}{2}} \frac{J_{n+1}(2 \pi r)}{2 \pi r},
$$

with azimuthal order of the radial polynomial matching the order of the Bessel function. In the VMML-case, $|j|=2$ and back propagating waves, the $\rho^{|j|},|j|=2$, is cancelled, and this leads to an integral of the type Eq. (54) in which azimuthal order and Bessel function order differ by \pm 2 . These integrals will be considered separately in Sec. 9 and Appendix H.

To summarize, the general I-integral has the form

$$
I=\int_{0}^{1} a(\rho) f(\rho) p(\rho) b(\rho) \rho d \rho,
$$

where
a. $f(\rho)$ is a focal factor,
b. $a(\rho)$ is an algebraic factor,
c. $p(\rho)$ is a polynomial factor $\rho^{|j|} R_{n}^{|m|}(\rho)$,
d. $b(\rho)$ is a Bessel function $J_{m+j}(2 \pi r \rho)$.

The product $a(\rho) f(\rho)$ is expanded as

$$
a(\rho) f(\rho)=\sum_{t} w_{t} R_{2 t}^{0}(\rho),
$$

either directly or by using $A$-coefficients as in Eq. (46) to obtain the $w_{t}$ from the $R^{0}$-expansion of $a$ and $f$. Next the product $a(\rho) f(\rho) R_{n}^{|m|}(\rho)$ is expanded as

$$
a(\rho) f(\rho) R_{n}^{|m|}(\rho)=\sum_{h, t} A_{2 t, n, h}^{0 m m} w_{t} R_{h}^{|m|}(\rho)
$$

using $A$-coefficients to linearize products $R_{2 t}^{0} R_{n}^{|m|}$. Finally, the product $a(\rho) f(\rho) \rho^{|m|} R_{n}^{|m|}(\rho)$ is expanded as

$$
a(\rho) f(\rho) \rho^{|j|} R_{n}^{|m|}(\rho)=\sum_{s, h, t} A_{2 t, n, h}^{0 m m} A_{|j|, h, s}^{j, m, m+j} w_{t} R_{s}^{|m+j|}(\rho)
$$

using $A$-coefficients to linearize products $\rho^{|j|} R_{h}^{|m|}(\rho)$ as a sum of radial polynomials whose azimuthal orders match the order of the Bessel function $J_{m+j}$. Then by the basic result Eq. (54) of the NZ-theory, this yields for I the result

$$
I=\sum_{s, h, t} A_{2 t, n, h}^{0 m m} A_{|j|, h, s}^{j, m, m+j} w_{t}(-1)^{\frac{s-m-j}{2}} \frac{J_{s+1}(2 \pi r)}{2 \pi r} .
$$

In the next sections, the main effort is directed at finding the $R^{0}$-coefficients $w_{t}$ in Eq. (56) for the particular optical settings of Section 3.

\section{DETAILS FOR BASIC LOW-NA, SMALL DEFOCUS SYSTEMS(B)}

We have in this case

$$
I_{\mathrm{B}}=\int_{0}^{1} \exp \left[i f \rho^{2}\right] R_{n}^{|m|}(\rho) J_{m}(2 \pi r \rho) \rho d \rho,
$$

with integer $n$ and $m$ such that $n-|m|$ is even and nonnegative. Using Bauer's formula Eq. (34), the linearization formula Eq. (48), together with the basic result Eq. (54) of the NZtheory, we obtain

$$
I_{\mathrm{B}}=\sum_{h, k}(-1)^{\frac{m-h}{2}} A_{2 k, n, h}^{0 m m} C_{2 k}^{0}(f) B_{h}(r)
$$

where the summation is over all $k=0,1, \ldots$ and all integer $h$ of same parity as $m$ with $h \geq|m|$, and

$$
\begin{aligned}
C_{2 k}^{0}(f) & =(2 k+1) i^{k} \exp \left[\frac{1}{2} i f\right] j_{k}\left(\frac{1}{2} f\right), \\
B_{h}(r) & =\frac{J_{h+1}(2 \pi r)}{2 \pi r} .
\end{aligned}
$$

This result is what one can term the Bessel-Bessel series alternative for the power-Bessel series in Eqs. (16)-(17).

There are the following features, see Appendix $C$ for proofs.

a. We have $A_{2 k, n, h}^{0 m m} \neq 0$ only when

$$
\frac{1}{2}|n-h| \leq k \leq \frac{1}{2}(n+h),
$$

in which $h$ and $k$ are non-negative integers with $h \geq|m|$ and $h$ and $n$ have the same parity (as $m$ ).

b. We have

$$
\begin{aligned}
A_{2 k, n, h}^{0 m m}= & A_{2 k, n, h}^{0,-m,-m}= \\
& (h+1)\left|\left(\begin{array}{ccc}
k & \frac{1}{2} n & \frac{1}{2} h \\
0 & \frac{1}{2} m & -\frac{1}{2} m
\end{array}\right)\right|^{2} \geq 0,
\end{aligned}
$$

where on the right-hand a 3j-symbol, see [23], Chap. 34, appears, and

$$
\sum_{h} A_{2 k, n, h}^{0 m m}=1=\sum_{k} \frac{2 k+1}{h+1} A_{2 k, n, h}^{0 m m} .
$$


c. The $A_{2 k, n, h}^{0 m m}, k=\frac{1}{2}|n-h|, \ldots, \frac{1}{2}(n+h)$ can be computed recursively for given $m, n$ and $h$ as follows. When $0 \leq m \leq h \leq n$, we have

$$
\begin{gathered}
\left(\begin{array}{ccc}
\frac{1}{2}|n-h|-1 & \frac{1}{2} n & \frac{1}{2} h \\
0 & \frac{1}{2} m & -\frac{1}{2} m
\end{array}\right)=0, \\
\left(\begin{array}{ccc}
\frac{1}{2}|n-h| & \frac{1}{2} n & \frac{1}{2} h \\
0 & \frac{1}{2} m & -\frac{1}{2} m
\end{array}\right)=(-1)^{\frac{n-m}{2}}\left(\frac{(n-h) ! h !}{(n+1) !}\right)^{1 / 2} . \\
\left(\frac{\left(\frac{n+m}{2}\right) !\left(\frac{n-m}{2}\right) !}{\left(\frac{h+m}{2}\right) !\left(\frac{h-m}{2}\right) !}\right)^{1 / 2} \frac{1}{\left(\frac{n-h}{2}\right) !},
\end{gathered}
$$

and

$$
\begin{aligned}
& m(2 k+1)\left(\begin{array}{ccc}
k & \frac{1}{2} n & \frac{1}{2} h \\
0 & \frac{1}{2} m & -\frac{1}{2} m
\end{array}\right)=\left(k^{2}-\left(\frac{n-h}{2}\right)^{2}\right)^{1 / 2} \\
& \left(\left(\frac{n+h}{2}+1\right)^{2}-k^{2}\right)^{1 / 2}\left(\begin{array}{ccc}
k-1 & \frac{1}{2} n & \frac{1}{2} h \\
0 & \frac{1}{2} m & -\frac{1}{2} m
\end{array}\right)+ \\
& \left((k+1)^{2}-\left(\frac{n-h}{2}\right)^{2}\right)^{1 / 2}\left(\left(\frac{n+h}{2}+1\right)^{2}-(k+1)^{2}\right)^{1 / 2} \\
& \left(\begin{array}{ccc}
k+1 & \frac{1}{2} n & \frac{1}{2} h \\
0 & \frac{1}{2} m & -\frac{1}{2} m
\end{array}\right)
\end{aligned}
$$

for $k=\frac{1}{2}|n-h|, \frac{1}{2}|n-h|+1, \ldots, \frac{1}{2}(n+h)-1$. When $m \geq 0$ and $h \geq n$, the recursion Eqs. (66)- (68) applies as well, except that in Eq. (67) on the right-hand side $h$ and $n$ should be interchanged. In Figure 3 we indicate the set of $(h, k)$ such that $A$ is non-vanishing (for a fixed value of $m$ and $n$ ) and how the recursion Eqs. (66)-(68) fits into this picture. The evaluation of the double series in Eq. (61) is done most conveniently in accordance with Eq. (64) and the recursion Eqs. (66)-(68).

d. The $j_{k}$ and $J_{h+1}$ required in Eqs. (61)-(62) can be computed recursively according to well-established practices to compute (spherical) Bessel functions.

e. The $C_{2 k}^{0}(f)$ and $B_{h}(r)$ in Eq. (62) are orthogonal, see Appendix $\mathrm{E}$, in the sense that for $k_{1}, k_{2}=0,1, \ldots$

$$
\int_{-\infty}^{\infty} C_{2 k_{1}}^{0}(f) C_{2 k_{2}}^{0 *}(f) d f=2 \pi\left(2 k_{1,2}+1\right) \delta_{k_{1} k_{2}}
$$

and for integer $h_{1}, h_{2}$ at least equal to $|m|$ and of same parity as $|m|$

$$
\int_{0}^{\infty} B_{h_{1}}(r) B_{h_{2}}^{*}(r) r d r=\frac{1}{8 \pi^{2}\left(h_{1,2}+1\right)} \delta_{h_{1} h_{2}} .
$$

f. When $n=m=0$ the double series in Eq. (61) reduces to a series over $h=2 k=0,2, \ldots$, and then we get

$$
I_{\mathrm{B}}=\exp \left[\frac{1}{2} i f\right] \sum_{k=0}^{\infty}(2 k+1)(-i)^{k} j_{k}\left(\frac{1}{2} f\right) \frac{J_{2 k+1}(2 \pi r)}{2 \pi r}
$$

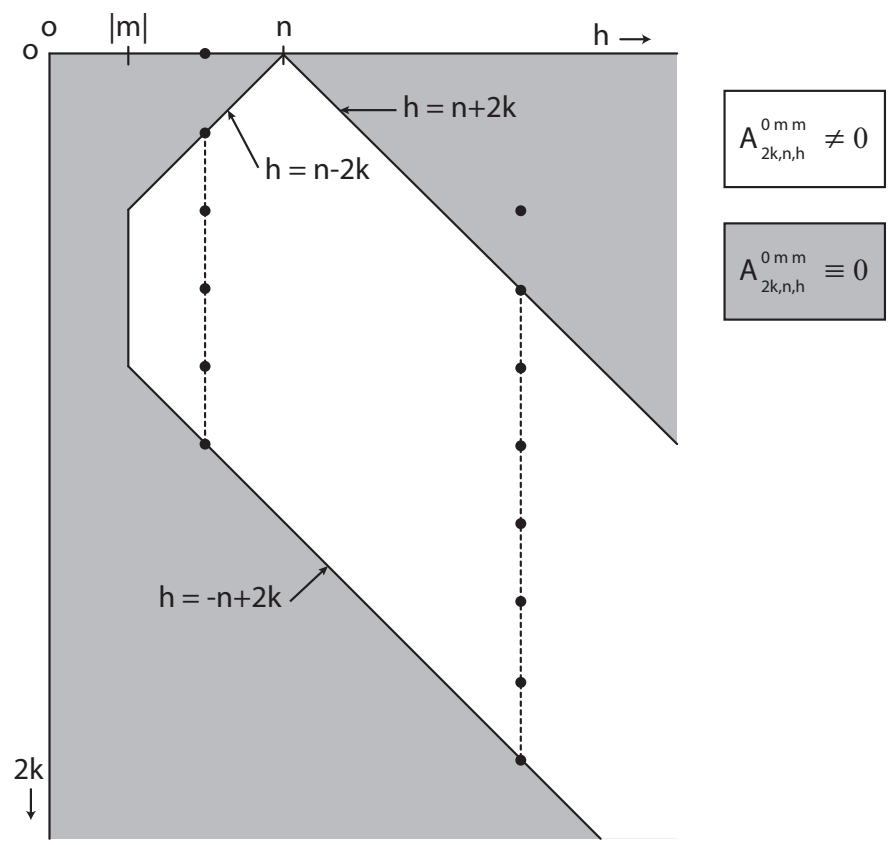

FIG. 3 Given integers $n$ and $m$ with $n-|m|$ even and non-negative, the unshaded set $h \geq|m|,|h-n| \leq 2 k$ contains all points $(h, k)$ with non-negative integer $h$ and $k$ such that $A_{2 k, n, h}^{0 m m} \neq 0$. The $A_{2 k, n, h}^{0 m m}$ with $(h, k)$ in this set can be computed recursively in $k=\frac{1}{2}|n-h|, \frac{1}{2}|n-h|+1, \cdots, \frac{1}{2}|n+h|$ according to Eq. (68) with initialization at $k=\frac{1}{2}|n-h|-1, \frac{1}{2}|n-h|$ given by Eq. (66-67). In all this, $h$ has the same parity as $n$.

which is the Bessel-Bessel series form of Lommel's result in Eq. (18) for the aberration-free case, also see [26]. We note that $I_{\mathrm{B}}$ in Eq. (60) has been evaluated in [25] in BesselBessel series form as well, using an ad hoc procedure for linearizing products $R_{2 k}^{0} R_{n}^{m}$.

g. There are the bounds, valid for real $z$ and $h, k=0,1, \ldots$,

$$
\left|\frac{J_{h+1}(z)}{z}\right| \leq \frac{1}{h+1}, \quad\left|j_{k}(z)\right| \leq \frac{1}{\sqrt{2 k+1}},
$$

see [23], 10.6.1 and 10.4.1, and [23], 10.6.12. Furthermore, there is for fixed $z$ the asymptotics

$$
J_{v}(z) \sim \frac{1}{\sqrt{2 \pi v}}\left(\frac{e z}{2 v}\right)^{v}, \quad v \rightarrow \infty
$$

When we use Eq. (73) for $J_{h+1}(2 \pi r)$ and $j_{k}\left(\frac{1}{2} f\right)=$ $2(\pi f)^{-1 / 2} J_{k+1 / 2}\left(\frac{1}{2} f\right)$, together with the bounds on $A$ that follow from Eq. (64) and Eq. (65), it is seen that there is hardly any loss-of-digits in the series in Eq. (61). Furthermore, the summations in Eq. (61) can be limited to values of $h$ up to a little beyond $\pi e r$ and values of $k$ up to a little beyond $\frac{1}{4} e f$.

\section{DETAILS FOR SYSTEMS WITH LOW NA AND LARGE DEFOCUS (BLD)}

We consider in this case

$$
\begin{aligned}
& I_{\mathrm{BLD}}= \\
& \int_{0}^{1} \exp \left[\frac{i f}{u_{0}}\left(1-\sqrt{1-s_{0}^{2} \rho^{2}}\right)\right] R_{n}^{|m|}(\rho) J_{m}(2 \pi r \rho) \rho d \rho
\end{aligned}
$$


and

$$
\begin{aligned}
& \int_{0}^{\bar{I}_{\mathrm{BLD}}}= \\
& \int_{0}^{1} \frac{\exp \left[\frac{i f}{u_{0}}\left(1-\sqrt{1-s_{0}^{2} \rho^{2}}\right)\right]}{\sqrt{1-s_{0}^{2} \rho^{2}}} R_{n}^{|m|}(\rho) J_{m}(2 \pi r \rho) \rho d \rho
\end{aligned}
$$

For $\bar{I}_{\mathrm{BLD}}$ in $\left(74_{\mathrm{b}}\right)$, we can directly use the $R^{0}$-expansion in Eq. (35), and then we are at once in the same position as in Sec. 5, except that in Eq. (62) we should replace $C_{2 k}^{0}(f)$ by

$$
D_{2 k}^{0}(f)=\frac{\exp \left[i f / u_{0}\right]}{i u_{0}}(2 k+1) f j_{k}(f / 2) h_{k}^{(2)}\left(f / 2 v_{0}\right)
$$

and use that in the series in Eq. (61). The only issue is now how this changes the convergence behaviour of Eq. (61).

The quantity in Eq. (75) is the $R_{2 k}^{0}$-coefficient of the function at the left-hand side of Eq. (35). By the Cauchy-Schwarz inequality and the identities

$$
\begin{aligned}
& \int_{0}^{1}\left|R_{2 k}^{0}(\rho)\right|^{2} \rho d \rho=\frac{1}{2(2 k+1)}, \\
& \int_{0}^{1} \frac{\rho d \rho}{1-s_{0}^{2} \rho^{2}}=\frac{-1}{2 s_{0}^{2}} \ln \left(1-s_{0}^{2}\right),
\end{aligned}
$$

it is seen that the modulus of Eq. (75) does not exceed

$$
\frac{1}{s_{0}}\left(-(2 k+1) \ln \left(1-s_{0}^{2}\right)\right)^{1 / 2} \text {. }
$$

Furthermore, there is for fixed $z$ the asymptotics

$$
\begin{gathered}
j_{k}(z) \sim \frac{z^{k}}{1 \cdot 3 \cdot \ldots \cdot(2 k+1)}, \\
y_{k}(z) \sim-\frac{1 \cdot 3 \cdot \ldots \cdot(2 k-1)}{z^{k+1}}
\end{gathered}
$$

as $k \rightarrow \infty$, see [23], 10.53. Using this with

$$
j_{k}(f / 2) \text { and } h_{k}^{(2)}\left(f / 2 v_{0}\right)=j_{k}\left(f / 2 v_{0}\right)-i y_{k}\left(f / 2 v_{0}\right) \text {, }
$$

it is seen that the quantity in Eq. (75) has large- $k$ behaviour

$$
\frac{2}{u_{0}} \exp \left[i f / u_{0}\right] v_{0}^{k+1}
$$

With $v_{0}$ given in Eq. (36), it is a fact that $v_{0}$ is well below 1, unless $s_{0}$ gets very close to 1 . For instance, $s_{0}=0.8$ gives $v_{0}=1 / 4, s_{0}=0.95$ gives $v_{0}=0.52$ and $s_{0}=0.99$ gives $v_{0}=0.75$.

For $I_{\mathrm{BLD}}$ in Eq. (74a), differentiate Eq. (35) with respect to $f$ to obtain the $R^{0}$-expansion

$$
\begin{aligned}
& \exp \left[\frac{i f}{u_{0}}\left(1-\sqrt{1-s_{0}^{2} \rho^{2}}\right)\right]=\sum_{k=0}^{\infty} E_{2 k}^{0}(f) R_{2 k}^{0}(\rho)= \\
& \exp \left[\frac{i f}{u_{0}}\right] \sum_{k=0}^{\infty}(2 k+1) \frac{d}{d f}\left[f j_{k}(f / 2) h_{k}^{(2)}\left(f / 2 v_{0}\right)\right] R_{2 k}^{0}(\rho)
\end{aligned}
$$

of the focal factor in Eq. (22) and Eq. (74a). Using the recursive properties of the spherical Bessel and Hankel functions and their derivatives, see [23], 10.51, it will be shown in Appendix $\mathrm{F}$ that (with $h=h^{(1)}$ or $h^{(2)}$ at both sides)

$$
\frac{d}{d f}\left[f j_{k}(f / 2) h_{k}\left(f / 2 v_{0}\right)\right]=\frac{f}{2} j_{k-1} h_{k}-\frac{f}{2 v_{0}} j_{k} h_{k+1},
$$

where on the right-hand side the $j$ 's have argument $f / 2$ and the $h$ 's have argument $f / 2 v_{0}$. We are now again in the same position as in Section 5 when we take $E_{2 k}^{0}(f)$ of Eq. (81) rather than $C_{2 k}^{0}(f)$ of Eq. (62) in the series in Eq. (61). From Eq. (82) it is also seen that the convergence behaviour of the resulting double series for $\bar{I}_{\mathrm{BLD}}$ is roughly the same as that of the double series for $I_{\mathrm{BLD}}$.

\section{DETAILS FOR SYSTEMS WITH HIGH NA AND VECTOR FIELDS (V)}

We consider in this case

$$
\begin{gathered}
I_{\mathrm{V}}=\int_{0}^{1}\left(1+\sqrt{1-s_{0}^{2} \rho^{2}}\right)^{-|j|+1} \exp \left[\frac{i f}{u_{0}}\left(1-\sqrt{1-s_{0}^{2} \rho^{2}}\right)\right] . \\
\rho^{|j|} R_{n}^{|m|}(\rho) J_{m+j}(2 \pi r \rho) \rho d \rho
\end{gathered}
$$

and

$$
\begin{gathered}
\bar{I}_{\mathrm{V}}=\int_{0}^{1} \frac{\left(1+\sqrt{1-s_{0}^{2} \rho^{2}}\right)^{-|j|+1}}{\left(1-s_{0}^{2} \rho^{2}\right)^{1 / 4}} \exp \left[\frac{i f}{u_{0}}\left(1-\sqrt{1-s_{0}^{2} \rho^{2}}\right)\right] . \\
\rho^{|j|} R_{n}^{|m|}(\rho) J_{m+j}(2 \pi r \rho) \rho d \rho
\end{gathered}
$$

where $j=-2,-1,0,1,2$. The distinction between Eq. (83) and Eq. (84) is reflected by the factor $\left(1-s_{0}^{2} \rho^{2}\right)^{-1 / 4}$ and has to do with how one wants to handle the radiometric effect, as discussed in Subsection 3.3.

The treatment of the $I_{V}$-integral follows the approach in Section 6 for the $I_{B L D}$-integral closely. The treatment of the $\bar{I}_{\mathrm{V}^{-}}$ integrals is more complicated since Zernike expansion of the algebraic factor in front of the focal factor must be done via a power series expansion, see Eqs. (37)-(44), and this expansion has to be combined with the Zernike expansion of the focal factor. We therefore proceed by first considering the $I_{\mathrm{V}^{-}}$ integral.

In the case that $|j|=1$, we have

$$
\begin{aligned}
& I_{\mathrm{V}}= \\
& \int_{0}^{1} \exp \left[\frac{i f}{u_{0}}\left(1-\sqrt{1-s_{0}^{2} \rho^{2}}\right)\right] \rho R_{n}^{|m|}(\rho) J_{m+j}(2 \pi r \rho) \rho d \rho .
\end{aligned}
$$

Inserting the $R^{0}$-expansion Eq. (81) of the focal factor into Eq. (84) and linearizing products $R_{2 k}^{0} R_{n}^{|m|}$ using $A$-coefficients, we get

$$
I_{\mathrm{V}}=\sum_{h, k} A_{2 k, n, h}^{0 m m} E_{2 k}^{0}(f) \int_{0}^{1} \rho R_{h}^{|m|}(\rho) J_{m+j}(2 \pi r \rho) \rho d \rho .
$$

In the case that $|j|=0$, we proceed as in the case $|j|=1$, except that we now need the $R^{0}$-expansion

$$
\begin{gathered}
\left(1+\sqrt{1-s_{0}^{2} \rho^{2}}\right) \exp \left[\frac{i f}{u_{0}}\left(1-\sqrt{1-s_{0}^{2} \rho^{2}}\right)\right]= \\
\sum_{k=0}^{\infty} F_{2 k}^{0}(f) R_{2 k}^{0}(\rho) .
\end{gathered}
$$


This $R^{0}$-expansion can be obtained from Eq. (35) in the same way as the $R^{0}$-expansion in Eq. (81), with the result

$$
\begin{aligned}
& F_{2 k}^{0}(f)=E_{2 k}^{0}(f)+ \\
& \quad i u_{0} \exp \left[\frac{i f}{u_{0}}\right](2 k+1)\left(\frac{d}{d f}\right)^{2}\left[f j_{k}(f / 2) h_{k}^{(2)}\left(f / 2 v_{0}\right)\right] .
\end{aligned}
$$

Using the recursive properties of the spherical Bessel and Hankel functions and their derivatives, it will be shown in Appendix F that (with $h=h^{(1)}$ or $h^{(2)}$ )

$$
\begin{aligned}
& \left(\frac{d}{d f}\right)^{2}\left[f j_{k}(f / 2) h_{k}\left(f / 2 v_{0}\right)\right]=\frac{k+1}{v_{0}} j_{k} h_{k-1}+ \\
& k j_{k-1} h_{k}-\frac{f}{4 v_{0}^{2}} j_{k} h_{k}-\frac{f}{4} j_{k} h_{k}-\frac{f}{2 v_{0}} j_{k-1} h_{k+1},
\end{aligned}
$$

where on the second line of Eq. (88) all $j$ 's have argument $f / 2$ while all $h$ 's have argument $f / 2 v_{0}$. Thus we get a similar result for the case of $I_{\mathrm{V}}$ with $|j|=0$ as in Eq. (85), with $F_{2 k}^{0}(f)$ instead of $E_{2 k}^{0}(f)$, and with remaining integrals

$$
\int_{0}^{1} R_{h}^{|m|}(\rho) J_{m}(2 \pi r \rho) \rho d \rho=(-1)^{\frac{h-m}{2}} B_{h}(r) .
$$

Finally, in the case $|j|=2$ in Eq. (83), we proceed as in the two previous cases, where we now need the $R^{0}$-expansion

$$
\begin{gathered}
\left(1+\sqrt{1-s_{0}^{2} \rho^{2}}\right)^{-1} \exp \left[\frac{i f}{u_{0}}\left(1-\sqrt{1-s_{0}^{2} \rho^{2}}\right)\right]= \\
\sum_{k=0}^{\infty} G_{2 k}^{0}(f) R_{2 k}^{0}(\rho) .
\end{gathered}
$$

We shall show in Appendix $G$ that

$$
\begin{aligned}
& G_{2 k}^{0}(f)= \frac{1}{i u_{0}} \exp \left[\frac{i f}{u_{0}}\right] . \\
&\left(\frac{d_{k}}{2 k+2}+\sum_{l=k+1}^{\infty} \frac{(-1)^{k-l}(2 k+1) d_{l}}{2 l(l+1)}\right), \\
& k=0,1, \ldots,
\end{aligned}
$$

where

$$
d_{k}=(2 k+1)\left\{1+\frac{i f}{u_{0}}+f \frac{d}{d f}\right\}\left[f j_{k}(f / 2) h_{k}^{(2)}\left(f / 2 v_{0}\right)\right] \text {. }
$$

Then we proceed as before and we get for $I_{\mathrm{V}}$ with $|j|=2$ the result Eq. (85), with $G_{2 k}^{0}(f)$ instead of $E_{2 k}^{0}(f)$, and with remaining integrals

$$
\int_{0}^{1} \rho^{2} R_{h}^{|m|}(\rho) J_{m+j}(2 \pi r \rho) \rho d \rho .
$$

The remaining integrals in Eq. (85) and Eq. (93) can be expressed in terms of Jinc functions using Eqs. (49)-(53) and the basic integral result in Eq. (54).

We next consider $\bar{I}_{\mathrm{V}}$ in Eq. (84). The factor $\left(1-s_{0}^{2} \rho^{2}\right)^{-1 / 4}$ prevents a direct $R^{0}$-expansion of the product of the algebraic function and the focal factor. We now write

$$
\begin{gathered}
\frac{\left(1+\sqrt{1-s_{0}^{2} \rho^{2}}\right)^{-|j|+1}}{\left(1-s_{0}^{2} \rho^{2}\right)^{1 / 4}} \exp \left[\frac{i f}{u_{0}}\left(1-\sqrt{1-s_{0}^{2} \rho^{2}}\right)\right]= \\
\left(1-s_{0}^{2} \rho^{2}\right)^{1 / 4}\left(1+\sqrt{1-s_{0}^{2} \rho^{2}}\right)^{-|j|+1} . \\
\frac{\exp \left[\frac{i f}{u_{0}}\left(1-\sqrt{1-s_{0}^{2} \rho^{2}}\right)\right]}{\sqrt{1-s_{0}^{2} \rho^{2}}} .
\end{gathered}
$$

For the fraction involving the focal factor on the last line of Eq. (94) we use the $R^{0}$-expansion $\sum_{k} v_{k} R_{2 k}^{0}$ in Eq. (35). As to the algebraic function

$$
\left(1-s_{0}^{2} \rho^{2}\right)^{1 / 4}\left(1+\sqrt{1-s_{0}^{2} \rho^{2}}\right)^{-|j|+1}
$$

on the last line of Eq. (94), we proceed case-by-case. Thus

$$
\begin{aligned}
& |j|=0:\left(1-s_{0}^{2} \rho^{2}\right)^{1 / 4}+\left(1-s_{0}^{2} \rho^{2}\right)^{3 / 4} ; \\
& |j|=1:\left(1-s_{0}^{2} \rho^{2}\right)^{1 / 4}
\end{aligned}
$$

and for $|j|=2$, we rewrite

$$
\begin{aligned}
& \left(1-s_{0}^{2} \rho^{2}\right)^{1 / 4}\left(1+\sqrt{1-s_{0}^{2} \rho^{2}}\right)^{-1}= \\
& \left(1-s_{0}^{2} \rho^{2}\right)^{1 / 4} \frac{1}{s_{0}^{2} \rho^{2}}\left(1-\sqrt{1-s_{0}^{2} \rho^{2}}\right)= \\
& \frac{1}{s_{0}^{2}} \frac{1}{\rho^{2}}\left[\left(1-s_{0}^{2} \rho^{2}\right)^{1 / 4}-\left(1-s_{0}^{2} \rho^{2}\right)^{3 / 4}\right] .
\end{aligned}
$$

The power series $\sum_{n=0}^{\infty} r_{n} \rho^{2 n}$ of each of the functions $\left(1-s_{0}^{2} \rho^{2}\right)^{\alpha}$ occurring in Eq. (96) and Eq. (97) is determined using Eqs. (37)-(39) with $a=s_{0}^{2}, b=\beta=c=\gamma=0$. The recursive scheme Eqs. (38)-(39) for computing the $r_{n}$ simplifies to

$$
r_{0}=1 ; \quad r_{n+1}=\frac{n-\alpha}{n+1} r_{n}, n=0,1, \ldots .
$$

Then we get the power series for the cases $|j|=0,1$ in Eq. (95) directly, and the one for $|j|=2$ is obtained by observing that the quantity in [ ] in Eq. (97) vanishes at $\rho=0$. The power series expansion $\sum_{n=0}^{\infty} q_{n} \rho^{2 n}$ of the algebraic function in Eq. (95) is subsequently converted into an $R^{0}$-series $\sum_{l=0}^{\infty} u_{l} R_{2 l}^{0}$ according to Eq. (40) with $b_{n}(l)$ computed recursively according to Eqs. (41)-(44). Finally, the whole expression in Eq. (94) is expanded as an $R^{0}$-series $\sum_{t=0}^{\infty} w_{t} R_{2 t}^{0}$ by proceeding as in Eqs. (45)-(47) using Clebsch-Gordan related coefficients $A$ to linearize products $R_{2 l}^{0} R_{2 k}^{0}$. In this manner, we get $R^{0}$-expansions

$$
\begin{aligned}
& \frac{\left(1+\sqrt{1-s_{0}^{2} \rho^{2}}\right)^{-|j|+1}}{\left(1-s_{0}^{2} \rho^{2}\right)^{1 / 4}} \exp \left(\frac{i f}{u_{0}}\left(1-\sqrt{1-s_{0}^{2} \rho^{2}}\right)\right]= \\
& \sum_{t=0}^{\infty} H_{2 t, j}^{0}(f) R_{2 t}^{0}(\rho), \quad|j|=0,1,2
\end{aligned}
$$

of the product of algebraic function times focal factor in the integrand in Eq. (84) for $\bar{I}_{\mathrm{V}}$. From this point onwards we can proceed as in the case of $I_{\mathrm{V}}$-integral by linearizing products $R_{2 t}^{0} R_{n}^{|m|}$ and by writing $\rho^{|j|} R_{n}^{|m|}(\rho)$ as a linear combination of radial polynomials of azimuthal order $m+j$ so as to match the order $m+j$ of the Bessel function occurring in the integrand in Eq. (84).

\section{DETAILS FOR SYSTEMS WITH HIGH NA, VECTOR FIELDS AND MAGNIFICATION (VM)}

We now consider the integral

$$
I_{\mathrm{VM}}=\int_{0}^{1} \frac{\left\{\left(1-s_{0}^{2} \rho^{2}\right)^{1 / 2}+\left(1-s_{0, M}^{2} \rho^{2}\right)^{1 / 2}\right\}^{-|j|+1}}{\left(1-s_{0}^{2} \rho^{2}\right)^{1 / 4}\left(1-s_{0, M}^{2} \rho^{2}\right)^{3 / 4}} .
$$




$$
\exp \left[\frac{i f}{u_{0}}\left(1-\sqrt{1-s_{0}^{2} \rho^{2}}\right)\right] \rho^{|j|} R_{n}^{|m|}(\rho) J_{m+j}(2 \pi r \rho) \rho d \rho
$$

The algebraic function in the integrand in Eq. (100) is now so complicated that deletion of the factor $\left(1-s_{0}^{2} \rho^{2}\right)^{-1 / 4}$ still does not give rise to a situation where the product of the algebraic function and the focal factor can be expanded directly as an $R^{0}$-series. Hence, we have included this factor $\left(1-s_{0}^{2} \rho^{2}\right)^{-1 / 4}$ in the integrand in Eq. (100) right-away.

We basically proceed as in the $\bar{I}_{\mathrm{V}}$-case, and we write

$$
\begin{gathered}
\frac{\left\{\left(1-s_{0}^{2} \rho^{2}\right)^{1 / 2}+\left(1-s_{0, M}^{2} \rho^{2}\right)^{1 / 2}\right\}^{-|j|+1}}{\left(1-s_{0}^{2} \rho^{2}\right)^{1 / 4}\left(1-s_{0, m}^{2} \rho^{2}\right)^{3 / 4}} . \\
\exp \left[\frac{i f}{u_{0}}\left(1-\sqrt{1-s_{0}^{2} \rho^{2}}\right)\right]=\frac{\left(1-s_{0}^{2} \rho^{2}\right)^{1 / 4}}{\left(1-s_{0, M}^{2} \rho^{2}\right)^{3 / 4}} \\
\left\{\left(1-s_{0}^{2} \rho^{2}\right)^{1 / 2}+\left(1-s_{0, M}^{2} \rho^{2}\right)^{1 / 2}\right\}^{-|j|+1} . \\
\quad \frac{\exp \left[\frac{i f}{u_{0}}\left(1-\sqrt{1-s_{0}^{2} \rho^{2}}\right)\right]}{\sqrt{1-s_{0}^{2} \rho^{2}}} .
\end{gathered}
$$

The algebraic function

$$
\frac{\left(1-s_{0}^{2} \rho^{2}\right)^{1 / 4}}{\left(1-s_{0, M}^{2} \rho^{2}\right)^{3 / 4}}\left\{\left(1-s_{0}^{2} \rho^{2}\right)^{1 / 2}+\left(1-s_{0, M}^{2} \rho^{2}\right)^{1 / 2}\right\}^{-|j|+1}
$$

is expanded as an $R^{0}$-series by a case-by-case consideration. Thus we have as in Eqs. (95)-(97)

$$
\begin{aligned}
|j|=0: & \left(1-s_{0}^{2} \rho^{2}\right)^{3 / 4}\left(1-s_{0, M}^{2} \rho^{2}\right)^{-3 / 4} \\
& +\left(1-s_{0}^{2} \rho^{2}\right)^{1 / 4}\left(1-s_{0, M}^{2} \rho^{2}\right)^{-1 / 4} \\
|j|=1: & \left(1-s_{0}^{2} \rho^{2}\right)^{1 / 4}\left(1-s_{0, M}^{2} \rho^{2}\right)^{-3 / 4} \\
|j|=2: & \frac{1}{\left(s_{0, M}^{2}-s_{0}^{2}\right) \rho^{2}}\left[\left(1-s_{0}^{2} \rho^{2}\right)^{3 / 4}\left(1-s_{0, M}^{2} \rho^{2}\right)^{-3 / 4}\right. \\
& \left.-\left(1-s_{0}^{2} \rho^{2}\right)^{1 / 4}\left(1-s_{0, M}^{2} \rho^{2}\right)^{-1 / 4}\right]
\end{aligned}
$$

The power series $\sum_{n=0}^{\infty} r_{n} \rho^{2 n}$ of each of the function $\left(1-s_{0}^{2} \rho^{2}\right)^{\alpha}\left(1-s_{0, M}^{2} \rho^{2}\right)^{\beta}$ occurring in (99)-(101) is determined using Eqs. (37)-(39) with $a=s_{0}^{2}, b=s_{0, M}^{2}$ and $c=\gamma=0$. The recursive scheme Eqs. (38)-(39) for computing the $r_{n}$ simplifies to

$$
\begin{aligned}
& r_{-1}=0, \quad r_{0}=1 ; \quad r_{n+1}=\frac{1}{n+1} . \\
& {\left[((n-\alpha) a+(n-\beta) b) r_{n}-(n-1-\alpha-\beta) a b r_{n-1}\right]} \\
& n=0,1, \ldots
\end{aligned}
$$

Then we get the power series of Eq. (102) for the cases $|j|=0,1$ directly, and the one for the case $|j|=2$ after the observation that the quantity in [ ] in (101) vanishes at $\rho=0$. From this point onwards, we can proceed in entirely the same way as we did in the $\bar{I}_{\mathrm{V}}$-case.

\section{DETAILS FOR SYSTEMS WITH HIGH NA, VECTOR FIELDS, MAGNIFICATION AND MULTI-LAYERED FOCAL REGION (VMML)}

We consider now the integral

$$
I_{\mathrm{VMML}}=\int_{0}^{1} \frac{\left\{\left(1-s_{0, h}^{2} \rho^{2}\right)^{1 / 2} \pm\left(1-s_{0, M}^{2} \rho^{2}\right)^{1 / 2}\right\}^{-|j|+1}}{\left(1-s_{0}^{2} \rho^{2}\right)^{1 / 4}\left(1-s_{0, M}^{2} \rho^{2}\right)^{3 / 4}} .
$$

$$
\begin{aligned}
& \exp \left[\frac{\text { if }}{u_{0, h}}\left(1-\sqrt{1-s_{0, h}^{2} \rho^{2}}\right)\right] . \\
& \rho^{|j|} R_{n}^{|m|}\left(\rho J_{m+j}(2 \pi r \rho) \rho d \rho .\right.
\end{aligned}
$$

For the case of forward propagating waves where the +sign is taken in the quantity between \{\} in the integrand in Eq. (107), we can proceed in entirely the same way as we did for the $\bar{I}_{\mathrm{V}}$-case in Section 7 and the $I_{\mathrm{VM}}$-case in Section 8 . We thus move a factor $\left(1-s_{0, h}^{2} \rho^{2}\right)^{-1 / 2}$ to the focal factor, so as to take advantage of the $R^{0}$-expansion in Eq. (35), and we consider the remaining algebraic function

$$
\begin{aligned}
& \frac{\left(1-s_{0, h}^{2} \rho^{2}\right)^{1 / 2}}{\left(1-s_{0}^{2} \rho^{2}\right)^{1 / 4}\left(1-s_{0, M}^{2} \rho^{2}\right)^{3 / 4}} \cdot \\
& \quad\left\{\left(1-s_{0, h}^{2} \rho^{2}\right)^{1 / 2}+\left(1-s_{0, M}^{2} \rho^{2}\right)^{1 / 2}\right\}^{-|j|+1}
\end{aligned}
$$

case-by-case. Thus we have

$$
\begin{aligned}
|j|=0: & \frac{1-s_{0, h}^{2} \rho^{2}}{\left(1-s_{0}^{2} \rho^{2}\right)^{1 / 4}\left(1-s_{0, M}^{2} \rho^{2}\right)^{3 / 4}}+ \\
& \frac{\left(1-s_{0, h}^{2} \rho^{2}\right)^{1 / 2}}{\left(1-s_{0}^{2} \rho^{2}\right)^{1 / 4}\left(1-s_{0, M}^{2} \rho^{2}\right)^{1 / 4}}, \\
|j|=1: & \frac{\left(1-s_{0, h}^{2} \rho^{2}\right)^{1 / 2}}{\left(1-s_{0}^{2} \rho^{2}\right)^{1 / 4}\left(1-s_{0, M}^{2} \rho^{2}\right)^{3 / 4}}, \\
|j|=2: & \frac{1}{\left(s_{0, M}^{2}-s_{0, h}^{2}\right) \rho^{2}}\left[\frac{1-s_{0, h}^{2} \rho^{2}}{\left(1-s_{0}^{2} \rho^{2}\right)^{1 / 4}\left(1-s_{0, M}^{2} \rho^{2}\right)^{3 / 4}}\right. \\
& \left.-\frac{\left(1-s_{0, h}^{2} \rho^{2}\right)^{1 / 2}}{\left(1-s_{0}^{2} \rho^{2}\right)^{1 / 4}\left(1-s_{0, M}^{2} \rho^{2}\right)^{1 / 4}}\right]
\end{aligned}
$$

Since $0 \leq s_{0}, s_{0, h}, s_{0, M}<1$, the power series of each of the functions in (105)-(107) can be determined by using for the constituent functions $\left(1-s_{0}^{2} \rho^{2}\right)^{\alpha}\left(1-s_{0, h}^{2} \rho^{2}\right)^{\beta}\left(1-s_{0, M}^{2} \rho^{2}\right)^{\gamma}$ occurring in (105-107) the full three-factors instance of the recursion in Eqs. (37)-(39). These power series are subsequently converted into $R^{0}$-series, and from this point onwards we can proceed as earlier.

The case of backward propagating waves where the --sign, instead of the +-sign, is taken in the quantity between \{\} in the integrand in Eq. (107) can be dealt with in entirely the same way when $|j|=0,1$. The case $|j|=2$, however, presents a complication, for then we get, after moving the factor $\left(1-s_{0, h}^{2} \rho^{2}\right)^{-1 / 2}$ to the focal factor, the expression (111) with a +-sign, instead of a --sign, between the two terms in 
[ ]. Hence, the $\rho^{-2}$ in front of this expression in [ ] is not canceled and cancels, instead, the $\rho^{|j|}(|j|=2)$ in the integrand in Eq. (107). Thus we have now

$I_{\mathrm{VMML}}=\int_{0}^{1} \frac{1}{s_{0, M}^{2}-s_{0, h}^{2}}\left[\frac{1-s_{0, h}^{2} \rho^{2}}{\left(1-s_{0}^{2} \rho^{2}\right)^{1 / 4}\left(1-s_{0, M}^{2} \rho^{2}\right)^{3 / 4}}+\right.$

$\left.\frac{\left(1-s_{0}^{2} \rho^{2}\right)^{1 / 2}}{\left(1-s_{0}^{2} \rho^{2}\right)^{1 / 4}\left(1-s_{0, M}^{2} \rho^{2}\right)^{1 / 4}}\right] \exp \left[\frac{i f}{u_{0, h}}\left(1-\sqrt{1-s_{0, h}^{2} \rho^{2}}\right)\right]$.

$$
R_{n}^{|m|}(\rho) J_{m+j}(2 \pi r \rho) \rho d \rho
$$

with $|j|=2$. Expanding the product of the algebraic function and the focal factor in an $R^{0}$-series $\sum_{t} w_{t} R_{2 t}^{0}$ in the same way as we did for the earlier cases, we get now

$$
I_{\mathrm{VMML}}=\sum_{t=0}^{\infty} w_{t} \int_{0}^{1} R_{2 t}^{0}(\rho) R_{n}^{|m|}(\rho) J_{m \pm 2}(2 \pi r \rho) \rho d \rho,
$$

where the \pm -sign at the right-hand side of Eq. (113) corresponds to $j= \pm 2$ in Eq. (112). To evaluate the remaining integrals, we write

$$
R_{2 t}^{0} R_{h}^{|m|}=\sum_{h} A_{2 t, n, h}^{0 m m} R_{h}^{|m|}
$$

as usual, and we are left with

$$
I_{\mathrm{VMML}}=\sum_{t, h} w_{t} A_{2 t, n, h}^{0 m m} I_{m h}^{ \pm},
$$

where for integer $m$ and $h$ with $h-|m|$ even and non-negative

$$
I_{m h}^{ \pm}=\int_{0}^{1} R_{h}^{|m|}(\rho) J_{m \pm 2}(2 \pi r \rho) \rho d \rho .
$$

Note that azimuthal order $m$ of the radial polynomial and order $m \pm 2$ of the Bessel function do not match, and so we cannot apply the basic integral result Eq. (54) directly. In Appendix $\mathrm{H}$ the following will be shown. We have

$$
\begin{aligned}
I_{m h}^{ \pm} & =(-1)^{|m|} I_{|m|, h}^{\mp}, \quad m<0, \\
I_{0 h}^{ \pm} & =I_{0 h}^{+},
\end{aligned}
$$

and for $m=1,2, \ldots$ with $p=\frac{1}{2}(h-m)$ and $q=\frac{1}{2}(h+m)$

$$
\begin{aligned}
& I_{m h}^{-}=-(-1)^{p} \frac{p+1}{q} \frac{J_{h+1}(2 \pi r)}{2 \pi r}+ \\
& (-1)^{p} \sum_{k=1}^{p+1} \frac{(m-1)(h-2 k+1)}{q(p+1)} \frac{\left(\begin{array}{c}
p+1 \\
k
\end{array}\right)}{\left(\begin{array}{c}
q-1 \\
k
\end{array}\right)} \frac{J_{h-2 k+1}(2 \pi r)}{2 \pi r},
\end{aligned}
$$

while for $m=0,1, \ldots$

$$
\begin{aligned}
I_{m h}^{+}= & -(-1)^{p} \frac{p}{q+1} \frac{J_{h+1}(2 \pi r)}{2 \pi r}+ \\
& (-1)^{p} \sum_{s=0}^{\infty} \frac{(m+1)(h+2 s+3)}{(p+s+1)(q+s+2)} . \\
& \frac{\left(\begin{array}{c}
p+s+1 \\
p
\end{array}\right)}{\left(\begin{array}{c}
q+s+1 \\
q
\end{array}\right)} \frac{J_{h+2 s+3}(2 \pi r)}{2 \pi r} .
\end{aligned}
$$

The coefficients

$$
\begin{aligned}
& b_{h k}=\frac{(m-1)(h-2 k+1)}{q(p+1)} \frac{\left(\begin{array}{c}
p+1 \\
k
\end{array}\right)}{\left(\begin{array}{c}
q-1 \\
k
\end{array}\right)}, \\
& k=1, \ldots, p+1,
\end{aligned}
$$

in Eq. (119) can be computed recursively according to

$$
\begin{aligned}
& b_{h 1}=\frac{(m-1)(h-1)}{q(q-1)} ; \\
& b_{h, k+1}=\frac{h-2 k-1}{h-2 k+1} \frac{p-k+1}{q-k+1} b_{h k}, k=1, \ldots, p,
\end{aligned}
$$

where we observe that all $b_{h k}$ vanish when $m=1$. The coefficients

$$
\begin{aligned}
& c_{h s}=\frac{(m+1)(h+2 s+3)}{(p+s+1)(q+s+2)} \frac{\left(\begin{array}{c}
p+s+1 \\
p
\end{array}\right)}{\left(\begin{array}{c}
q+s+1 \\
q
\end{array}\right)}, \\
& s=0,1, \ldots,
\end{aligned}
$$

can be computed recursively according to

$$
\begin{aligned}
& c_{h 0}=\frac{(m+1)(h+3)}{(q+1)(q+2)} ; \\
& c_{h, s+1}=\frac{h+2 s+5}{h+2 s+3} \frac{p+s+1}{q+s+3} c_{h s}, \\
& s=0,1, \ldots .
\end{aligned}
$$

Since $h=q+p$ and $q \geq p$, it follows easily from Eq. (124) that $0 \leq c_{h, s+1} \leq c_{h s}$. Moreover, to the Bessel series in Eq. (120) the bounds and asymptotics in Eqs. (72)-(73) apply, and it follows that there is hardly any loss-of-digits and that including all terms with $s$ up to a little beyond $\pi e r$ is generally enough for sufficient accuracy. The decay behaviour of $I_{m h}^{-}$in Eq. (119) is a more delicate issue, see Figure 6.

With the $I_{m h}^{ \pm}$being computed, we can return to Eq. (115) and from there we can proceed as usual to compute $I_{\mathrm{VMML}}$.

\section{IMPLEMENTATION, COMPUTATION AND CONVERGENCE}

In this section we comment on the implementation, computation and convergence properties of the new advanced scheme to compute ENZ-diffraction integrals. Where computation times are reported, these pertain to the algorithms presented in this paper implemented in Matlab (version R2012B) and executed on a desktop computer (Intel(R) Core(TM) i72600 CPU@3.40GHz, 6Gb RAM) running Windows Home Premium (64-bit).

In Section 4 it has been shown that basically all ENZ-integrals can be written in the form of Eq. (55) where the integrand is the product of an algebraic factor $a(\rho)$, a focal factor $f(\rho)$, a polynomial factor $p(\rho)$ and a Bessel function factor $b(\rho)$. The front factor $a(\rho) f(\rho)$ is written in a systematic way as an $R^{0}$ expansion avoiding the rather involved ad hoc procedures in 
earlier schemes. While there are short-cuts in particular cases, see the developments for $I_{\mathrm{BLD}}$ in Section 6, this requires use of Clebsch-Gordan coefficients to linearize the product of two $R^{0}$-polynomials that occur when the $R^{0}$-expansions of $a(\rho)$ and $f(\rho)$ are multiplied. These Clebsch-Gordan coefficients are used again for the computation of the expansion of the product $a(\rho) f(\rho) p(\rho)$ as a series involving radial polynomials of azimuthal order that match the order of the Bessel function factor $b(\rho)$. In Section 5 we have presented a fast, recursive scheme for the computation of the required linearization coefficients, observed non-negativity and boundedness of these coefficients, and we have determined the sets outside which the coefficients vanish. Finally, the remaining integrals can be evaluated using the integral result of the classical NijboerZernike theory, for which a separate effort has been made in Section 9 for the single case of $I_{\mathrm{VMML}}$ with $|j|=2$ and --sign choice.

The new systematic approach to evaluate the ENZ-integrals allows a modular implementation, to be discussed in Subsection 10.1, due to the separation of the various dependencies. Furthermore, the new approach has the benefit that the tradeoff issue between computation time and accuracy is much better understood than in earlier schemes. In the latter schemes, certain levels of accuracy were not even practicable in terms of realistic computation times. We shall look into more detail at these issues in Subsecstions 10.2-10.3.

\subsection{Modular implementation}

In the (E)NZ approach to compute diffraction integrals $U(r, \phi ; f)$ of the type as occurs in Eq. (3), the pupil function $P(\rho, \theta)$ is thought to be expanded into a Zernike series. This yields a decoupling of the integral over the pupil disk as a product of an integral over the radial variable $\rho$ and an integral over the azimuthal variable $\theta$, in which the latter integral is readily evaluated as a Bessel function. As a result, the dependencies of $U(r, \phi ; f)$ on $(r, f)$ on one hand and on $\phi$ on the other are completely decoupled. The principal effort in the present paper is to compute the dependencies on $(r, f)$ per Zernike term, and this leads to consideration of the various $I$-integrals. In these I-integrals, see Eq. (55), the dependencies on the system parameters $s$, focal value $f$ and radial value $r$ is almost entirely separated according to the factors $a(\rho)$, $f(\rho)$ and $b(\rho)$, respectively, while the factor $p(\rho)$ comprises the degree and order of the considered circle polynomial as well as the integer $j,|j| \leq 2$, accounting for the components of the vector field and the state of polarization. This leads to a series representation in Eq. (59) of the $I$-integral in which the various dependencies are separated accordingly. In earlier schemes, there was a stronger coupling between system parameters and focal values and radial values.

\subsection{Computation time comparison}

The use of more advanced mathematical tools in the computation of the ENZ-diffraction integrals is beneficial for the tradeoff between accuracy and computation time. In Figure 4 we show this for the computation of the $I_{B}$-integral in Eq. (24) of Subsection 3.1. The three methods compared are the power-
Bessel series representation of $I_{B}$ in Eq. (16), the Bessel-Bessel series in Eq. (61) of Section 5 using an explicit Zernike representation of the focal factor and Clebsch-Gordan coefficients, and the Bessel-Bessel series of [25], Subsec. 2.2. The latter method is mathematically equivalent with the Bessel-Bessel method of Section 5, but the linearization of products $R_{2 k}^{0} R_{n}^{m}$ is done using an ad hoc method involving finite double series expressions for the coefficients, containing products of 3 factors each of which is a quotient comprising 3 or 4 binomials. In each of the three representations of $I_{B}$, we have a double series of terms of the form $F_{k}(f) R_{h}(r)$ with $k=0,1, \cdots$ and $h \in S(k)$, where $S(k)$ is a finite set of non-negative integers ( $F, R$ and $S$ depend on the representation used). The meaning of $K=0,1, \cdots$ on the horizontal axis in Figure 4 is as follows: for each representation of $I_{B}$ we include all terms $(k, h)$ with $k=0,1, \cdots, K$ and $h \in S(k)$. The standard precision in Matlab is given as 15 figures, while we have used as a reference the result of a numerical method in Maple with an accuracy requirement of 20 significant figures. The chosen values of $r$ and $f$ are $r=1, f=2 \pi$ throughout, and the degrees $n$ and azimuthal orders $m$ of the circle polynomials $Z_{n}^{m}$ are taken $(n, m)=(0,0),(4,0),(5,5),(16,4)$. It is seen that the power-Bessel series suffers from loss-of-digits and slow convergence; these effects worsen when $f$ is increased further, with $f=30$ being the limit beyond which no practical accuracies can be achieved anymore. The two Bessel-Bessel series representations are comparable with regard to accuracy, but the ad hoc method of [25], Subsec. 2.2 gets in serious trouble when degree and orders are increased further, say, beyond 40 . Furthermore, the computation time of the ad hoc Bessel-Bessel series method are much larger, especially when degree $n$ and order $m$ get large, compared with the advanced Bessel-Bessel series method using Clebsch-Gordan coefficients.

\subsection{Assessment computational load in advanced scheme}

To assess the computational load of the new advanced scheme in the various situations, we consider the series expression in Eq. (59) for the general I-integral in Eq. (55). The dependence on the system parameters $s$ and the $f$-dependence are entirely contained in the coefficients $w_{t}$ being the $R^{0}$-coefficients of the front factor $a(\rho) f(\rho)$. The $r$-dependence is entirely contained in the Jinc function $J_{s+1}(2 \pi r) / 2 \pi r$, where the summation range of $s$ is limited to $h, h \pm 1, h \pm 2$ depending on the value of $j$. The $A$-coefficients occurring in Eq. (59) are all bounded between 0 and 1 , see Section $5, b$, and the set of indices for which they are non-vanishing is indicated in Figure 3. Accordingly, one can consider in Eq. (59) summation variables $s, h, t$ that are limited to the ranges $s=h, h \pm 1, h \pm 2$ with integer $t, h \geq 0$ such that $\frac{1}{2}|h-n| \leq t \leq \frac{1}{2}(h+n)$ and $n$ the degree of the considered circle polynomial. The set of $(h, t)$ to be considered is roughly a diagonal-like set around $h=2 t \geq 0$ of width $n$. Therefore, the convergence behaviour of the triple series in Eq. (59) is chiefly determined by the decay behaviour of the Jinc function $J_{h+1}(2 \pi r) / 2 \pi r$ as $h \rightarrow \infty$ and decay behaviour of the coefficients $w_{t}$ of the front factor as $t \rightarrow \infty$.

The decay of the Jinc function $J_{h+1}(2 \pi r) / 2 \pi r$ as $h \rightarrow \infty$ takes place according to Section 5, g. In Figure 5 this decay behaviour is shown for $r=0.1,1$ and 10, respectively. It is 

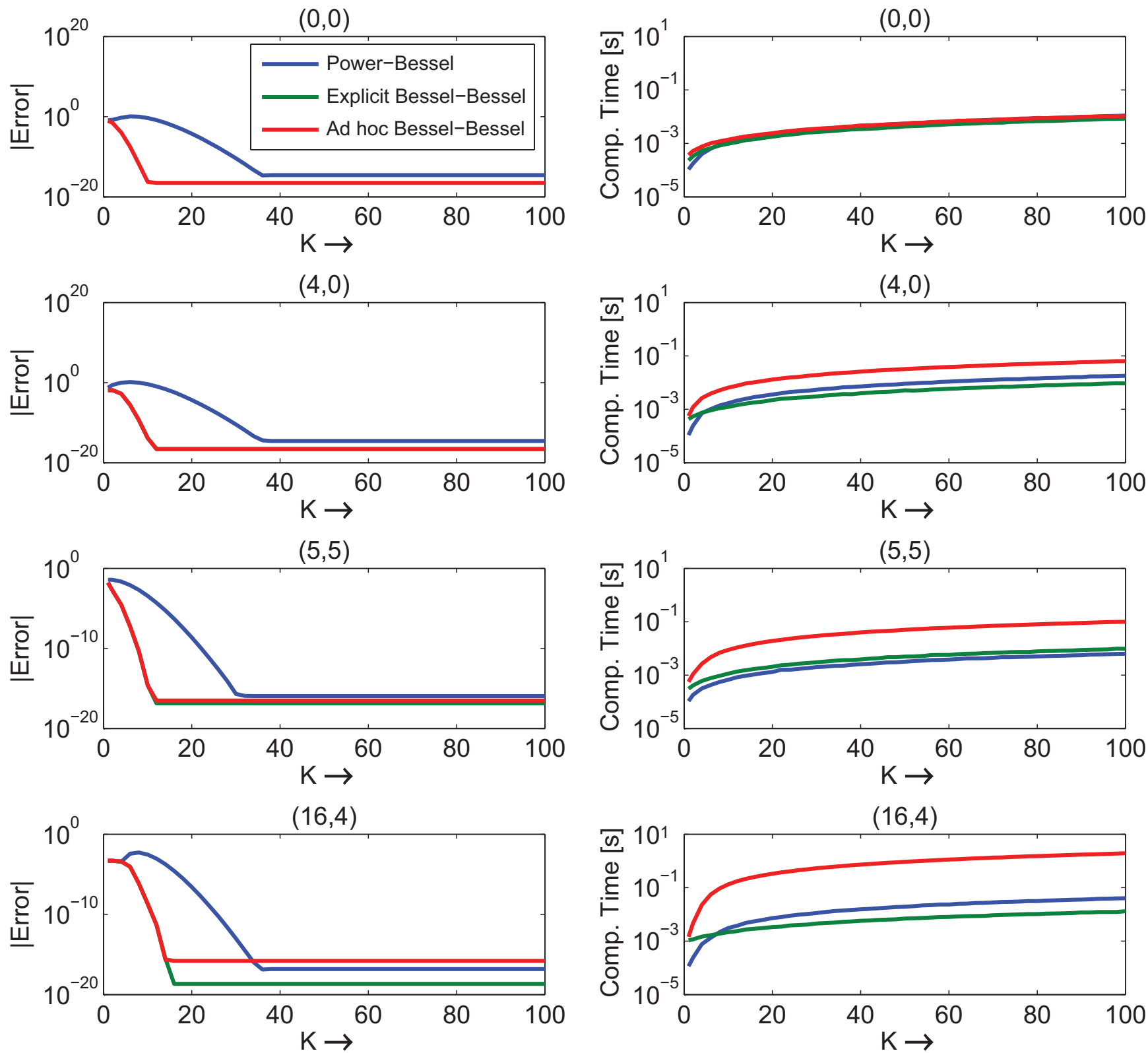

FIG. 4 Accuracy and computation time comparison for the $I_{B}$-integral in Eq. (24) of Subsec. 3.1. Blue: power-Bessel series in Eq. (16). Green: Bessel-Bessel series in Eq. (61) using Clebsch-Gordan coefficients and explicit Zernike representation of focal factor. Red: Bessel-Bessel series of [25], Subsec. 2.2 with ad hoc procedure to linearize products of circle polynomials. Throughout $r=1, f=2 \pi$ and $(n, m)=(0,0),(4,0),(5,5),(16,4)$, and the number $K$ on the horizontal axis refers to the total number of terms used after truncation of the various infinite series representations of $I_{B}$.

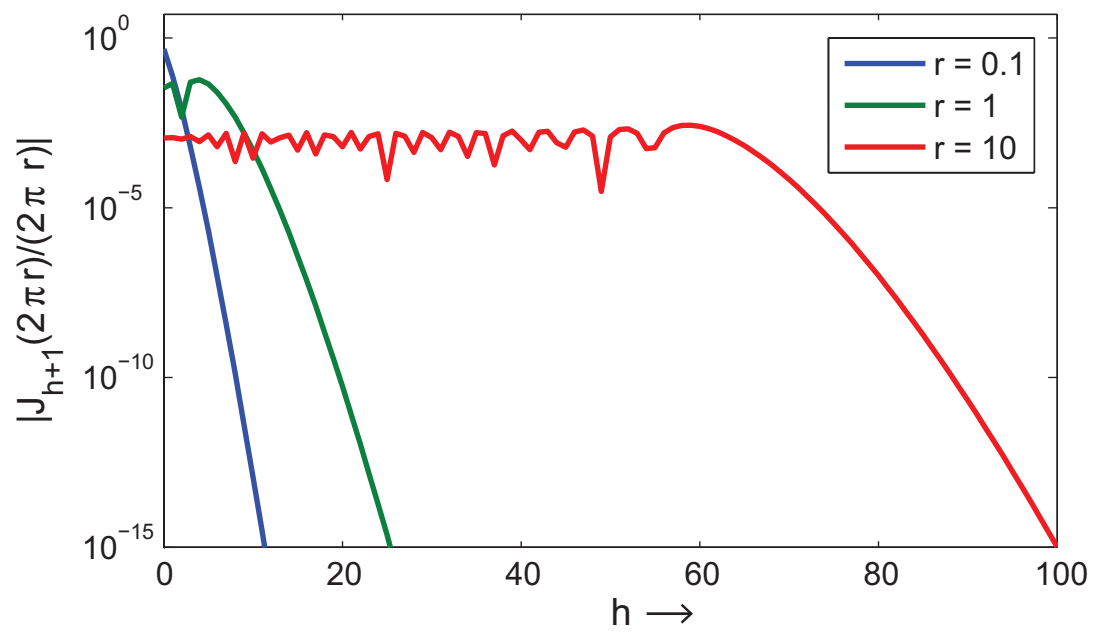

FIG. 5 Decay of the Jinc function $J_{h+1}(2 \pi r) / 2 \pi r$ as a function of $h=0,1, \cdots$ for $r=0.1,1,10$. 

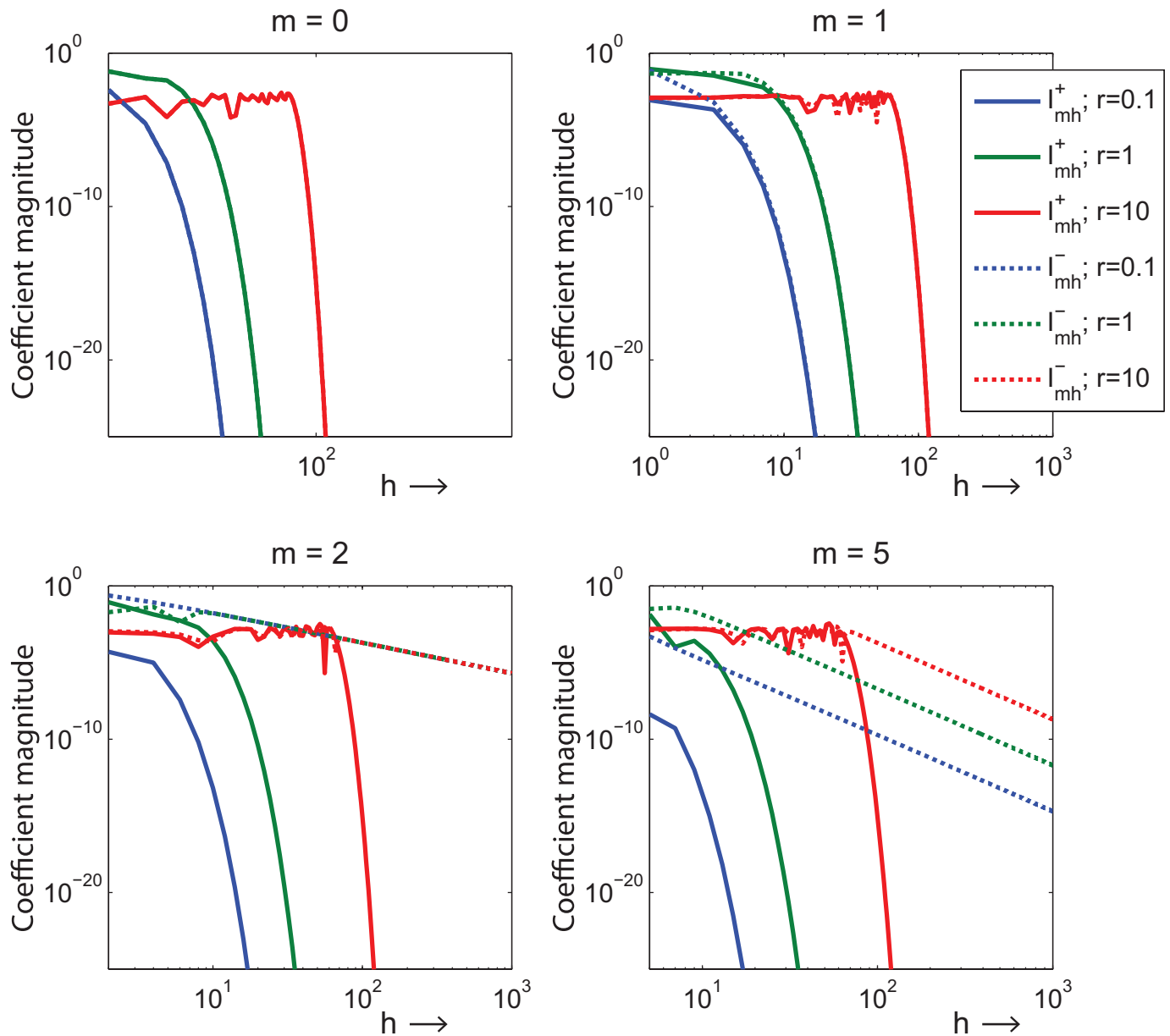

FIG. 6 Decay of the integrals $I_{m h}^{ \pm}$as function of $h=0,1, \cdots$ for the cases that $m=0,1,2,5$ and $r=0.1,1,10$.

seen that $\left|J_{h+1}(2 \pi r) / 2 \pi r\right|$ is non-negligible until $h \approx 2 \pi r$ after which ever faster decay towards 0 occurs. The nearly constant level of $\left|J_{h+1}(2 \pi r) / 2 \pi r\right|$ until $h=2 \pi r$ can be understood from Debye's asymptotic formula for Bessel functions as given in [23], 10.19. The computation time required for evaluating $J_{h+1}(2 \pi r)$ is a fraction of the time required to evaluate the Zernike expansion of the $f$-dependent front factor of the I-integrals. A similar observation holds for the evaluation of the integrals $I_{m h}^{ \pm}$, see Appendix $\mathrm{H}$, that need to be computed for the case that we consider $I_{V M M L}$ with $|j|=2$ and --sign choice.

In the case of $I_{\mathrm{VMML}}$ with backward propagating waves, the Jinc functions just considered have to be replaced by the integrals $I_{m h^{\prime}}^{ \pm}$see Eq. (116) and Appendix H. The computation time required for evaluating $I_{m h}^{ \pm}$exceeds that for computing the Jinc functions, but is still a fraction of the time needed to compute the $f$-dependence of the $I_{\mathrm{VMML}}$-integral by expanding front factors. The decay behaviour of $I_{m h}^{+}$as a function of $h \geq m$ hardly depends on $m$ and is not much different from the decay behaviour of the Jinc function $J_{h+1}(2 \pi r) / 2 \pi r, h \geq m$, when the same value of $r$ is used in $I_{m h}^{+}$. This can be understood from Eq. (120) that exhibits $I_{m h}^{+}$as an infinite series involving Jinc functions of orders $\geq h+1$. The decay behaviour of $I_{m h^{\prime}}^{-}$, however, strongly depends on $m$ and is vastly different from that of Jinc functions. For the low-order cases $|m|=0,1$, this is not yet apparent because of Eqs. (117)-(119) with the factor $m-1$ cancelling the whole series in Eq. (119) when $m=1$. When $|m|=2,3, \cdots$, the series in Eq. (119) is not cancelled, and this series contains the Jinc functions of orders $|m|-1,|m|+1, \cdots, h+1$. Therefore, it is very well conceivable that decay is not as fast and that it does depend on $m$. In fact, it is shown in Appendix $\mathrm{H}$ that $\left|I_{m h}^{-}\right|$is approximately given by $2(m-1)(2 \pi r)^{m-2} / h^{m}$ when $m=2,3, \cdots$ and $h \geq 2 \pi r$, yielding slow decay behaviour in $h$ especially when $m$ is small. In Figure 6 we show $\left|I_{m h}^{ \pm}\right|$as a function of $h$ for the cases that $m=0,1,2,5$ and $r=0.1,1,10$. While $\left|I_{m h}^{+}\right|$exhibits a decay behaviour that compares quite well with what one finds in Figure 5 in this respect for $\left|J_{h+1}(2 \pi r) / 2 \pi r\right|$, it is seen that such a thing holds for $\left|I_{m h}^{-}\right|$only when $m=0,1$. For $m=2$, there is only a $C h^{-2}$-decay behaviour with a $C$ independent of $r$. For $m=5$, there is a $C_{r} h^{-5}$-decay behaviour with $C_{r}=8(2 \pi r)^{3}$, and this accounts for the three parallel straight lines with distances dictated by $C_{r}$ in the region $h \geq 2 \pi r$.

We next consider the computation time and decay of the coefficients $w_{t}$ of the front factor $a(\rho) f(\rho)$. In Figure 7 we show the computation time to compute a set $w_{0}, w_{1}, \cdots w_{T}$ as a function of $T=0,1, \cdots$ (Figure $7(\mathrm{a})$ ), as well as the magnitude $\left|w_{t}\right|$ as a function of $t=0,1, \cdots$ for various values of $f$ and for various instances of the $I$-integral (Figure 7(b)-(d)). We choose here $s_{0}=0.95, s_{0, M}=0.25$ and $s_{0, h}=0.5$. It is seen from Figure 7 (a) that $\bar{I}_{V}, I_{\mathrm{VM}}$ and $I_{\mathrm{VMML}}$ require almost identical times for computation of the $w$-coefficients of their front factors, while the front factor of $I_{V}$ requires substantially less computation time. In the latter case we have exploited the closed form expressions in Eqs. (35), (81), (86) for the expansion coefficients in terms of spherical Bessel and Hankel 

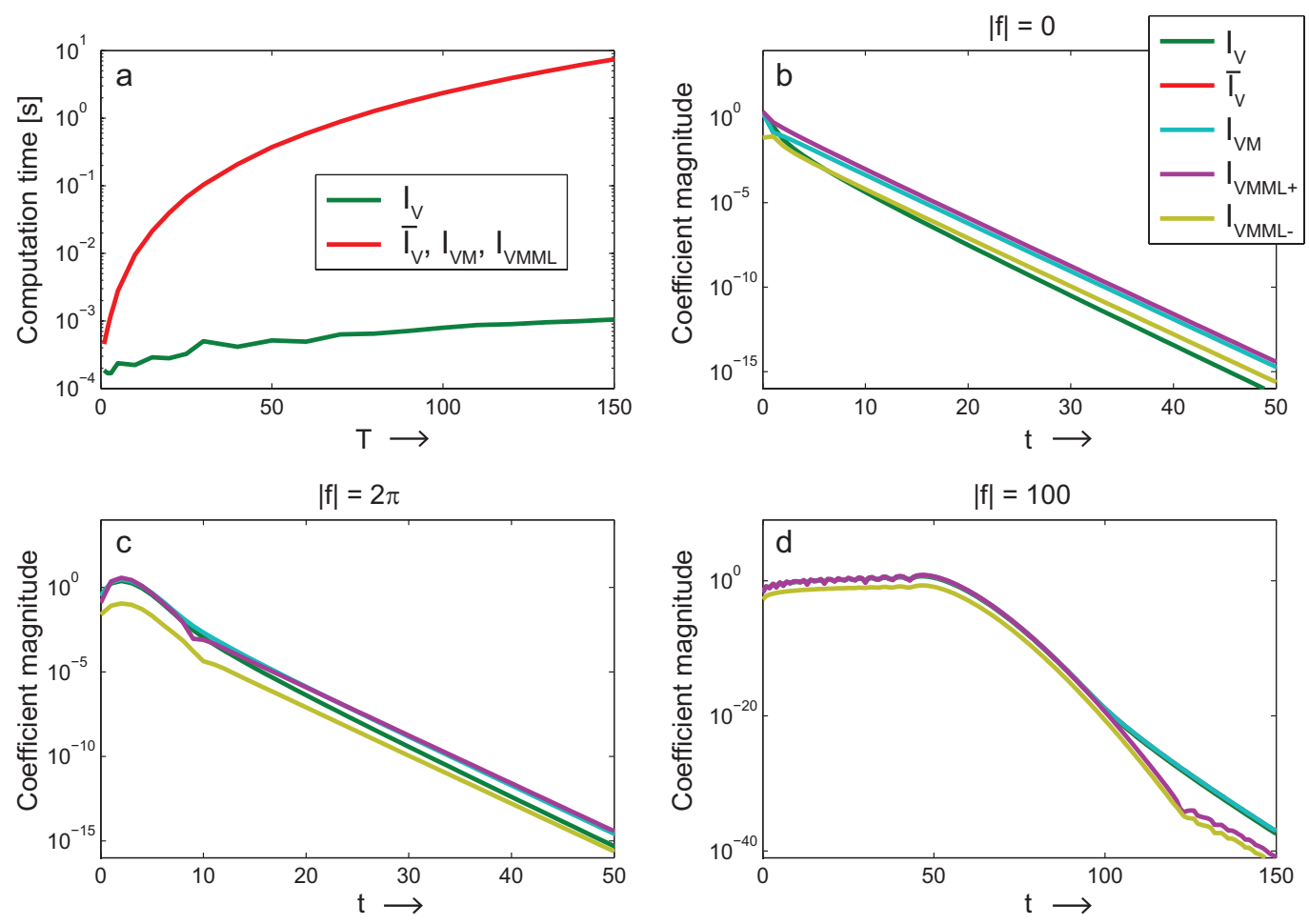

FIC. 7 Computation time and convergence of the front factor expansion coefficients as a function of $f$ for the various $I$-integrals (other parameters used: $s_{0}=0.95, s_{0, M}=0.25$ and $s_{0, h}=0.5$ ). a) the time required to compute $w_{t}, t=0,1, \cdots, T$, as a function of $T$ for a particular $I$-integral. b-d) the front factors expansion coefficients magnitude $\left|w_{t}\right|$ for $f=0,2 \pi$, and 100 , respectively.

functions. In the other cases, we have first determined the $R^{0}$-expansion of the (manipulated) algebraic factor $a(\rho)$, see Eqs. (95), Eqs. (103)-(105), Eqs. (109)-(111), and we have multiplied this $R^{0}$-expansion in Eq. (35) using Clebsch-Gordan coefficients. The Figure 7(b), (c), and (d) show the magnitude of the coefficients $w_{t}, t=0,1, \cdots$ of the various front factors for $|f|=0,2 \pi$, and 100 , respectively. It can be observed that for $|f|=0$ and $|f|=2 \pi$, the $\left|w_{t}\right|$ have decayed below $10^{-5}$ well before $t=20$. For the case that $|f|=100$, we can see that $w_{t}$ is non-negligible until $t=50$, after which rapid decay sets in. In these decay pictures, the influence of the parameter values $s$ in the algebraic factors is rather minor compared to the focus value $f$ in the focal factor. The decay behaviour is largely determined by the asymptotic behaviour of spherical Bessel and Hankel functions, see Eqs. (75)-(80). Accordingly, the $\left|w_{t}\right|$ starts to decay from the point that $t=|f| / 2$, with ever decreasing decay rate, until $t=|f| / 2 v_{0}$, after which the decay rate saturates according to Eq. (79). It should be noted that the decay of the coefficients $w_{t}$ for the case $I_{\mathrm{VMML}},|j|=2$ and backward propagating waves, slightly differs from the decay in all other cases. The reason for this is the slightly different nature of the (manipulated) algebraic function in this case where we removed a factor $\rho^{-2}$ to be cancelled by $\rho^{|j|}$, $|j|=2$.

The computational burden in the cases $\bar{I}_{V}, I_{\mathrm{VM}}$ and $I_{\mathrm{VMML}}$ as shown in Figure 7(a) can be split further into a part due to the determination of the $R^{0}$-expansion of the (manipulated) algebraic factor via the method embodied by Eqs. (37)-(44), and a part due to the determination of the $R^{0}$-expansion of the total front factor by using Clebsch-Gordan linearization coefficients for the products of two $R^{0}$-polynomials that occur when the $R^{0}$-expansion of the (manipulated) algebraic factor and the $R^{0}$-expansion of the focal factor in Eq. (35) are mul- tiplied. This is exemplified by Figure 8 showing computation times of the coefficients $w_{t}$ in

$$
\exp \left[\frac{i f}{u_{0}}\left(1-\sqrt{1-s_{0}^{2} \rho^{2}}\right)\right]=\sum_{t=0}^{\infty} w_{t} R_{2 t}^{0}(\rho),
$$

where $f=1$ and $s_{0}=0.95$. In this case, we have $w_{t}$ given explicitly by Eq. (81) in terms of spherical Bessel and Hankel functions. Alternatively, we can write

$$
\begin{aligned}
& \exp \left[\frac{\text { if }}{u_{0}}\left(1-\sqrt{1-s_{0}^{2} \rho^{2}}\right)\right]= \\
& \sqrt{1-s_{0}^{2} \rho^{2}} \frac{\exp \left[\frac{i f}{u_{0}}\left(1-\sqrt{1-s_{0}^{2} \rho^{2}}\right)\right]}{\sqrt{1-s_{0}^{2} \rho^{2}}} .
\end{aligned}
$$

The two factors at the right-hand side of Eq. (126) have explicit $R^{0}$-expansions according to Eq. (135) and Eq. (35), respectively, and then the $w_{t}$ can also be obtained from these two $R^{0}$-expansions using Clebsch-Gordan coefficients. Finally, one can also choose to find the $R^{0}$-expansion of $\sqrt{1-s_{0}^{2} \rho^{2}}$ according to the procedure in Eqs. (37)-(44), and then find the $w_{t}$ by using Clebsch-Gordan coefficients for the product in Eq. (126). This yields the three curves in Figure 8 of computation times as a function of the total number $T$ of computed coefficients $w_{t}$

- according to Eq. (81): method 1,

- according to Eqs. (135) and (35) using Clebsch-Gordan coefficients: method 2,

- according to Eqs. (37)-(44), Eq. (35) using ClebschGordan coefficients: method 3. 


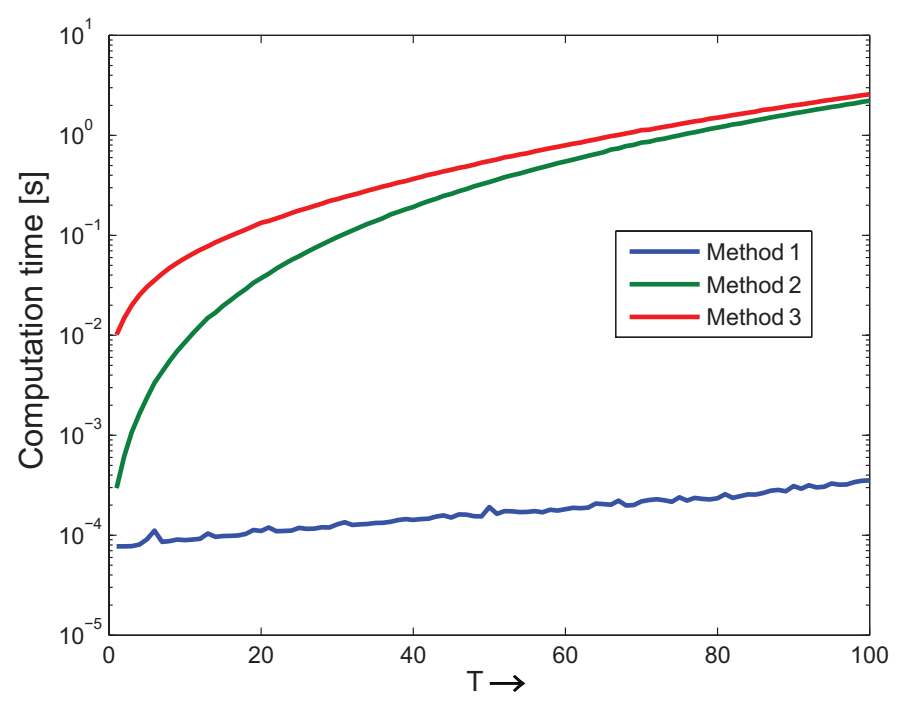

FIG. 8 Computational cost to compute the expansion coefficients $w_{t}, t=0,1, \cdots, T$ of the front factor in Eq. (125) with $f=1$ and $s_{0}=0.95$ as a function of $T=0,1, \cdots$. Blue: using explicit result Eq. (81) for the $w_{t}$ (method 1). Green: writing the front factor as product of two factors with explicitly given coefficients and using Clebsch-Gordan coefficients (method 2). Red: as method 2, except that the expansion coefficients of one of the factors is obtained by conversion of its power series into a Zernike series according to Eqs. (37)-(44) (method 3).

It is clear from Figure 8 that having an explicit form for the Zernike expansion coefficients of the front factor is very beneficial for low computation times, also when the number $T$ of computed coefficients $w_{t}$ is increased to very high levels. Furthermore, it is seen that the computational costs incurred by using Clebsch-Gordan linearization coefficients as in method 2 are modest for lower values of the number of computed coefficients. However, for moderate values of $T$, like 10 or 20, method 2 becomes $2-3$ orders more expensive than method 1 , and this trend continues towards large values of $T$. The most expensive part of using Clebsch-Gordan coefficients is the initialization in Eqs. (66)-(67) of the recursion in Eq. (68), the recursion itself being relatively cheap to use. The computational cost to determine the Zernike coefficients of $\left(1-s_{0}^{2} \rho^{2}\right)^{1 / 2}$ from its power series, as is done in method 3 , is already at the very beginning $T=1,2$ two orders higher than the cost of method 1. For large values of $T$, the curves in Figure 8 for method 2 and 3 approximate one another which indicates that at these $T$-values the computational effort is almost completely spent to the linearization procedure.

\section{CONCLUSIONS AND OUTLOOK}

We have reviewed the ENZ-theory regarding the computation of the basic integrals over the radial pupil variable that arise from the Debye approximation of the Rayleigh diffraction integral when the generalized pupil function is expanded into Zernike circle polynomials and the integration over the azimuthal pupil variable is performed analytically. We have given an account of the evolution of the form of the basic ENZ-integral as occurred in the period 2002-2010, starting from the simplest case with low NA and small defocus value to the much involved case with with high NA, vector fields and polarization with an extended object being imaged into a multi-layered focal region. The methods by which the basic ENZ-integrals were computed semi-analytically evolved accordingly, yielding in the end a not very transparent situation with certain ad hoc measures and sometimes prohibitively slow codes.

In this paper, we have introduced in the ENZ-domain more advanced analytical tools, allowing a systematic, modular computation of the basic ENZ-integral, with a much better trade-off between accuracy and computation time of the new semi-analytic expressions while also a more transparent truncation error analysis emerged. The new semi-analytic expression for the basic ENZ-integrals has the form

$$
\sum_{h, t} K(n, m, t, h) w_{t}(f, \mathrm{OS}) B_{h}(r),
$$

where $f$ is the defocus parameter and $r \geq 0$ is the radial variable in the image plane. The radial dependence is contained in $B_{h}$ and can be expressed in terms of Jinc functions. Furthermore, $w_{t}(f, \mathrm{OS})$ are the Zernike coefficients of a focal factor multiplied by an algebraic function determined by the parameters of the optical system (OS) such as NA and refractive indices. The Zernike coefficients $w_{t}(f, \mathrm{OS})$ are obtained from the Zernike coefficients of the focal factor and those of the algebraic function by using to Clebsch-Gordan related linearization coefficients. One more such a linearization step is needed to take into account the degree $n$ and the azimuthal order $m$ of the radial part $R_{n}^{|m|}$ of the considered circle polynomial. All these linearization constants are gathered in the coefficients $K(n, m, t, h)$. The Zernike coefficients of the focal factor have been obtained explicitly in terms of spherical Bessel and Hankel functions, while those of the algebraic factor can be computed from its power series expansion coefficients, the latter being computable recursively.

The computational effort required for capturing the radial dependence of the basic integrals is low compared to what is needed for the focal dependence. For all cases considered, except the case of backward propagating waves in a multilayered focal region, the decay of $B_{h}(r)$ with increasing order $h$ follows a simple law according to which contributions to the basic integral become negligible soon after $h$ has exceeded $2 \pi r$. In the exceptional case of backward propagating waves, the decay is slower and depends on the azimuthal order of the considered circle polynomials.

The computational requirements for the $f$-dependence of the basic integral is relatively large. The main efforts go into the determination of the Zernike expansion of the algebraic factor and the computation of the Zernike coefficients of the focal factor times algebraic factor using to ClebschGordan related linearization coefficients. The decay behaviour of the coefficients $w_{t}(f, \mathrm{OS})$ is for larger values of $f$ determined almost completely by the decay of the Zernike coefficients $v_{k}$ of the focal factor. These $v_{k}$ start to decay rapidly from $k=\frac{1}{2}|f|$ onwards and have an eventual decay rate $\left(1-\sqrt{1-s_{0}^{2}}\right) /\left(1+\sqrt{1-s_{0}^{2}}\right)$ with $s_{0}$ the numerical aperture of the optical system.

Finally, the required linearization coefficients and also the $K(n, m, t, h)$ are bounded in modulus by 1 . Also, the 
$K(n, m, t, h)$ are non-vanishing only for non-negative integers $h$ and $t$ such that $h$ has the same parity as $n$ and $h \geq|m|$ while $\frac{1}{2}|h-n| \leq t \leq \frac{1}{2}(h+n)$. Hence, the actual summation in Eq. (127) takes place over a set of non-negative integers $h$ and $t$ for which $(h, 2 t)$ is a diagonal-like subset of the positive quadrant of width $2 n$. This information, together with the information about decay of $B_{h}(r)$ and $w_{t}(f, \mathrm{OS})$, renders the truncation error analysis for the double series in Eq. (127) both transparent and favourable.

The modular form for the basic ENZ-diffraction integral of the double series expression in Eq. (127), with constituent terms in which dependencies are separated conveniently, is very appropriate for use in, for instance, advanced lithographic simulations. The favourable decay properties of the terms in the double series allow higher accuracy demands than the ones encountered in more conventional optical engineering. Furthermore, due to the decay properties of $w_{t}$ and $B_{h}$ and the boundedness and support properties of $K(n, m, t, h)$, one has a means to assess where a particular pupil expansion term $Z_{n}^{m}$ manifests itself in the focal range. This is potentially useful for retrieval purposes since one gains insight as to which Zernike terms can be retrieved reliably from measurements in a particular focal range.

We finally discuss extension of the new advanced approach of computing diffraction integrals to even more general optical settings. One such extension is to allow in the setting of Subsection 3.5 general complex refractive indices $n_{h}$, so that absorption and evanescent waves come into the picture. It appears to be feasible to work out this extension in the ENZdomain, but the developments for this are still too much at a research stage to further report on this here. A second extension would be that of imaging into a region with a general, rather than a stepwise, varying refractive index $n_{h}$. At present, we do not have any idea how to deal with the most general case of refractive indices that both vary in the focal and in radial and azimuthal direction.

\section{ACKNOWLEDGEMENTS}

The authors wish to thank D. Asoubar who worked out the suggestion of one of the authors (A. J. E. M. J.) to treat the diffraction integral leading to $I_{\mathrm{BLD}}$ using the explicit Zernike expansion of the focal factor and Clebsch-Gordan coefficients for the linearization of products of circle polynomials, see D. Asoubar, Nijboer-Zernike decomposition technique and its application to free-space propagation, preliminary report TUDelft. Furthermore, the authors wish to thank B. Janssen for verifying Tango's result in Eq. (140) for all combinations of products of two circle polynomials of degree $\leq 20$.

\section{A PROOF OF EQ. 35}

We use [27], (12)

$$
\begin{aligned}
& \frac{\exp \left[-i \lambda\left(T^{2}+x \rho^{2}\right)^{1 / 2}\right]}{-i \lambda\left(T^{2}+x \rho^{2}\right)^{1 / 2}}= \\
& \quad \sum_{k=0}^{\infty}(2 k+1)(-1)^{k} j_{k}\left(\lambda T_{-}\right) h_{k}^{(2)}\left(\lambda T_{+}\right) R_{2 k}^{0}(\rho),
\end{aligned}
$$

where $x$ is real, $T>0, \lambda$ complex and $T^{2}+x>0$, and

$$
T_{ \pm}=\frac{1}{2}\left[\left(T^{2}+x\right)^{1 / 2} \pm T\right]
$$

Taking $\lambda=f s_{0} / u_{0}, T=1 / s_{0}$ and $x=-1$, we get

$$
\begin{aligned}
& \frac{\exp \left[\frac{i f}{u_{0}}\left(1-\sqrt{1-s_{0}^{2} \rho^{2}}\right)\right]}{\sqrt{1-s_{0}^{2} \rho^{2}}}= \\
& \frac{f}{i u_{0}} \exp \left[\frac{i f}{u_{0}}\right] \frac{\exp \left[\frac{-i f s_{0}}{u_{0}}\left(\frac{1}{s_{0}^{2}}-\rho^{2}\right)^{1 / 2}\right]}{\frac{-i f s_{0}}{u_{0}}\left(\frac{1}{s_{0}^{2}}-\rho^{2}\right)^{1 / 2}}= \\
& \frac{f}{i u_{0}} \exp \left[\frac{i f}{u_{0}}\right] \sum_{k=0}^{\infty}(2 k+1)(-1)^{k} j_{k}\left(f s_{0} T_{-} / u_{0}\right) . \\
& h_{k}^{(2)}\left(f s_{0} T_{+} / u_{0}\right) R_{2 k}^{0}(\rho),
\end{aligned}
$$

where

$$
T_{ \pm}=\frac{1}{2}\left[\left(\frac{1}{s_{0}^{2}}-1\right)^{1 / 2} \pm \frac{1}{s_{0}}\right]=\frac{1}{2 s_{0}}\left[\left(1-s_{0}^{2}\right)^{1 / 2} \pm 1\right]
$$

We compute, using $u_{0}=1-\left(1-s_{0}^{2}\right)^{1 / 2}$,

$$
\begin{aligned}
& f s_{0} T_{-} / u_{0}=\frac{f s_{0}}{u_{0}} \cdot \frac{-1}{2 s_{0}}\left(1-\left(1-s_{0}^{2}\right)^{1 / 2}\right)=-\frac{1}{2} f \\
& f s_{0} T_{+} / u_{0}=\frac{f s_{0}}{u_{0}} \cdot \frac{1}{2 s_{0}}\left(1+\left(1-s_{0}^{2}\right)^{1 / 2}\right)=\frac{1}{2} f / v_{0}
\end{aligned}
$$

with $v_{0}$ given in Eq. (36). Finally, $j_{k}(-z)=(-1)^{k} j_{k}(z)$ and the proof is complete.

For the case that $f=0$, we have by [12], Appendix B

$$
\frac{1}{\sqrt{1-s_{0}^{2} \rho^{2}}}=\frac{2}{1+\sqrt{1-s_{0}^{2}}} \sum_{k=0}^{\infty} v_{0}^{k} R_{2 k}^{0}(\rho),
$$

and

$$
\sqrt{1-s_{0}^{2} \rho^{2}}=-\frac{1}{2} u_{0} \sum_{k=0}^{\infty}\left(\frac{v_{0}^{k-1}}{2 k-1}-\frac{v_{0}^{k+1}}{2 k+3}\right) R_{2 k}^{0}(\rho) .
$$

We shall show that Bauer's formula Eq. (34) follows from Eq. (35) upon taking $s_{0} \rightarrow 0$. With $u_{0}=1-\left(1-s_{0}^{2}\right)^{1 / 2}$, it is easy to see that the left-hand side of Eq. (35) tends to $\exp \left[\right.$ if $\left.\rho^{2}\right]$ as $s_{0} \rightarrow 0$. From [23], 10.52.4 we have that $h_{k}^{(2)}(z) \sim$ $i^{n+1} z^{-1} \exp [-i z], z \rightarrow \infty$, and so, as $s_{0} \rightarrow 0$

$$
\begin{aligned}
& \frac{\exp \left[i f / u_{0}\right]}{i u_{0}} f h_{k}^{(2)}\left(f / 2 v_{0}\right) \sim \\
& i^{k} \frac{2 v_{0}}{u_{0}} \exp \left[i f\left(\frac{1}{u_{0}}-\frac{1}{2 v_{0}}\right)\right] .
\end{aligned}
$$

The right-hand side of Eq. (136) tends to $i^{k} \exp \left[\frac{1}{2} i f\right]$ as $s_{0} \rightarrow 0$ since $2 v_{0} / u_{0} \rightarrow 1$ as $s_{0} \rightarrow 0$ while $1 / u_{0}-1 /\left(2 v_{0}\right)=1 / 2$. Hence the terms at the right-hand side of Eq. (35) tend to the terms at the right-hand side of Eq. (34), and we get Bauer's formula. 


\section{B ZERNIKE EXPANSION OF}

$$
\left(1-a \rho^{2}\right)^{\alpha}\left(1-b \rho^{2}\right)^{\beta}\left(1-c \rho^{2}\right)^{\gamma}
$$

We start by showing that the power series coefficients $q_{n}$ of $\left(1-a \rho^{2}\right)^{\alpha}\left(1-b \rho^{2}\right)^{\beta}\left(1-c \rho^{2}\right)^{\gamma}$ are given by the recursion in Eqs. (38)-(39). Letting $x=\rho^{2}$, we differentiate the identity

$$
(1-a x)^{\alpha}(1-b x)^{\beta}(1-c x)^{\gamma}=\sum_{n=0}^{\infty} q_{n} x^{n},
$$

and we obtain after multiplication by $(1-a x)(1-b x)(1-c x)$ and some rearrangements

$$
\begin{aligned}
& \sum_{n=0}^{\infty}[-(a \alpha+b \beta+c \gamma) \\
& +(a \alpha(b+c)+b \beta(a+c)+c \gamma(a+b)) x \\
& \left.-(a \alpha b c+b \beta a c+c \gamma a b) x^{2}\right] q_{n} x^{n}= \\
& \sum_{n=0}^{\infty}\left(1-(a+b+c) x+(a b+a c+b c) x^{2}-a b c x^{3}\right) . \\
& (n+1) q_{n+1} x^{n} .
\end{aligned}
$$

Comparing coefficients of equal powers of $x$ at either side of Eq. (138) gives the recursive relation Eq. (39), where the initialization $q_{-2}=q_{-1}=0, q_{0}=1$ in Eq. (38) renders Eq. (39) to be valid for $n=0,1,2$ as well.

The power series $\sum_{n} q_{n} \rho^{2 n}$ can be converted into an $R^{0}$-series $\sum_{l} u_{l} R_{2 l}^{0}$ according to Eqs. (40)-(41) when $|a|<1,|b|<1$, $|c|<1$ (which implies that $q_{n} \rightarrow 0$ exponentially fast). To prove this, we use the result

$$
\rho^{2 n}=\sum_{l=0}^{n} b_{n}(l) R_{2 l}^{0}(\rho), \quad n=0,1, \ldots,
$$

see [4], (10) (case $m=0$ ), insert Eq. (139) in $\sum_{n} q_{n} \rho^{2 n}$ and change the order of summation.

The procedure of finding the $R^{0}$-expansion $\sum_{l} u_{l} R_{2 l}^{0}$ of $\left(1-a \rho^{2}\right)^{\alpha}\left(1-b \rho^{2}\right)^{\beta}\left(1-c \rho^{2}\right)^{\gamma}$ via the power series $\sum_{n} q_{n} \rho^{2 n}$ is computationally rather expensive. This is reflected by the fact that $q$-coefficients are much larger in modulus than the $u$-coefficients (up to a factor $4^{n}$ according to [27], Appendix A). Hence, while the $R^{0}$-representation $\sum_{l} u_{l} R_{2 l}^{0}$ seems quite efficient, the computation of the coefficients $u_{l}$ uses the relatively large power series coefficients $q_{n}$.

\section{LINEARIZATION OF PRODUCTS OF RADIAL POLYNOMIALS}

We consider the problem of linearizing the product $R_{2 k}^{0} R_{n}^{|m|}$ of radial polynomials with $k=0,1, \ldots$ and integer $n$ and $m$ such that $n-|m|$ is even and non-negative. Let $n_{1}, n_{2}, m_{1}, m_{2}$ be integers such that $n_{1}-\left|m_{1}\right|$ and $n_{2}-\left|m_{2}\right|$ are even and non-negative. Then according to a result of Tango in [28] (Eq. (22-23)), we have

$$
Z_{n_{1}}^{m_{1}}(\rho, \vartheta) Z_{n_{2}}^{m_{2}}(\rho, \vartheta)=\sum_{n_{3}} A_{n_{1} n_{2} n_{3}}^{m_{1} m_{2} m_{3}} Z_{n_{3}}^{m_{3}}(\rho, \vartheta),
$$

where the Z's are general circle polynomials, see Eq. (12), and the summation is over all non-negative integers $n_{3}$ with same parity as $m_{3}=m_{1}+m_{2}$ such that

$$
\max \left(\left|m_{3}\right|,\left|n_{1}-n_{2}\right|\right) \leq n_{3} \leq n_{1}+n_{2} .
$$

The $A^{\prime}$ s are given as

$$
A_{n_{1} n_{2} n_{3}}^{m_{1} m_{2} m_{3}}=\left|C_{\frac{1}{2} m_{1}, \frac{1}{2} m_{2}, \frac{1}{2} m_{3}}^{\frac{1}{2} n_{1} \frac{1}{2} n_{2}, \frac{1}{2} n_{3}}\right|^{2}
$$

where the C's are Clebsch-Gordan coefficients that we write in the notation of [23], Ch. 34 as

$$
\begin{aligned}
& C_{\frac{1}{2} m_{1}, \frac{1}{2} m_{2}, \frac{1}{2} m_{3}}^{\frac{1}{2} n_{1}, \frac{1}{2} n_{2} \frac{1}{2} n_{3}}= \\
& \quad \sqrt{n_{3}+1}(-1)^{\frac{1}{2}\left(n_{1}-n_{2}-m_{3}\right)}\left(\begin{array}{ccc}
\frac{1}{2} n_{1} & \frac{1}{2} n_{2} & \frac{1}{2} n_{3} \\
\frac{1}{2} m_{1} & \frac{1}{2} m_{2} & -\frac{1}{2} m_{3}
\end{array}\right) .
\end{aligned}
$$

Thus for the products $R_{2 k}^{0} R_{n}^{|m|}$ of radial polynomials we have

$$
R_{2 k}^{0} R_{n}^{|m|}=\sum_{h} A_{2 k, n, h}^{0 m m} R_{h}^{|m|}
$$

where the summation range consists of all integer $h$ of same parity as $m$ that satisfy

$$
\max (|m|,|n-2 k|) \leq h \leq n+2 k .
$$

For our purposes it is of interest to have a convenient and efficient way of computing the $A^{\prime}$ 's in Eq. (144), where we assume that $m \geq 0$. The general $3 j$-symbol

$$
\left(\begin{array}{ccc}
j_{1} & j_{2} & j_{3} \\
m_{1} & m_{2} & m_{3}
\end{array}\right)
$$

as occurs in [23], Chap. 34, is non-vanishing only when $j_{1}, j_{2}, j_{3}$ are non-negative integers or half-integers such that $j_{1}+j_{2}+j_{3}$ is integer while satisfying the triangle conditions

$$
\left|j_{r}-j_{s}\right| \leq j_{t} \leq j_{r}+j_{S}
$$

for any permutation $r, s, t$ of $1,2,3$, and when

$$
m_{r}=-j_{r},-j_{r}+1, \ldots, j_{r}-1, j_{r}, \quad r=1,2,3,
$$

with

$$
m_{1}+m_{2}+m_{3}=0 .
$$

By using [23], 34.3.15 for

$$
\left(\begin{array}{ccc}
j_{1}=k & j_{2}=\frac{1}{2} n & j_{3}=\frac{1}{2} h \\
m_{1}=0 & m_{2}=\frac{1}{2} m & m_{3}=-\frac{1}{2} m
\end{array}\right),
$$

we find

$$
\begin{aligned}
& m(2 k+1)\left(\begin{array}{ccc}
k & \frac{1}{2} n & \frac{1}{2} h \\
0 & \frac{1}{2} m & -\frac{1}{2} m
\end{array}\right)=\left(k^{2}-\left(\frac{n-h}{2}\right)^{2}\right)^{1 / 2} . \\
& \left(\left(\frac{n+h+2}{2}\right)^{2}-k^{2}\right)^{1 / 2}\left(\begin{array}{ccc}
k-1 & \frac{1}{2} n & \frac{1}{2} h \\
0 & \frac{1}{2} m & -\frac{1}{2} m
\end{array}\right) \\
& +\left((k+1)^{2}-\left(\frac{n-h}{2}\right)^{2}\right)^{1 / 2} . \\
& \left(\left(\frac{n+h+2}{2}\right)^{2}-(k+1)^{2}\right)^{1 / 2}\left(\begin{array}{ccc}
k+1 & \frac{1}{2} n & \frac{1}{2} h \\
0 & \frac{1}{2} m & -\frac{1}{2} m
\end{array}\right),
\end{aligned}
$$


provided that all triangle conditions Eq. (147) are satisfied by all 3j-symbols involved in Eq. (151). Thus Eq. (151) can be used to express

$$
A_{2 k+2, n, h}^{0 m m} \text { in terms of } A_{2 k-2, n, h}^{0 m m}, A_{2 k, n, h}^{0 m m}
$$

with fixed $m, n$ and $h$ such that $m \geq 0$ and $n-m$ and $h-m$ are even and non-negative. We have, see Eq. (145), that $A$ is non-vanishing only when

$$
\frac{1}{2}|n-h| \leq k \leq \frac{1}{2}(n+h) .
$$

Hence, when we can find $A$ with $k=\frac{1}{2}|n-h|$ and $k=\frac{1}{2} \mid n-$ $h \mid+1$, we can compute all required $A^{\prime}$ s recursively in $k$ for given $m, n$ and $h$ using Eq. (151) for $k=\frac{1}{2}|n-h|+1, \frac{1}{2} \mid n-$ $h \mid+2, \ldots, \frac{1}{2}(n+h)-1$.

For computing the $A^{\prime}$ s with $k=\frac{1}{2}|n-h|$ and $k=\frac{1}{2}|n-h|+1$, we use [23], 34.2.4. We distinguish between the cases $m \leq h \leq n$ and $h \geq n$.

Case $m \leq h \leq n$. When $k=\frac{1}{2}(n-h)$, only the term with $s=k$ contributes to the series over $s$ in [23], 32.2.4, and evaluating the $\Delta$ in [23], 32.2.5, we get

$$
\begin{gathered}
\left(\begin{array}{ccc}
\frac{1}{2}(n-h) & \frac{1}{2} n & \frac{1}{2} h \\
0 & \frac{1}{2} m & -\frac{1}{2} m
\end{array}\right)=(-1)^{\frac{n-m}{2}}\left(\frac{(n-h) ! h !}{(n+1) !}\right)^{1 / 2} \\
\left(\frac{\left(\frac{1}{2}(n+m)\right) !\left(\frac{1}{2}(n-m)\right) !}{\left(\frac{1}{2}(h+m)\right) !\left(\frac{1}{2}(h-m)\right) !}\right)^{1 / 2} \frac{1}{\left(\frac{1}{2}(n-h)\right) !}
\end{gathered}
$$

When $k=\frac{1}{2}(n-h)+1$, only the terms with $s=k$ and $s=$ $k-1$ contribute to the series over $s$ in [23], 32.2.4. The $\Delta$ of [23], 32.2 .5 can also be evaluated and a computation then shows that

$$
\begin{aligned}
& \left(\begin{array}{ccc}
\frac{1}{2}(n-h)+1 & \frac{1}{2} n & \frac{1}{2} h \\
0 & \frac{1}{2} m & -\frac{1}{2} m
\end{array}\right)= \\
& (-1)^{\frac{n-m}{2}} m\left(\frac{(n-h+1) !(h+1) !}{(n+2) !}\right)^{1 / 2} . \\
& \quad\left(\frac{\left(\frac{1}{2}(n+m)\right) !\left(\frac{1}{2}(n-m)\right) !}{\left(\frac{1}{2}(h+m)\right) !\left(\frac{1}{2}(h-m)\right) !}\right)^{1 / 2} \frac{1}{\left(\frac{1}{2}(n-h)\right) !} .
\end{aligned}
$$

Comparing Eq. (154) and Eq. (155), it is readily seen that

$$
\begin{aligned}
& \left(\begin{array}{ccc}
\frac{1}{2}(n-h)+1 & \frac{1}{2} n & \frac{1}{2} h \\
0 & \frac{1}{2} m & -\frac{1}{2} m
\end{array}\right)= \\
& m\left(\frac{n-h+1}{(n+2) h}\right)^{1 / 2}\left(\begin{array}{ccc}
\frac{1}{2}(n-h) & \frac{1}{2} n & \frac{1}{2} h \\
0 & \frac{1}{2} m & -\frac{1}{2} m
\end{array}\right) .
\end{aligned}
$$

Inspecting the recursion in Eq. (151), it turns out that the result Eq. (156) coincides with what one finds when we would have set

$$
\left(\begin{array}{ccc}
\frac{1}{2}(n-h)-1 & \frac{1}{2} n & \frac{1}{2} h \\
0 & \frac{1}{2} m & -\frac{1}{2} m
\end{array}\right)=0
$$

in Eq. (151). Hence, when we initialize according to Eq. (154) and Eq. (157), the recursion Eq. (151) can be applied for $k=\frac{1}{2}(n-h), \frac{1}{2}\left(n-h+1, \ldots, \frac{1}{2}(n+h)-1\right.$ to find all required non-zero $A$ 's for given $m, n$ and $h$.

Case $h \geq n$. When $k=\frac{1}{2}(h-n)$, only the term with $s=0$ contributes to the series in [23], 32.2.4, and we get as in Eq. (154)

$$
\begin{gathered}
\left(\begin{array}{ccc}
\frac{1}{2}(h-n) & \frac{1}{2} n & \frac{1}{2} h \\
0 & \frac{1}{2} m & -\frac{1}{2} m
\end{array}\right)=(-1)^{\frac{1}{2}(h+m)-n}\left(\frac{(h-n) ! n !}{(h+1) !}\right)^{1 / 2} \\
\left(\frac{\left(\frac{1}{2}(h+m)\right) !\left(\frac{1}{2}(h-m)\right) !}{\left(\frac{1}{2}(n+m)\right) !\left(\frac{1}{2}(n-m)\right) !}\right)^{1 / 2} \frac{1}{\left(\frac{1}{2}(h-n)\right) !} .
\end{gathered}
$$

When $k=\frac{1}{2}(h-n)+1$, only the terms with $s=0,1$ contribute to the series in [23], 32.2.4, and we get after a computation as in Eq. (155)

$$
\begin{aligned}
& \left(\begin{array}{ccc}
\frac{1}{2}(h-n)+1 & \frac{1}{2} n & \frac{1}{2} h \\
0 & \frac{1}{2} m & -\frac{1}{2} m
\end{array}\right)= \\
& \quad(-1)^{\frac{1}{2}(h+m)-n} m\left(\frac{(h-n+1) !(n-1) !}{(h+2) !}\right)^{1 / 2} . \\
& \quad\left(\frac{\left(\frac{1}{2}(h+m)\right) !\left(\frac{1}{2}(h-m)\right) !}{\left(\frac{1}{2}(n+m)\right) !\left(\frac{1}{2}(n-m)\right) !}\right)^{1 / 2} \frac{1}{\left(\frac{1}{2}(h-n)\right) !} .
\end{aligned}
$$

Just as in Eq. (156) and Eq. (157), it appears that we would have obtained Eq. (159) using the recursion in Eq. (151) with $k=\frac{1}{2}(h-n)$ when we would have used Eq. (158) and

$$
\left(\begin{array}{ccc}
\frac{1}{2}(h-n)-1 & \frac{1}{2} n & \frac{1}{2} h \\
0 & \frac{1}{2} m & -\frac{1}{2} m
\end{array}\right)=0 .
$$

Hence, when we initialize according to Eq. (158) and Eq. (160), the recursion Eq. (151) used with $k=\frac{1}{2}(h-n), \frac{1}{2}(h-n)+$ $1, \ldots, \frac{1}{2}(n+h)-1$ yields all non-zero $A$ 's for given $m, n$ and $h$.

The expressions in Eq. (154) and Eq. (158) can be obtained from one another by interchanging $n$ and $h$. For this also observe that

$$
(-1)^{\frac{h-m}{2}}=(-1)^{\frac{1}{2}(h+m)-n}
$$

since $m, h$ and $n$ have the same parity. We conclude that all claims about computing the $A^{\prime}$ s in Sec. 5 are proved.

For the proof of the identity in Eq. (65), we note that

$$
\begin{aligned}
& R_{2 k}^{0} R_{n}^{m}=\sum_{h} 2(h+1) I_{m n h k} R_{h}^{m}, \\
& R_{h}^{m} R_{n}^{m}=\sum_{k} 2(2 k+1) I_{m n h k} R_{2 k}^{0},
\end{aligned}
$$

where

$$
\begin{aligned}
& I_{m n h k}= \\
& \quad \int_{0}^{1} R_{2 k}^{0}(\rho) R_{n}^{m}(\rho) R_{h}^{m}(\rho) \rho d \rho=\frac{1}{2(h+1)} A_{2 k, n, h}^{0 m m} .
\end{aligned}
$$

Evaluation of Eq. (162) and Eq. (163) at $\rho=1$ (where all radial polynomials have the value 1 ) then gives Eq. (65). 


\section{D $R^{|m+j|}$-EXPANSION OF $\rho^{|j|} R_{h}^{|m|}(\rho)$}

With integer $j, m$ and $n$ such that $n-|m|$ is even and non-negative, it is required to find the $R^{|m+j|}$-expansion of $\rho^{|j|} R_{n}^{|m|}(\rho)$. We use the linearization formulas Eqs. (140)-(142) with

$$
\begin{aligned}
& m_{1}=j, \quad n_{1}=|j|, \quad m_{2}=m, n_{2}=h ; \\
& n_{3}=l, \quad m_{3}=m+j
\end{aligned}
$$

so that $3 j$-symbols

$$
\left(\begin{array}{ccc}
\left|\frac{1}{2} j\right| & \frac{1}{2} h & \frac{1}{2} l \\
\frac{1}{2} j & \frac{1}{2} m & -\frac{1}{2}(m+j)
\end{array}\right)
$$

appear in Eq. (143). For these $3 j$-symbols we can use the closed form expression in [23], 34.3.7, where for the case $j \leq 0$ we first need to apply [23], 34.3.10, and this yields after some rearrangements the result Eq. (50). The low- $|j|$ special cases (51)(53) can also be found more directly by using

$$
\begin{aligned}
\rho R_{n}^{m}(\rho) & =\frac{q+1}{n+1} R_{n+1}^{m+1}(\rho)+\frac{p}{n+1} R_{n-1}^{m+1}(\rho), \\
m & =0,1, \ldots, \\
\rho R_{n}^{m}(\rho) & =\frac{p+1}{n+1} R_{n}^{m-1}(\rho)+\frac{q}{n+1} R_{n-1}^{m-1}(\rho), \\
m & =1,2, \ldots,
\end{aligned}
$$

where $p=\frac{1}{2}(n-m)$ and $q=\frac{1}{2}(n+m)$.

\section{E ORTHOGONALITY OF $C_{2 k}^{0}(f)$ AND $B_{h}(r)$}

With

$$
C_{2 k}^{0}(f)=(2 k+1) i^{k} \exp \left[\frac{1}{2} i f\right] j_{k}\left(\frac{1}{2} f\right),
$$

where $-\infty<f<\infty$, we have for $k_{1}, k_{2}=0,1, \ldots$

$$
\begin{aligned}
& \int_{-\infty}^{\infty} C_{2 k_{1}}^{0}(f) C_{2 k_{2}}^{0 *}(f) d f= \\
& \quad\left(2 k_{1}+1\right)\left(2 k_{2}+1\right) i^{k_{1}-k_{2}} \int_{-\infty}^{\infty} j_{k_{1}}\left(\frac{1}{2} f\right) j_{k_{2}}\left(\frac{1}{2} f\right) d f .
\end{aligned}
$$

Since $j_{k}(z)$ is even in $z$ when $k$ is even and odd in $z$ when $k$ is odd, the integrals in Eq. (170) vanish when $k_{1}$ and $k_{2}$ have different parity. When $k_{1}$ and $k_{2}$ have same parity, we have from [23], 10.47.3 and 10.22.55, that

$$
\begin{aligned}
& \int_{-\infty}^{\infty} j_{k_{1}}\left(\frac{1}{2} f\right) j_{k_{2}}\left(\frac{1}{2} f\right) d f=4 \int_{0}^{\infty} j_{k_{1}}(z) j_{k_{2}}(z) d z= \\
& 2 \pi \int_{0}^{\infty} \frac{1}{z} J_{k_{1}+1 / 2}(z) J_{k_{2}+1 / 2}(z) d z=\frac{\pi}{k_{1,2}+1 / 2} \delta_{k_{1} k_{2}},
\end{aligned}
$$

and this yields Eq. (69).

Next, with

$$
B_{h}(r)=\frac{J_{h+1}(2 \pi r)}{2 \pi r},
$$

where $r \geq 0$, we have for $h_{1}=|m|+2 l_{1}, h_{2}=|m|+2 l_{2}$ in which $l_{1}, l_{2}=0,1, \ldots$

$$
\begin{aligned}
& \int_{0}^{\infty} B_{h_{1}}(r) B_{h_{2}}(r) r d r= \\
& \frac{1}{4 \pi^{2}} \int_{0}^{\infty} \frac{1}{z} J_{h_{1}+1}(z) J_{h_{2}+1}(z) d z=\frac{1}{8 \pi^{2}\left(h_{1,2}+1\right)} \delta_{h_{1} h_{2},}
\end{aligned}
$$

where [23], 10.22.55 has been used. This is Eq. (70).

\section{F DERIVATIVES OF $f j_{k}(f / 2) h_{k}^{(2)}\left(f / 2 v_{0}\right)$}

We compute for $k=0,1, \ldots\left(()^{\prime}\right.$ denoting $\left.\frac{d}{d f}\right)$

$$
\left(f j_{k}(f / 2) h_{k}\left(f / 2 v_{0}\right)\right)^{\prime}=j_{k} h_{k}+\frac{1}{2} f j_{k}^{\prime} h_{k}+\frac{1}{2 v_{0}} f j_{k} h_{k}^{\prime},
$$

where $h=h^{(1)}$ or $h^{(2)}$ and where we have deleted the argument $f / 2$ from $j_{k}, j_{k}^{\prime}$ and the argument $f / 2 v_{0}$ from $h_{k}, h_{k}^{\prime}$ at the right-hand side of Eq. (174). Next, we use [23], 10.51.2,

$$
\begin{aligned}
& j_{k}^{\prime}(z)=j_{k-1}(z)-\frac{k+1}{z} j_{k}(z), \\
& h_{k}^{\prime}(z)=-h_{k+1}(z)+\frac{k}{z} h_{k}(z),
\end{aligned}
$$

where for the case $k=0$ in the first item in Eq. (175) (not covered by [23], 10.51.2) we set $j_{-1}(z)=z^{-1} \cos z$. Then we get

$$
\begin{aligned}
& \left(f j_{k}(f / 2) h_{k}\left(f / 2 v_{0}\right)\right)^{\prime}=j_{k} h_{k}+\frac{1}{2} f\left(j_{k-1}-\frac{k+1}{f / 2} j_{k}\right) h_{k} \\
& +\frac{1}{2 v_{0}} f j_{k}\left(-h_{k+1}+\frac{k}{f / 2 v_{0}} h_{k}\right)= \\
& \quad \frac{1}{2} f j_{k-1} h_{k}-\frac{1}{2 v_{0}} f j_{k} h_{k+1},
\end{aligned}
$$

and this is Eq. (82).

Next using Eq. (176) and remembering the convention of deleting arguments from $j$ and $h$, we get

$$
\begin{gathered}
\left(f j_{k}(f / 2) h_{k}\left(f / 2 v_{0}\right)\right)^{\prime \prime}=\left(\frac{1}{2} f j_{k-1} h_{k}-\frac{1}{2 v_{0}} f j_{k} h_{k+1}\right)^{\prime}= \\
\frac{1}{2} j_{k-1} h_{k}+\frac{1}{4} f j_{k-1}^{\prime} h_{k}+\frac{1}{4 v_{0}} f j_{k-1} h_{k}^{\prime} \\
\quad-\frac{1}{2 v_{0}} j_{k} h_{k+1}-\frac{1}{4 v_{0}} f j_{k}^{\prime} h_{k+1}-\frac{1}{4 v_{0}^{2}} f j_{k} h_{k+1}^{\prime} \cdot
\end{gathered}
$$

Now use, see again [23], 10.51 .2 with extension to the case $k=0$ as well as done above,

$$
\begin{aligned}
& j_{k-1}^{\prime}(z)=-j_{k}(z)+\frac{k-1}{z} j_{k-1}(z), \\
& h_{k}^{\prime}(z)=-h_{k+1}(z)+\frac{k}{z} h_{k}(z)
\end{aligned}
$$

and

$$
\begin{aligned}
& j_{k}^{\prime}(z)=j_{k-1}(z)-\frac{k+1}{z} j_{k}(z) \\
& h_{k+1}^{\prime}(z)=h_{k}(z)-\frac{k+2}{z} h_{k+1}(z)
\end{aligned}
$$


in the first and second line of the right-hand side of Eq. (177), respectively. This gives

$$
\begin{aligned}
& \left(f j_{k}(f / 2) h_{k}\left(f / 2 v_{0}\right)\right)^{\prime \prime}=\frac{1}{2} j_{k-1} h_{k}+\frac{1}{4} f\left(-j_{k}+\frac{k-1}{f / 2} j_{k-1}\right) h_{k} \\
& \quad+\frac{1}{4 v_{0}} f j_{k-1}\left(-h_{k+1}+\frac{k}{f / 2 v_{0}} h_{k}\right)-\frac{1}{2 v_{0}} j_{k} h_{k+1} \\
& \quad-\frac{1}{4 v_{0}} f\left(j_{k-1}-\frac{k+1}{f / 2} j_{k}\right) h_{k+1} \\
& \quad-\frac{1}{4 v_{0}^{2}} f j_{k}\left(h_{k}-\frac{k+2}{f / 2 v_{0}} h_{k+1}\right)= \\
& \left(k j_{k-1} h_{k}-\frac{1}{4} f j_{k} h_{k}-\frac{1}{4 v_{0}} f j_{k-1} h_{k+1}\right) \\
& \quad+\left(-\frac{1}{4 v_{0}} f j_{k-1} h_{k+1}-\frac{1}{4 v_{0}^{2}} f j_{k} h_{k}+\frac{k+1}{v_{0}} j_{k} h_{k+1}\right)=
\end{aligned}
$$$$
k j_{k-1} h_{k}-\frac{1}{4} f j_{k} h_{k}-\frac{1}{2 v_{0}} f j_{k-1} h_{k+1}-\frac{1}{4 v_{0}^{2}} f j_{k} h_{k}+
$$$$
\frac{k+1}{v_{0}} j_{k} h_{k+1}
$$

and this is Eq. (88)

We note that using $j_{-1}(z)=z^{-1} \cos z$ above may yield problems when $z=f / 2=0$. The aim of the present appendix is to have a computational result for the $R^{0}$-expansions in Eq. (35), Eq. (81), Eq. (86) and Eq. (90). In the case that $f=0$, this amounts to finding the $R^{0}$-expansions of

$$
\frac{1}{\sqrt{1-s_{0}^{2} \rho^{2}}}, 1, \sqrt{1-s_{0}^{2} \rho^{2}} \text { and } \frac{1}{1+\sqrt{1-s_{0}^{2} \rho^{2}}}
$$

respectively. The first and third case in Eq. (181) have been given in Eq. (134) and Eq. (135), respectively, the second case is trivial, and the fourth case will be done in Appendix G.
G $R^{0}$-EXPANSION OF

$$
\begin{aligned}
& \left(1+\sqrt{1-s_{0}^{2} \rho^{2}}\right)^{-1} \cdot \\
& \exp \left[\frac{i f}{u_{0}}\left(1-\sqrt{1-s_{0}^{2} \rho^{2}}\right)\right]
\end{aligned}
$$

We have

$$
\begin{aligned}
& \left(1+\sqrt{1-s_{0}^{2} \rho^{2}}\right)^{-1} \exp \left[\frac{i f}{u_{0}}\left(1-\sqrt{1-s_{0}^{2} \rho^{2}}\right)\right]= \\
& \frac{\exp \left[i f / u_{0}\right]}{s_{0}^{2} \rho^{2}}\left(1-\sqrt{1-s_{0}^{2} \rho^{2}}\right) \exp \left[-\frac{i f}{u_{0}} \sqrt{1-s_{0}^{2} \rho^{2}}\right]= \\
& \frac{\exp \left[i f / u_{0}\right]}{s_{0}^{2} \rho^{2}} . \\
& \int_{0}^{\rho} \frac{d}{d \rho_{1}}\left\{\left(1-\sqrt{1-s_{0}^{2} \rho_{1}^{2}}\right) \exp \left[-\frac{i f}{u_{0}} \sqrt{1-s_{0}^{2} \rho_{1}^{2}}\right]\right\} d \rho_{1}= \\
& \exp \left[i f / u_{0}\right] \frac{1}{\rho^{2}} \cdot \\
& \int_{0}^{\rho}\left(\frac{1+i f / u_{0}}{\sqrt{1-s_{0}^{2} \rho^{2}}}+\frac{f}{i u_{0}}\right) \exp \left[-\frac{i f}{u_{0}} \sqrt{1-s_{0}^{2} \rho_{1}^{2}}\right] \rho_{1} d \rho_{1} .
\end{aligned}
$$

Using Eq. (35) and Eq. (81), we get

$$
\begin{array}{r}
\left(1+\sqrt{1-s_{0}^{2} \rho^{2}}\right)^{-1} \exp \left[\frac{i f}{u_{0}}\left(1-\sqrt{1-s_{0}^{2} \rho^{2}}\right)\right]= \\
\frac{1}{i u_{0}} \exp \left[i f / u_{0}\right] \frac{1}{\rho^{2}} \int_{0}^{\rho} \sum_{k=0}^{\infty} d_{k} R_{2 k}^{0}\left(\rho_{1}\right) \rho_{1} d \rho_{1},
\end{array}
$$

where

$$
\begin{aligned}
& d_{k}= \\
& (2 k+1)\left\{\left(1+i f / u_{0}\right)+f \frac{d}{d f}\right\}\left[f j_{k}(f / 2) h_{k}^{(2)}\left(f / 2 v_{0}\right)\right] .
\end{aligned}
$$

We shall show below that

$$
\begin{aligned}
& \frac{1}{\rho^{2}} \int_{0}^{\rho} R_{2 k}^{0}\left(\rho_{1}\right) \rho_{1} d \rho_{1}= \\
& \frac{1}{2(k+1)}\left[R_{2 k}^{0}(\rho)+\frac{(-1)^{k}}{k} \sum_{l=0}^{k-1}(2 l+1)(-1)^{l} R_{2 l}^{0}(\rho)\right],
\end{aligned}
$$

where the series over $l$ vanishes when $k=0$. Using this in Eq. (183), we get after some rearrangements with double series

$$
\begin{aligned}
& \left(1+\sqrt{1-s_{0}^{2} \rho^{2}}\right)^{-1} \exp \left[\frac{i f}{u_{0}}\left(1-\sqrt{1-s_{0}^{2} \rho^{2}}\right)\right]= \\
& \frac{1}{i u_{0}} \exp \left[i f / u_{0}\right] \cdot \\
& \sum_{k=0}^{\infty}\left(\frac{d_{k}}{2 k+2}+\sum_{l=k+1}^{\infty}(-1)^{k-l} \frac{(2 k+1) d_{l}}{2 l(l+1)}\right) R_{2 k}^{0}(\rho),
\end{aligned}
$$

and this is Eq. (91). 
It remains to show Eq. (185). From $R_{2 k}^{0}\left(\rho_{1}\right)=P_{k}\left(2 \rho_{1}^{2}-1\right)$, with $P_{k}$ the Legendre polynomial of degree $k$, we have

$$
\frac{1}{\rho^{2}} \int_{0}^{\rho} R_{2 k}^{0}\left(\rho_{1}\right) \rho_{1} d \rho_{1}=\frac{1}{2(1+x)} \int_{-1}^{x} P_{k}\left(x_{1}\right) d x_{1},
$$

where $x=2 \rho^{2}-1$ and the substitution $x_{1}=2 \rho_{1}^{2}-1$ has been used in the integral on the left-hand side. By [29], V(10.10), we have for $k=1,2, \ldots$ (for $k=0$, the identity in Eq. (185) is a triviality since $R_{0}^{0}(\rho)=1$ and the series on the right-hand side vanishes)

$$
\int_{-1}^{x} P_{k}\left(x_{1}\right) d x_{1}=\frac{1}{2 k+1}\left(P_{k+1}(x)-P_{k-1}(x)\right),
$$

where it also has been used that

$$
P_{k+1}(-1)=P_{k-1}(-1)=(-1)^{k+1} .
$$

Next, we use the Christoffel-Darboux formula, [29], V(10.3),

$$
\begin{array}{r}
\frac{P_{k+1}(x) P_{k}(y)-P_{k}(x) P_{k+1}(y)}{x-y}= \\
\frac{1}{k+1} \sum_{l=0}^{k}(2 l+1) P_{l}(x) P_{l}(y)
\end{array}
$$

with $y=-1$, so that by Eq. (189)

$$
\frac{P_{k+1}(x)}{1+x}=-\frac{P_{k}(x)}{1+x}+\frac{(-1)^{k}}{k+1} \sum_{l=0}^{k}(2 l+1)(-1)^{l} P_{l}(x) \text {. }
$$

Using Eq. (191) with $k-1$ instead of $k$ to rewrite $-P_{k}(x) /(1+x)$ at the right-hand side of Eq. (191), we get

$$
\begin{aligned}
& \frac{P_{k+1}(x)}{1+x}=\frac{P_{k-1}(x)}{1+x}+\frac{2 k+1}{k+1} P_{k}(x) \\
& \quad+(2 k+1)(-1)^{k} \sum_{l=0}^{k-1} \frac{2 l+1}{k(k+1)}(-1)^{l} P_{l}(x) .
\end{aligned}
$$

It then follows from Eq. (188) and Eq. (192) that

$$
\begin{aligned}
& \frac{1}{2(1+x)} \int_{1}^{x} P_{k}\left(x_{1}\right) d x_{1}= \\
& \frac{1}{2(k+1)} P_{k}(x)+(-1)^{k} \sum_{l=0}^{k-1} \frac{2 l+1}{2 k(k+1)}(-1)^{l} P_{l}(x),
\end{aligned}
$$

and this is Eq. (185), see Eq. (187).

The case $f=0$ in Eq. (184), Eq. (186) is a bit awkward. For that case, we proceed as above, using now that

$$
\left(1+\sqrt{1-s_{0}^{2} \rho^{2}}\right)^{-1}=\frac{1}{\rho^{2}} \int_{0}^{\rho} \frac{\rho_{1} d \rho_{1}}{\sqrt{1-s_{0}^{2} \rho_{1}^{2}}}
$$

and the $R^{0}$-expansion in Eq. (134) of $1 / \sqrt{1-s_{0}^{2} \rho_{1}^{2}}$. There results as above

$$
\begin{aligned}
& \left(1+\sqrt{1-s_{0}^{2} \rho^{2}}\right)^{-1}=\frac{1}{1+\sqrt{1-s_{0}^{2}}} . \\
& \quad \sum_{k=0}^{\infty}\left(\frac{v_{0}^{k}}{k+1}+(2 k+1) \sum_{l=k+1}^{\infty} \frac{(-1)^{k-l} v_{0}^{l}}{l(l+1)}\right) R_{2 k}^{0}(\rho) .
\end{aligned}
$$

\section{H COMPUTATION OF $I_{m h}^{ \pm}$}

We shall compute the integrals

$$
I_{m h}^{ \pm}=\int_{0}^{1} R_{h}^{|m|}(\rho) J_{m \pm 2}(2 \pi r \rho) \rho d \rho,
$$

where $m$ and $h$ are integers with $h-|m|$ even and nonnegative.

When $m<0$, it follows from $J_{-k}(z)=(-1)^{k} J_{k}(z)$ that

$$
J_{m \pm 2}(2 \pi r \rho)=(-1)^{m} J_{-m \mp 2}(2 \pi r \rho),
$$

and this shows Eq. (117). Furthermore, for $m=0$ we use that $J_{-2}(z)=J_{2}(z)$, and this shows Eq. (118), and for $m=1$ we have

$$
I_{1 h}^{-}=-\int_{0}^{1} R_{h}^{1}(\rho) J_{1}(2 \pi r \rho) \rho d \rho=-(-1)^{\frac{h-1}{2}} \frac{J_{h+1}(2 \pi r)}{2 \pi r}
$$

by the basic result Eq. (9) of the NZ-theory.

It remains to consider

$$
I_{m h}^{+}, \quad m=0,1, \ldots ; \quad I_{m h}^{-}, \quad m=2,3, \ldots .
$$

We use the identity, due to Nijboer ([2], (2.24)),

$R_{h}^{m}=\frac{p+1}{q} R_{h}^{m-2}$

$+\frac{m-1}{q(p+1)} \sum_{k=1}^{p+1}(h-2 k+1) \frac{\left(\begin{array}{c}p+1 \\ k\end{array}\right)}{\left(\begin{array}{c}q-1 \\ k\end{array}\right)}(-1)^{k+1} R_{h-2 k}^{m-2}$,

where $m=2,3, \ldots, h=m, m+2, \ldots$ and $p=\frac{1}{2}(h-m), q=$ $\frac{1}{2}(h+m)$. Then, for such $m$ and $h$

$$
\begin{aligned}
& I_{m h}^{-}=\int_{0}^{1} R_{h}^{m}(\rho) J_{m-2}(2 \pi r \rho) \rho d \rho= \\
& -(-1)^{p} \frac{p+1}{q} \frac{J_{h+1}(2 \pi r)}{2 \pi r}+(-1)^{p} \frac{m-1}{q(p+1)} . \\
& \sum_{k=1}^{p+1}(h-2 k+1) \frac{\left(\begin{array}{c}
p+1 \\
k
\end{array}\right)}{\left(\begin{array}{c}
q-1 \\
k
\end{array}\right)} \frac{J_{h-2 k+1}(2 \pi r)}{2 \pi r}
\end{aligned}
$$

as follows from the basic result Eq. (9) of the NZ-theory. Note that Eq. (201) for the case $m=1$ would yield the result Eq. (198) due to the factor $m-1$ in front of the series over $k$ in Eq. (201).

We shall derive the following formula, with $m=2,3, \cdots$,

$$
\begin{aligned}
I_{m h}^{-}= & \frac{1}{2}\left(\frac{1}{2} v\right)^{m-2}(m-1) \frac{(-1)^{p} p !}{(m+p) !}+(-1)^{p+1} \frac{J_{m+2 p+1}(v)}{v} \\
& +(-1)^{p+1}(m-1) \int_{0}^{1} \frac{2 J_{m+2 p+1}(v w)}{v} \frac{\mathrm{d} w}{w^{m}}
\end{aligned}
$$


where $v=2 \pi r$. In view of rapid decay of $J_{v}(z)$ in $v$ when $v \geq|z|$, it follows that for $h \geq 2 \pi r$ the first term at the righthand side of Eq. (202) gives the leading order behaviour of $I_{m h}^{-}$ when $h \geq 2 \pi r$. Consequently,

$$
\left|I_{m h}^{-}\right| \approx 2(m-1)(2 \pi r)^{m-2} / h^{m}, h \geq 2 \pi r,
$$

where we have used that for large $p=\frac{1}{2}(h-m)$

$$
\begin{aligned}
& \frac{p !}{(m+p) !}=\frac{1}{(p+m)(p+m+1) \cdots(p+1)} \approx \\
& \frac{1}{\left(p+\frac{1}{2} m\right)^{m}}=\left(\frac{2}{h}\right)^{m} .
\end{aligned}
$$

To show Eq. (202), we use the power series representation of the Bessel functions, [23], 10.2.2, and an explicit result

$$
\begin{aligned}
& \int_{0}^{1} \rho^{a} R_{h}^{m}(\rho) \rho \mathrm{d} \rho= \\
& \quad \frac{(-1)^{p}(m-a)(m-a+2) \cdots(m-a+2 p-2)}{(m+a+2)(m+a+4) \cdots(m+a+2 p+2)},
\end{aligned}
$$

see [30], Appendix A, to write

$$
\begin{gathered}
I_{m h}^{-}=\int_{0}^{1} R_{m+2 p}^{m}(\rho) J_{m-2}(v \rho) \rho \mathrm{d} \rho=\left(\frac{1}{2} v\right)^{m-2} . \\
\sum_{k=0}^{\infty} \frac{\left(-\frac{1}{4} v^{2}\right)^{k}}{k !(k+m-2) !} \int_{0}^{1} \rho^{m+2 k-2} R_{m+2 p}^{m}(\rho) \rho \mathrm{d} \rho= \\
\frac{1}{2}\left(\frac{1}{2} v\right)^{m-2} \sum_{k=0}^{\infty} \frac{\left(-\frac{1}{4} v^{2}\right)^{k}}{k !(k+m-2) !} \cdot \\
\frac{(k-1)(k-2) \cdots(k-p)}{(k+m)(k+m+1) \cdots(k+m+p)} .
\end{gathered}
$$

In the last series in Eq. (206), the terms with $k=1,2, \cdots, p$ vanish, and setting the term with $k=0$ apart and rewriting the terms with $k=p+1, p+2, \cdots$, we get

$$
\begin{aligned}
I_{m h}^{-}= & \frac{1}{2}\left(\frac{1}{2} v\right)^{m-2}\left[\frac{(-1)^{p} p !(m-1)}{(m+p) !}\right. \\
& \left.+\sum_{k=p+1}^{\infty} \frac{\left(-\frac{1}{4} v^{2}\right)^{k}}{(k-p-1) !(k+m+p) !} \frac{k+m-1}{k}\right]
\end{aligned}
$$

As to the remaining series in Eq. (207), we shift the summation index $k$ by $p+1$ and recognize the power series of $J_{m+2 p+1}(v)$ to get

$$
\begin{gathered}
\frac{1}{2}\left(\frac{1}{2} v\right)^{m-2} \sum_{k=p+1}^{\infty} \frac{\left(-\frac{1}{4} v^{2}\right)^{k}}{(k-p-1) !(k+m+p) !} \frac{k+m-1}{k}= \\
(-1)^{p+1} \frac{J_{m+2 p+1}(v)}{v}+\frac{1}{2}(-1)^{p+1}\left(\frac{1}{2} v\right)^{m+2 p} \\
\sum_{k=0}^{\infty} \frac{\left(-\frac{1}{4} v^{2}\right)^{k}}{k !(k+m+2 p+1) !} \frac{m-1}{k+p+1}
\end{gathered}
$$

Finally, we use that

$$
\frac{1}{2(k+p+1)}=\int_{0}^{1}\left(w^{2}\right)^{k+p} w \mathrm{~d} w
$$

to write

$$
\begin{gathered}
\frac{1}{2}(-1)^{p+1}\left(\frac{1}{2} v\right)^{m+2 p} \sum_{k=0}^{\infty} \frac{\left(-\frac{1}{4} v^{2}\right)^{k}}{k !(k+m+2 p+1) !} \frac{m-1}{k+p+1}= \\
(-1)^{p+1}(m-1) \cdot \\
\int_{0}^{1}\left(\frac{1}{2} v\right)^{m+2 p} \sum_{k=0}^{\infty} \frac{\left(-\frac{1}{4} v^{2} w^{2}\right)^{k}}{k !(k+m+2 p+1) !} w^{2 p} w \mathrm{~d} w= \\
(-1)^{p+1}(m-1) \int_{0}^{1} \frac{2 J_{m+2 p+1}(v w)}{v} \frac{\mathrm{d} w}{w^{m}},
\end{gathered}
$$

where again the power series of $J_{m+2 p+1}(v w)$ has been used. This yields Eq. (202).

We finally consider

$$
I_{m h}^{+}=\int_{0}^{1} R_{h}^{m}(\rho) J_{m+2}(2 \pi r \rho) \rho d \rho
$$

for $m=0,1, \ldots$ and $h=m+2 p$ with $p=0,1, \ldots$. From

$$
\int_{0}^{1} J_{m+2}(2 \pi r \rho) R_{m+2+2 t}^{m+2}(\rho) \rho d \rho=(-1)^{t} \frac{J_{m+2 t+3}(2 \pi r)}{2 \pi r},
$$

for $t=0,1, \ldots$, we have

$$
\begin{aligned}
& J_{m+2}(2 \pi r \rho)= \\
& \quad \sum_{t=0}^{\infty} 2(m+2 t+3)(-1)^{t} \frac{J_{m+2 t+3}(2 \pi r)}{2 \pi r} R_{m+2+2 t}^{m+2}(\rho) .
\end{aligned}
$$

Therefore,

$$
I_{m h}^{+}=\sum_{t=0}^{\infty} 2(m+2 t+3)(-1)^{t} \frac{J_{m+2 t+3}(2 \pi r)}{2 \pi r} L_{m h t},
$$

where

$$
L_{m h t}=\int_{0}^{1} R_{m+2+2 t}^{m+2}(\rho) R_{h}^{m}(\rho) \rho d \rho
$$

for $t=0,1, \ldots$. Using Eq. (200) with $m+2$ instead of $m$, we have

$$
\begin{gathered}
R_{m+2+2 t}^{m+2}=\frac{t+1}{m+t+2} R_{m+2+2 t}^{m}+\frac{m+1}{(m+t+2)(t+1)} . \\
\sum_{k=1}^{t+1}(m+2 t-2 k+3) \frac{\left(\begin{array}{c}
t+1 \\
k
\end{array}\right)}{\left(\begin{array}{c}
m+t+1 \\
k
\end{array}\right)} . \\
(-1)^{k+1} R_{m+2 t-2 k+2}^{m} .
\end{gathered}
$$

Then by the orthogonality of the $R^{m}$,

$$
\int_{0}^{1} R_{n_{1}}^{m}(\rho) R_{n_{2}}^{m}(\rho) \rho d \rho=\frac{1}{2\left(n_{1,2}+1\right)} \delta_{n_{1} n_{2}},
$$

we get from Eq. (216) for $L_{m h t}$ in Eq. (215) with $h=m+2 p$

$$
L_{m h t}=0, \quad t=0,1, \ldots, p-2,
$$




$$
\begin{gathered}
L_{m h, p-1}=\frac{p}{2(m+p+1)(m+2 p+1)}, \\
L_{m h t}=\frac{m+1}{2(m+t+2)(t+1)} \frac{\left(\begin{array}{c}
t+1 \\
p
\end{array}\right)(-1)^{t-p}}{\left(\begin{array}{c}
m+t+1 \\
m+p
\end{array}\right)}, \\
t=p, p+1, \ldots
\end{gathered}
$$

Hence

$$
\begin{aligned}
I_{m, m+2 p}^{+}= & -(-1)^{p} \frac{p}{m+p+1} \frac{J_{m+2 p+1}(2 \pi r)}{2 \pi r} \\
+ & \\
& \left.\frac{(-1)^{p} \sum_{t=p}^{\infty} \frac{(m+1)(m+2 t+3)}{(t+1)(m+t+2)} .}{\left(\begin{array}{c}
m+t \\
p
\end{array}\right)} \begin{array}{l}
m+1 \\
m+p
\end{array}\right)
\end{aligned}
$$

Setting $t=s+p$ with $s=0,1, \ldots$ and using $h=m+2 p$, $q=m+p$, we obtain Eq. (120).

\section{References}

[1] F. Zernike, "Diffraction theory of the knife-edge test and its improved version, the phase-contrast method," Physica 1, 689-704 (1934).

[2] B. R. A. Nijboer, The Diffraction Theory of Aberrations (Ph.D. thesis, University of Groningen, The Netherlands, 1942).

[3] M. Born, and E. Wolf, Principles of Optics (Cambridge University Press, Cambridge, United Kingdom, 1999).

[4] A. J. E. M. Janssen, "Extended Nijboer-Zernike approach for the computation of optical point-spread functions," J. Opt. Soc. Am. A19, 849-857 (2002).

[5] J. J. M. Braat, P. Dirksen, and A. J. E. M. Janssen, “Assessment of an extended Nijboer-Zernike approach for the computation of optical point-spread functions," J. Opt. Soc. Am. A19, 858-870 (2002).

[6] P. Dirksen, J. J. M. Braat, P. De Bisschop, A. J. E. M. Janssen, C. A. H. Juffermans, and A. Williams, "Characterization of a projection lens using the extended Nijboer-Zernike approach," Proc. SPIE 4691, 1392-1399 (2002).

[7] P. Dirksen, J. J. M. Braat, A. J. E. M. Janssen, and C. Juffermans, "Aberration retrieval using the extended Nijboer-Zernike approach," J. Microlithogr. Microfabr. Microsyst. 2, 61-68 (2003).

[8] C. van der Avoort, J. J. M. Braat, P. Dirksen, and A. J. E. M. Janssen, "Aberration retrieval from the intensity point-spread function in the focal region using the extended Nijboer-Zernike approach," J. Mod. Opt. 52, 1695-1728 (2005).

[9] A. A. Ramos, and A. L. Ariste, "Image reconstruction with analytical point spread functions," Astron. Astrophys. 518, A6 (2010).

[10] X. Liu, L. Wang, J. Wang, and H. Meng, "A three-dimensional point spread function for phase retrieval and deconvolution," Opt. Express 14, 15392-15405 (2012).

[11] P. Riaud, D. Mawet, and A. Magette, “Nijboer-Zernike phase retrieval for high contrast imaging," Astron. Astrophys. 545, A150 (2012).
[12] J. J. M. Braat, P. Dirksen, A. J. E. M. Janssen, and A. S. van de Nes, "Extended Nijboer-Zernike representation of the vector field in the focal region of an aberrated high-aperture optical system," J. Opt. Soc. Am. 20, 2281-2292 (2003).

[13] V. S. Ignatowsky, "Diffraction by a lens of arbitrary aperture," Tr. Opt. Inst. 1, 1-36 (1919).

[14] B. Richards, and E. Wolf, "Electromagnetic diffraction in optical systems II. Structure of the image field in an aplanatic system," Proc. R. Soc. London Ser. A 253, 358-379 (1959).

[15] J. J. M. Braat, P. Dirksen, A. J. E. M. Janssen, S. van Haver, and A. S. van de Nes, "Extended Nijboer-Zernike approach to aberration and birefringence retrieval in a high-numericalaperture optical system," J. Opt. Soc. Am. A22, 2635-2650 (2005).

[16] S. van Haver, J. J. M. Braat, P. Dirksen, and A. J. E. M. Janssen, "High-NA aberration retrieval with the Extended Nijboer-Zernike vector diffraction theory," J. Europ. Opt. Soc. Rap. Public. 1, 06004 (2006).

[17] S. van Haver, J. J. M. Braat, A. J. E. M. Janssen, 0. T. A. Janssen, and S. F. Pereira, "Vectorial aerial-image computations of threedimensional objects based on the extended Nijboer-Zernike theory," J. Opt. Soc. Am. A26, 1221-1234 (2009).

[18] D. Flagello, T. Milster, and A. E. Rosenbluth, "Theory of high-NA imaging in homogeneous thin films," J. Opt. Soc. Am. A13, 53-64 (1996).

[19] J. J. M. Braat, S. van Haver, A. J. E. M. Janssen, and S. F. Pereira, "Image formation in a multilayer using the extended NijboerZernike theory," J. Europ. Opt. Soc. Rap. Public. 4, 09048 (2009).

[20] S. van Haver, The Extended Nijboer-Zernike Diffraction Theory and its Applications (Ph.D. thesis, Delft University of Technology, The Netherlands, 2010).

[21] J. J. M. Braat, S. van Haver, A. J. E. M. Janssen, and P. Dirksen, "Assessment of optical systems by means of point-spread functions," Progress in Optics, E. Wolf, ed., 51, 349-468 (Elsevier, Amsterdam, The Netherlands, 2008).

[22] 0. T. A. Janssen, S. van Haver, A. J. E. M. Janssen, J. J. M. Braat, H. P. Urbach, and S. F. Pereira, "Extended Nijboer-Zernike (ENZ) based mask imaging: efficient coupling of electromagnetic field solvers and the ENZ imaging algorithm," Proc. SPIE 6924, 692410 (2008).

[23] F. W. J. Olver, D. W. Lozier, R. F. Boisvert, and C. W. Clark, NIST Handbook of Mathematical Functions (Cambridge University Press, Cambridge, United Kingdom, 2010).

[24] E. C. Kintner, and R. M. Sillitto, "A new "analytic" method for computing the optical transfer function," Opt. Acta 23, 607-619 (1976).

[25] A. J. E. M. Janssen, J. J. M. Braat, and P. Dirksen, "On the computation of the Nijboer-Zernike aberration integrals at arbitrary defocus," J. Mod. Opt. 51, 687-703 (2004).

[26] J. Boersma, "On the computation of Lommel's functions of two variables," Math. Comput. 16, 232-238 (1962).

[27] R. M. Aarts, and A. J. E. M. Janssen, "On-axis and far-field sound radiation from resilient flat and dome-shaped radiators," J. Acoust. Soc. Am. 125, 1444-1455 (2009).

[28] W. J. Tango, "The circle polynomials of Zernike and their application in optics," Appl. Phys. 13, 327-332 (1977).

[29] F. G. Tricomi, Vorlesungen über Orthogonalreihen (Springer, Berlin, 1955). 
[30] J. J. M. Braat, and A. J. E. M. Janssen, “Double Zernike expansion of the optical aberration function from its power series expansion," J. Opt. Soc. Am. A30, 1213-1222 (2013). 